\title{
BUCKLING OF LONG COMPRESSION-LOADED ANISOTROPIC PLATES RESTRAINED AGAINST INPLANE LATERAL AND SHEAR DEFORMATIONS
}

\author{
Michael P. Nemeth* \\ Mechanics and Durability Branch, NASA Langley Research Center \\ Hampton, Virginia 23681-2199
}

\begin{abstract}
$\underline{\text { Abstract }}$
An approach for synthesizing buckling results and behavior for thin balanced and unbalanced symmetric laminates that are subjected to uniform axial compression loads and elastically restrained against inplane expansion, contraction, and shear deformation is presented. This approach uses a nondimensional analysis for infinitely long, flexurally anisotropic plates (coupling between bending and twisting) that are subjected to combined mechanical loads and is based on nondimensional parameters. In addition, nondimensional loading parameters are derived that account for the effects of the elastic inplane deformation restraints, membrane orthotropy, and membrane anisotropy on the induced prebuckling stress state. The loading parameters are used to determine buckling coefficients that include the effects of flexural orthotropy and flexural anisotropy. Many results are presented, for some selected laminates, that are intended to facilitate a structural designer's transition to the use of the generic buckling design curves that are presented and discussed in the paper. Several buckling response curves are presented that provide physical insight into the behavior for combined loads, in addition to providing useful design data. An example is presented that demonstrates the use of the generic design curves, which are applicable to a wide range of laminate constructions. The analysis approach and generic results indicate the effects and characteristics of laminate orthotropy and anisotropy in a very general and unifying manner.
\end{abstract}

\section{Introduction}

Structural tailoring of laminated-composite plates to enhance their buckling resistance is an important element in the development of new, advanced aerospace vehicles. One structural component that is often examined in design of aircraft and spacecraft is the long rectangular plate. Plates of this type commonly appear as elements of stiffened panels that are used for wing structures, and as semimonocoque shell segments that are used for fuselage and launch vehicle structures. Thus, establishing a broad understanding of the buckling behavior of long plates is es-

*Assistant Head. Associate Fellow, AIAA.

Copyright $(2003$ by the American Institute of Aeronautics and Astronautics, Inc. No copyright is asserted in the United States under Title 17, U. S. Code. The U. S. Government has a royaltyfree license to exercise all rights under the copyright claimed herein for Governmental purposes. All other rights are reserved by the copyright owner. sential to the advancement of structural-tailoring technology for aerospace vehicles.

An important type of long plate that appears as an element of advanced composite structures is the symmetrically laminated plate. In the present study, the term, "symmetrically laminated," refers to plates in which every lamina above the plate midplane has a corresponding lamina located at the same distance below the plate midplane, with the same thickness, material properties, and fiber orientation. Symmetrically laminated plates are, for the most part, flat after the manufacturing process and exhibit flat prebuckling deformation states, which is desirable for many applications. More importantly, the amenability of these plates to structural tailoring provides symmetrically laminated plates with a significant potential for reducing the weight of aerospace vehicles or for meeting special performance requirements. Thus, understanding the buckling behavior of symmetrically laminated plates is an important part of the search for ways to exploit plate orthotropy and anisotropy to reduce structural weight or to fulfill a special design requirement.

In many practical cases, symmetrically laminated plates exhibit specially orthotropic behavior. However, in many cases, these plates exhibit anisotropy in the form of material-induced coupling between pure bending and twisting deformations. This coupling is referred to herein as flexural anisotropy and it generally yields buckling modes that are skewed in appearance (see Fig. 1). Symmetrically laminated plates that are unbalanced are also being investigated for special-purpose uses in aerospace structures. In the present study, the term, "unbalanced laminate," refers to symmetric laminates in which each ply with a positive-valued fiber orientation is not "balanced" by a corresponding ply with a negative-valued fiber orientation. Unbalanced laminated plates exhibit anisotropy in the form of material-induced coupling between pure inplane dilatation and inplane shear deformations, in addition to flexural anisotropy. This coupling is referred to herein as membrane anisotropy and it generally yields combined inplane stress states for simple loadings like uniform edge compression when inplane displacement constraints are imposed on one or more edges of a plate. For example, when the two unloaded, opposite edges of an unbalanced, symmetrically laminated plate that is compression loaded, such as a $\left[+45_{2} / 0 /\right.$ 
90] laminate, are totally restrained against expansion and contraction and inplane shearing deformations, inplane shear stresses are developed in addition to the biaxial compression stresses that are typically present in balanced laminates (see Fig. 2). These kinematically induced shear stresses can be relatively large in magnitude, compared to the direct compressive stresses, and as a result can affect greatly the buckling behavior of the plate and yield buckling modes that are skewed in appearance.

The effects of flexural orthotropy and flexural anisotropy on the buckling behavior of long rectangular plates that are subjected to single and combined loading conditions are becoming better understood. For example, in-depth parametric studies that show the effects of flexural orthotropy and flexural anisotropy on the buckling behavior of long plates that are subjected to compression, shear, pure inplane bending, and various combinations of these loads have been presented in Refs. 1 through 3 . The results presented in these references detail the ways in which the importance of flexural anisotropy, on the buckling resistance of long plates, varies with the magnitude and type of the combined loading condition. Similar results for plates loaded by uniform shear and a general linear distribution of axial load across the plate width have also been presented in Ref. 4 .

Several studies have also addressed the behavior of rectangular plates that are restrained against inplane movement- an important research area because inplane movement is typically restricted in aerospace structures by adjacent panels and stiffeners. In particular, Harris ${ }^{5}$ examined the effects of lateral inplane restraint on the behavior of compression-loaded, specially orthotropic plates, and Obraztsov and Vasil' 'ev ${ }^{6}$ examined the same effects on compression-loaded, balanced angle-ply laminates. Sherbourne and Pandey ${ }^{7}$ examined the behavior of balanced and unbalanced, symmetric laminates subjected to uniaxial compression loads and with either lateral inplane movement restrained or both lateral movement and inplane shear deformation restrained. Their study highlights the effects of fiber orientation and plate aspect ratio for selected laminate stacking sequences made of a typical graphite-epoxy material system, and explored the possibility of tailoring laminates to have a negative Poisson's ratio, that results in improved buckling resistance (Insight into how laminate stacking sequence affects Poisson's ratio is found in Refs. 8-10.). Similar studies that focus on buckling-load optimization of compression-loaded rectangular laminates are presented in Refs. 11 and 12. These two studies examine the effects of unloaded edges that are either rigidly or elastically restrained agained inplane, lateral movement, and include the effects of transverse-shear flexibility. Results are presented for balanced, symmetric laminates made of a typical graphite-epoxy material, for several plate aspect ratios and five different boundary conditions. Bedair ${ }^{13,14}$ and Walker, et. al. ${ }^{15}$, have also presented studies that include the effects of restrained inplane movement, along with the presence of nonuniform applied compression loads. Specifically, the results in Refs. 13 and 14 focus on the behavior of finite-length isotropic plates with elastic inplane restraints. The results presented in Ref. 15 focus on buckling-load optimization of rectangular, graphite-epoxy laminates that are balanced and symmetric.

Studies that address the effects of membrane orthotropy and membrane anisotropy on the buckling behavior of long rectangular plates that are restrained against axial thermal expansion or contraction and subjected to uniform heating or cooling and mechanical loads have been presented in Refs. 16 and 17. Likewise, similar results for plates that are either fully restrained or elastically restrained against thermal expansion and contraction and subjected to uniform heating or cooling have been presented in Refs. 18 and 19, respectively. These studies are comprehensive and have provided a better understanding of the load interaction effects of balanced and unbalanced, symmetrically laminated plates that are subjected to mechanical loads and restrained against thermal expansion or contraction.

As evidenced by the previous studies discussed, the effects of membrane orthotropy and anisotropy, flexural orthotropy and anisotropy, and the restraint of inplane movement on the buckling behavior of rectangular plates that are subjected to mechanical and thermal loads are becoming better understood. However, comprehensive review of these studies indicates that there remains a need for in-depth studies that address, in a broad way, the effect of a compliant, elastic restraining medium on the buckling of compression-loaded, symmetrically laminated plates that are restrained against lateral expansion and contraction and inplane shearing deformations. This research area is important because it represents a class of problems that must be well understood in order to determine potential benefits and pitfalls of structural tailoring. Thus, the objective of the present study is to present an analytical approach that indicates the effects of a compliant, elastic restraining medium on the buckling behavior of compression-loaded, balanced and unbalanced, symmetrically laminated plates in a very general manner. Towards that objective, a buckling analysis is presented first that follows the analysis presented in Ref. 19. To achieve this objective, the buckling analysis is formulated in terms of nondimensional buckling coefficients and load factors that depend on the inplane compliance coefficients for a given plate and the relative compliance of a restraining medium. Results are then presented for infi- 
nitely long plates with the two long, unloaded edges clamped or simply supported and elastically restrained against inplane movement. These results include nondimenional buckling loads for selected laminates that are made from one of nine different material systems, and generic results that are applicable to a vast range of laminate constructions, including hybrid laminates. Buckling results for infinitely long plates are important because they often provide a practical estimate of the behavior of finite-length rectangular plates (lower bounds to festoon buckling curves), and they provide information that is useful in explaining the behavior of these finite-length plates. Moreover, knowledge of the behavior of infinitely long plates can provide insight into the buckling behavior of more complex structures such as stiffened panels. Finally, an example is presented that illustrates the use of the generic buckling-design curves presented herein and in Ref. 18, and highlights the effects of a restraining medium on the buckling behavior.

\section{Analysis Description}

In preparing generic design charts for buckling of a single flat thin plate, a special-purpose analysis is often preferred over a general-purpose analysis code, such as a finite-element code, because of the cost and effort that is usually involved in generating a large number of results with a general-purpose code. The results presented in the present paper were obtained by using such a special-purpose buckling analysis that is based on classical laminated-plate theory. The analysis details are lengthy; hence, only a brief description of the buckling analysis is presented herein. First, the buckling analysis for long plates that are subjected to a general set of mechanical loads is described. Then, the mechanical loads that are induced in compression-loaded plates by elastically restraining the inplane lateral and shear deformations are derived.

\section{Buckling Analysis}

Symmetrically laminated plates can have many different constructions because of the wide variety of material systems, fiber orientations, and stacking sequences that can be selected to construct a laminate. A way of coping with the large number of choices for laminate constructions is to use convenient nondimensional parameters in order to understand overall behavioral trends and sensitivities of the structural behavior to perturbations in laminate construction. The buckling analysis used in the present paper is based on classical laminatedplate theory and the classical Rayleigh-Ritz method, and is derived explicitly in terms of the nondimensional parameters defined in Refs. 1-4 and 16-20. This approach was motivated by the need for generic (independent of a specific laminate construction) parametric results for composite-plate buckling behavior that are expressed in terms of the minimum number of independent parameters needed to fully characterize the behavior, and that indicate the overall trends and sensitivity of the results to changes in the parameters. The nondimensional parameters that were used to formulate the buckling analysis are given by

$$
\begin{aligned}
& \alpha_{\infty}=\frac{b}{\lambda}\left(\frac{D_{11}}{D_{22}}\right)^{1 / 4} \\
& \beta=\frac{D_{12}+2 D_{66}}{\left(D_{11} D_{22}\right)^{1 / 2}} \\
& \gamma=\frac{D_{16}}{\left(D_{11}^{3} D_{22}\right)^{1 / 4}} \\
& \delta=\frac{D_{26}}{\left(D_{11} D_{22}^{3}\right)^{1 / 4}}
\end{aligned}
$$

where $b$ is the plate width and $\lambda$ is the half-wave length of the buckle pattern of an infinitely long plate (see Fig. 1). The subscripted D-terms are the bending stiffnesses of classical laminated-plate theory. The parameters $\alpha_{\infty}$ and $\beta$ characterize the flexural orthotropy, and the parameters $\gamma$ and $\delta$ characterize the flexural anisotropy.

The mechanical loading conditions that are included in the buckling analysis are uniform transverse tension or compression, uniform shear, and a general linear distribution of axial load across the plate width, as depicted in Fig. 1. Typically, an axial stress resultant distribution is partitioned into a uniform part and a pure bending part. However, this representation is not unique. The longitudinal stress resultant $\mathrm{N}_{\mathrm{x}}$ is partitioned in the analysis into a uniform tension or compression part and a linearly varying part that corresponds to eccentric inplane bending loads. This partitioning is given by

$$
\mathrm{N}_{\mathrm{x}}=\mathrm{N}_{\mathrm{xc}}-\mathrm{N}_{\mathrm{b}}\left[\varepsilon_{0}+\left(\varepsilon_{1}-\varepsilon_{0}\right) \eta\right]
$$

where $\mathrm{N}_{\mathrm{xc}}$ denotes the intensity of the constant-valued tension or compression part of the load, and the term containing $\mathrm{N}_{\mathrm{b}}$ defines the intensity of the eccentric inplane bending load distribution. The symbols $\varepsilon_{0}$ and $\varepsilon_{1}$ define the distribution of the inplane bending load, and the symbol $\eta$ is the nondimensional coordinate given by $\eta=y / b$. This particular way of partitioning the longitudinal stress resultant was used for convenience by eliminating the need to calculate the uniform and pure bending parts of an axial stress resultant distribution prior to performing a buckling analysis.

The analysis is based on a general formulation that includes combined destabilizing loads that are proportional to a positive-valued loading parameter $\widetilde{p}$ that is increased until buckling occurs, and independent subcrit- 
ical combined loads that remain fixed at a specified load level below the value of the buckling load. Herein, the term "subcritical load" is defined as any load that does not cause buckling to occur. In practice, the subcritical loads are applied to a plate prior to, and independent of, the destabilizing loads with an intensity below that which will cause the plate to buckle. Then, with the subcritical loads fixed, the active, destabilizing loads are applied by increasing the magnitude of the loading parameter until buckling occurs. This approach permits certain types of combined-load interaction to be investigated in a direct and convenient manner. For example, in analyzing the stability of an aircraft fuselage, the nondestabilizing transverse tension load in a fuselage panel that is caused by cabin pressurization can be considered to remain constant and, as a result, it can be represented as a passive, subcritical load. The combined shear, compression, and inplane bending loads that are caused by flight maneuvers can vary and cause buckling and, as a result, they can be represented as active, destabilizing loads.

The distinction between the active, destabilizing and passive subcritical loading systems is implemented in the buckling analysis by partitioning the prebuckling stress resultants as follows

$$
\begin{aligned}
& N_{x c}=-N_{x 1}^{c}+N_{x 2}^{c} \\
& N_{y}=-N_{y 1}+N_{y 2} \\
& N_{x y}=N_{x y 1}+N_{x y 2} \\
& N_{b}=N_{b 1}+N_{b 2}
\end{aligned}
$$

where the stress resultants with the subscript 1 are the destabilizing loads, and those with the subscript 2 are the subcritical loads. The sign convention used herein for positive values of these stress resultants is shown in Fig. 1. In particular, positive values of the general linear edge stress distribution parameters $\mathrm{N}_{\mathrm{b} 1}, \mathrm{~N}_{\mathrm{b} 2}, \varepsilon_{0}$, and $\varepsilon_{1}$ correspond to compression loads. Negative values of $\mathrm{N}_{\mathrm{b} 1}$ and $\mathrm{N}_{\mathrm{b} 2}$, or negative values of either $\varepsilon_{0}$ or $\varepsilon_{1}$, yield linearly varying stress distributions that include tension. Depictions of a variety of inplane bending load distributions are given in Ref. 4. The two normal-stress resultants of the system of destabilizing loads, $\mathrm{N}_{\mathrm{x} 1}^{\mathrm{c}}$ and $\mathrm{N}_{\mathrm{y} 1}$, are defined to be positive-valued for compression loads. This convention results in positive eigenvalues being used to indicate instability due to uniform compression loads.

The buckling analysis includes several nondimensional stress resultants associated with Eqs. (6) through (9). These dimensionless stress resultants are given by

$$
\mathrm{n}_{\mathrm{xj}}^{\mathrm{c}}=\frac{\mathrm{N}_{\mathrm{xj}}^{\mathrm{c}} \mathrm{b}^{2}}{\pi^{2}\left(\mathrm{D}_{11} \mathrm{D}_{22}\right)^{1 / 2}}
$$

$$
\begin{aligned}
& \mathrm{n}_{\mathrm{yj}}=\frac{\mathrm{N}_{\mathrm{yj}} \mathrm{b}^{2}}{\pi^{2} \mathrm{D}_{22}} \\
& \mathrm{n}_{\mathrm{xyj}}=\frac{\mathrm{N}_{\mathrm{xyj}} b^{2}}{\pi^{2}\left(\mathrm{D}_{11} D_{22}^{3}\right)^{1 / 4}} \\
& \mathrm{n}_{\mathrm{bj}}=\frac{\mathrm{N}_{\mathrm{bj}} b^{2}}{\pi^{2}\left(\mathrm{D}_{11} D_{22}\right)^{1 / 2}}
\end{aligned}
$$

where the subscript $\mathrm{j}$ takes on the values of 1 and 2. In addition, the destabilizing loads are expressed in terms of the loading parameter $\widetilde{p}$ in the analysis by

$$
\begin{aligned}
& \mathrm{n}_{\mathrm{x} 1}^{\mathrm{c}}=\mathrm{L}_{1} \widetilde{\mathrm{p}} \\
& \mathrm{n}_{\mathrm{y} 1}=\mathrm{L}_{2} \widetilde{\mathrm{p}} \\
& \mathrm{n}_{\mathrm{xy} 1}=\mathrm{L}_{3} \widetilde{\mathrm{p}} \\
& \mathrm{n}_{\mathrm{b} 1}=\mathrm{L}_{4} \widetilde{\mathrm{p}}
\end{aligned}
$$

where $\mathrm{L}_{1}$ through $\mathrm{L}_{4}$ are load factors that determine the specific form (relative contributions of the load components) of a given system of destabilizing loads. Typically, the dominant load factor is assigned a value of 1 and all others are given as positive or negative fractions.

Nondimensional buckling coefficients that are used herein are given by the values of the dimensionless stress resultants of the system of destabilizing loads at the onset of buckling; i.e.,

$$
\begin{aligned}
& \mathrm{K}_{\mathrm{x}} \equiv\left(\mathrm{n}_{\mathrm{x} 1}^{\mathrm{c}}\right)_{\mathrm{cr}}=\frac{\left(\mathrm{N}_{\mathrm{x} 1}^{\mathrm{c}}\right)_{\mathrm{cr}} \mathrm{b}^{2}}{\pi^{2}\left(\mathrm{D}_{11} \mathrm{D}_{22}\right)^{1 / 2}}=\mathrm{L}_{1} \widetilde{\mathrm{p}}_{\mathrm{cr}} \\
& \mathrm{K}_{\mathrm{y}} \equiv\left(\mathrm{n}_{\mathrm{y} 1}\right)_{\mathrm{cr}}=\frac{\left(\mathrm{N}_{\mathrm{y} 1}\right)_{\mathrm{cr}} \mathrm{b}^{2}}{\pi^{2} \mathrm{D}_{22}}=\mathrm{L}_{2} \widetilde{\mathrm{p}}_{\mathrm{cr}} \\
& \mathrm{K}_{\mathrm{s}} \equiv\left(\mathrm{n}_{\mathrm{xy} 1}\right)_{\mathrm{cr}}=\frac{\left(\mathrm{N}_{\mathrm{xy} 1}\right)_{\mathrm{cr}} \mathrm{b}^{2}}{\pi^{2}\left(\mathrm{D}_{11} \mathrm{D}_{22}^{3}\right)^{1 / 4}}=\mathrm{L}_{3} \widetilde{\mathrm{p}}_{\mathrm{cr}} \\
& \mathrm{K}_{\mathrm{b}} \equiv\left(\mathrm{n}_{\mathrm{b} 1}\right)_{\mathrm{cr}}=\frac{\left(\mathrm{N}_{\mathrm{b} 1}\right)_{\mathrm{cr}} \mathrm{b}^{2}}{\pi^{2}\left(\mathrm{D}_{11} \mathrm{D}_{22}\right)^{1 / 2}}=\mathrm{L}_{4} \widetilde{\mathrm{p}}_{\mathrm{cr}}
\end{aligned}
$$

where the quantities enclosed in the parentheses with the subscript "cr" are critical values that correspond to buckling and $\widetilde{\mathrm{p}}_{\mathrm{cr}}$ is the magnitude of the loading parameter at buckling. Positive values of the coefficients $\mathrm{K}_{\mathrm{x}}$ and $\mathrm{K}_{\mathrm{y}}$ correspond to uniform compression loads, and the coefficient $\mathrm{K}_{\mathrm{s}}$ corresponds to uniform positive shear. The direction of a positive shear-stress resultant that acts on a plate is shown in Fig. 1. The coefficient $\mathrm{K}_{\mathrm{b}}$ corresponds to the specific inplane bending load distribution defined by the selected values of the parameters $\varepsilon_{0}$ and $\varepsilon_{1}$ (see Fig. 1). 
The mathematical expression used in the variational analysis to represent the general off-center and skewed buckle pattern is given by

$$
\mathrm{w}_{\mathrm{N}}(\xi, \eta)=\sum_{\mathrm{m}=1}^{\mathrm{N}}\left(\mathrm{A}_{\mathrm{m}} \sin \pi \xi+\mathrm{B}_{\mathrm{m}} \cos \pi \xi\right) \Phi_{\mathrm{m}}(\eta)
$$

where $\xi=x / \lambda$ and $\eta=y / b$ are nondimensional coordinates, $\mathrm{w}_{\mathrm{N}}$ is the out-of-plane displacement field, and $A_{m}$ and $B_{m}$ are the unknown displacement amplitudes. In accordance with the Rayleigh-Ritz method, the basis functions $\Phi_{\mathrm{m}}(\eta)$ are required to satisfy the kinematic boundary conditions on the plate edges at $\eta=$ 0 and 1. For the simply supported plates, the basis functions used in the analysis are given by

$$
\Phi_{\mathrm{m}}(\eta)=\sin \mathrm{m} \pi \eta
$$

for values of $m=1,2,3, \ldots, N$. Similarly, for the clamped plates, the basis functions are given by

$$
\Phi_{m}(\eta)=\cos (m-1) \pi \eta-\cos (m+1) \pi \eta
$$

For both boundary conditions, the two long edges of a plate are free to move in-plane, unless noted otherwise.

Algebraic equations that govern the buckling behavior of infinitely long plates are obtained by substituting the series expansion for the buckling mode given by Eq. (22) into a nondimensionalized form of the second variation of the total potential energy and then computing the integrals appearing in the nondimensional second variation in closed form. The resulting equations constitute a generalized eigenvalue problem that depends on the aspect ratio of the buckle pattern $\lambda / b$ (see Fig. 1) and the nondimensional parameters and nondimensional stress resultants defined herein. The smallest eigenvalue of the problem corresponds to buckling and is found by specifying a value of $\lambda / b$ and solving the corresponding generalized eigenvalue problem for its smallest eigenvalue. This process is repeated for successive values of

$\lambda / b$ until the overall smallest eigenvalue is found.

Results that were obtained from the analysis described herein for uniform compression, uniform shear, pure inplane bending (given by $\varepsilon_{0}=-1$ and $\varepsilon_{1}=1$ ), and various combinations of these mechanical loads have been compared with other results for isotropic, orthotropic, and anisotropic plates that were obtained by using other analysis methods. These comparisons are discussed in Refs. 1-3, and in every case the results described herein were found to be in good agreement with those obtained from other analyses. Likewise, results were obtained for isotropic and specially orthotropic plates that are subjected to a general linear distribution of axial load across the plate width and compared with results that were obtained by seven different authors (see Ref. 4). In every case, the agreement was good.

\section{Prebuckling Stress Resultants}

In general, compression-loaded plates that are symmetrically laminated, but unbalanced, become subjected to a combined inplane stress state when the lateral, inplane expansion and contraction and inplane shearing deformations are restrained at the plate edges (see Fig. 2). As the magnitude of the compression load increases, the induced loads increase proportionally, which can cause premature buckling, compared to the buckling resistance of the corresponding unrestrained plate. These induced mechanical loads are determined in the present study by using the inverted membrane constitutive equations that are based on classical laminated-plate theory; that is,

$$
\left\{\begin{array}{c}
\varepsilon_{\mathrm{x}} \\
\varepsilon_{\mathrm{y}} \\
\gamma_{\mathrm{xy}}
\end{array}\right\}=\left[\begin{array}{lll}
\mathrm{a}_{11} & \mathrm{a}_{12} & \mathrm{a}_{16} \\
\mathrm{a}_{12} & \mathrm{a}_{22} & \mathrm{a}_{26} \\
\mathrm{a}_{16} & \mathrm{a}_{26} & \mathrm{a}_{66}
\end{array}\right]\left\{\begin{array}{c}
\mathrm{N}_{\mathrm{x}} \\
\mathrm{N}_{\mathrm{y}} \\
\mathrm{N}_{\mathrm{xy}}
\end{array}\right\}
$$

where $\varepsilon_{x}, \varepsilon_{y}$, and $\gamma_{x y}$ are the prebuckling, inplane strains and the subscripted a-terms are the plate membrane compliance coefficients. An alternate form of this equation (see Ref. 21, p. 79) that is also used in the present study, that utilizes the overall laminate properties, is given by

$$
\left\{\begin{array}{c}
\varepsilon_{x} \\
\varepsilon_{y} \\
\gamma_{x y}
\end{array}\right\}=\left[\begin{array}{ccc}
\frac{1}{E_{x}} & -\frac{v_{y x}}{E_{y}} & \frac{\eta_{x, x y}}{G_{x y}} \\
-\frac{v_{x y}}{E_{x}} & \frac{1}{E_{y}} & \frac{\eta_{y, x y}}{G_{x y}} \\
\frac{\eta_{x y, x}}{E_{x}} & \frac{\eta_{x y, y}}{E_{y}} & \frac{1}{G_{x y}}
\end{array}\right]\left\{\begin{array}{c}
N_{x} / h \\
N_{y} / h \\
N_{x y} / h
\end{array}\right\}
$$

where $\mathrm{h}$ is the laminate thickness, $\mathrm{E}_{\mathrm{x}}$ and $\mathrm{E}_{\mathrm{y}}$ are the laminate moduli, $\mathrm{G}_{\mathrm{xy}}$ is the laminate shear moduli, $v_{\mathrm{xy}}$ and $v_{\mathrm{yx}}$ are the major and minor Poisson's ratios, respectively, $\eta_{x, x y}$ and $\eta_{y, x y}$ are the coefficients of mutual influence of the first kind, and $\eta_{x y, x}$ and $\eta_{x y, y}$ are the coefficients of mutual influence of the second kind. Relationships between the various constitutive terms are obtained by noting that the coefficient matrix of Eq. (25b) is symmetric. Following Ref. 21, the coefficients of mutual influence are referred to herein as shearextension coupling coefficients.

The effects of the elastic boundary restraints depicted in Fig. 2a are obtained by noting that the induced stress resultants are proportional to the strains caused by 
expansions or contractions and shearing deformations of the plate and the resistance provided by the restraining medium. Typically, the elastic resistance of the restraining medium is simulated with linear springs and expressed in terms of the corresponding spring stiffnesses. In the present study, the elastic resistance of a homogeneous restraining medium is described approximately in terms of two overall compliance coefficients of the restraining medium, denoted by $\mathrm{C}_{2}$ and $\mathrm{C}_{3}$. In particular, the action of the elastic restraining medium is represented in a simple manner by

$$
\varepsilon_{\mathrm{y}}=-\mathrm{C}_{2} \mathrm{~N}_{\mathrm{y}}
$$

and

$$
\gamma_{\mathrm{xy}}=-\mathrm{C}_{3} \mathrm{~N}_{\mathrm{xy}}
$$

for a uniform, positive-valued set of strains in a plate. The negative sign in Eq. (26) indicates that a positive, expansional strain in the $y$-direction is reacted by a compressive stress in the $y$-direction, that corresponds to a negative value for $\mathrm{N}_{\mathrm{y}}$. In other words, for a plate that is free to deform under axial loading, a restraining medium would require a compressive restoring force to suppress the deformation. Similarly, the negative sign in Eq. (27) indicates that a positive shearing strain induces, or is reacted by, a negative shearing-stress resultant. In the present study, the plates are presumed to be supported and loaded such that nonuniformities in the prebuckling stress field are negligible.

Expressions for the induced stress resultants are obtained by substituting Eqs. (26) and (27) into Eq. (25a) and then solving two of the resulting equations for $\mathrm{N}_{\mathrm{y}}$ and $\mathrm{N}_{\mathrm{xy}}$ in terms of the applied stress resultant $\mathrm{N}_{\mathrm{x}}$. This step gives

$$
N_{y}=N_{x} \frac{a_{16} a_{26}-a_{12}\left(a_{66}+C_{3}\right)}{\left(a_{22}+C_{2}\right)\left(a_{66}+C_{3}\right)-a_{26}^{2}}
$$

and

$$
\mathrm{N}_{\mathrm{xy}}=\mathrm{N}_{\mathrm{x}} \frac{\mathrm{a}_{12} \mathrm{a}_{26}-\mathrm{a}_{16}\left(\mathrm{a}_{22}+\mathrm{C}_{2}\right)}{\left(\mathrm{a}_{22}+\mathrm{C}_{2}\right)\left(\mathrm{a}_{66}+\mathrm{C}_{3}\right)-\mathrm{a}_{26}^{2}}
$$

To simplify these two equations further and to provide a simple way to estimate the influence of a restraining medium, the compliance coefficients of the restraining medium are defined as relative proportions of the plate compliance coefficients; that is,

$$
\mathrm{C}_{2}=\mathrm{R}_{2} \mathrm{a}_{22}
$$

and

$$
\mathrm{C}_{3}=\mathrm{R}_{3} \mathrm{a}_{66}
$$

Because of these definitions, $R_{2}$ and $R_{3}$ are referred to herein as compliance ratios. By using Eqs. (30) and (31), Eqs. (28) and (29) become

$$
N_{y}=N_{x} \frac{a_{16} a_{26}-a_{12} a_{66}\left(1+R_{3}\right)}{a_{22} a_{66}\left(1+R_{2}\right)\left(1+R_{3}\right)-a_{26}^{2}}
$$

and

$$
N_{x y}=N_{x} \frac{a_{12} a_{26}-a_{16} a_{22}\left(1+R_{2}\right)}{a_{22} a_{66}\left(1+R_{2}\right)\left(1+R_{3}\right)-a_{26}^{2}}
$$

Next, because all the subcritical loads used in the buckling analysis are zero-valued for this problem, $\mathrm{N}_{\mathrm{x}}=$ $\mathrm{N}_{\mathrm{x} 1}^{\mathrm{c}}, \mathrm{N}_{\mathrm{y}}=-\mathrm{N}_{\mathrm{y} 1}$, and $\mathrm{N}_{\mathrm{xy}}=\mathrm{N}_{\mathrm{xy} 1}$ (see Figs. 1 and 2). This substitution yields

$$
\mathrm{N}_{\mathrm{y} 1}=\mathrm{N}_{\mathrm{x} 1}^{\mathrm{c}} \frac{\mathrm{a}_{16} \mathrm{a}_{26}-\mathrm{a}_{12} \mathrm{a}_{66}\left(1+\mathrm{R}_{3}\right)}{\mathrm{a}_{22} \mathrm{a}_{66}\left(1+\mathrm{R}_{2}\right)\left(1+\mathrm{R}_{3}\right)-\mathrm{a}_{26}^{2}}
$$

and

$$
\mathrm{N}_{\mathrm{xy} 1}=-\mathrm{N}_{\mathrm{x} 1}^{\mathrm{c}} \frac{\mathrm{a}_{12} \mathrm{a}_{26}-\mathrm{a}_{16} \mathrm{a}_{22}\left(1+\mathrm{R}_{2}\right)}{\mathrm{a}_{22} \mathrm{a}_{66}\left(1+\mathrm{R}_{2}\right)\left(1+\mathrm{R}_{3}\right)-\mathrm{a}_{26}^{2}}
$$

Equations similar to equations (34) and (35), that express the induced stress resultants in terms of the overall laminate material properties, are obtained by substituting Eqs. (26), (27), (30), and (31) into Eq. (25b) and then solving two of the resulting equations for $\mathrm{N}_{\mathrm{y}}$ and $\mathrm{N}_{\mathrm{xy}}$ in terms of the applied stress resultant $\mathrm{N}_{\mathrm{x}}$. This procedure gives

$$
N_{y 1}=N_{x 1}^{c} \frac{\left(1+R_{3}\right) v_{y x}+\eta_{x y, y} \eta_{x, x y}}{\left(1+R_{2}\right)\left(1+R_{3}\right)-\eta_{x y, y} \eta_{y, x y}}
$$

and

$$
N_{x y 1}=N_{x 1}^{c} \frac{\left(1+R_{2}\right) \eta_{x, x y}+v_{y x} \eta_{y, x y}}{\left(1+R_{2}\right)\left(1+R_{3}\right)-\eta_{x y, y} \eta_{y, x y}}
$$

Although the focus of the present study in on compression loaded plates $\left(\mathrm{N}_{\mathrm{x} 1}^{\mathrm{c}}>0\right)$, Eqs. (34) through (37) clearly indicate that buckling can occur under axial tension loads for some laminate constructions.

Equations (34) through (37) contain three special cases of interest. First, when a plate is rigidly restrained such that $\varepsilon_{y}=\gamma_{x y}=0$, the compliance ratios $R_{2}$ and $R_{3}$ are zero valued. For this case, Eqs. (34) through (37) give

$$
\frac{N_{y 1}}{N_{x 1}^{c}}=\frac{A_{12}}{A_{11}}=\frac{v_{y x}+\eta_{x y, y} \eta_{x, x y}}{1-\eta_{x y, y} \eta_{y, x y}}
$$


and

$$
\frac{N_{x y 1}}{N_{x 1}^{c}}=-\frac{A_{16}}{A_{11}}=\frac{\eta_{x, x y}+v_{y x} \eta_{y, x y}}{1-\eta_{x y, y} \eta_{y, x y}}
$$

where $A_{11}, A_{12}$, and $A_{16}$ are membrane stiffnesses of classical laminated-plate theory. Second, when a plate is elastically restrained against lateral expansion or contraction and unrestrained against inplane shearing deformations such that $\mathrm{N}_{\mathrm{xy} 1}=0$, the compliance ratio $\mathrm{R}_{3}$ $\rightarrow \infty$. This value for $R_{3}$ means that the restraining medium is much more compliant in shear than the plate.

For this case, Eqs. (34) through (37) give $\mathrm{N}_{\mathrm{xy} 1}=0$ and

$$
\frac{\mathrm{N}_{\mathrm{y} 1}}{\mathrm{~N}_{\mathrm{x} 1}^{\mathrm{c}}}=\frac{v_{\mathrm{yx}}}{\left(1+\mathrm{R}_{2}\right)}
$$

where

$$
v_{y x}=-\frac{a_{12}}{a_{22}}=\frac{A_{12} A_{66}-A_{16} A_{26}}{A_{11} A_{66}-A_{16}^{2}}
$$

Equation (40) agrees with the result given in Ref. 7 for the even simpler case when $R_{2}=0$. Third, when a plate is elastically restrained against inplane shearing deformations and unrestrained against lateral expansion or contraction such that $\mathrm{N}_{\mathrm{y} 1}=0$, the compliance ratio $\mathrm{R}_{2}$ $\rightarrow \infty$. This value for $R_{2}$ means that the restraining medium is much more compliant in lateral expansion than the plate. For this case, Eqs. (34) through (37) give $\mathrm{N}_{\mathrm{y} 1}=0$ and

$$
\frac{N_{x y 1}}{N_{x 1}^{c}}=\frac{\eta_{x, x y}}{\left(1+R_{3}\right)}
$$

where

$$
\eta_{x, x y}=\frac{a_{16}}{a_{66}}=\frac{A_{12} A_{26}-A_{16} A_{22}}{A_{11} A_{22}-A_{12}^{2}}
$$

Equations (34) through (37) define a combined loading state that is induced by elastically restraining the inplane lateral and shearing deformations of a plate. The buckling problem is posed by determining the load factors $\mathrm{L}_{2}$ and $\mathrm{L}_{3}$ that appear in Eqs. (15) and (16). For an applied compression load $\mathrm{N}_{\mathrm{x} 1}^{\mathrm{c}}$, the load factor $\mathrm{L}_{1}=1$ by definition. The values for the other two load factors that are needed to completely define the prebuckling stress state in the nondimensional buckling analysis are obtained by dividing Eqs. (15) and (16) by Eq. (14), with $\mathrm{L}_{1}$ $=1$, and by using Eqs. (10) - (12). This step yields

$$
\begin{aligned}
L_{2}= & \frac{N_{y 1}}{N_{x 1}^{c}}\left(\frac{D_{11}}{D_{22}}\right)^{1 / 2}= \\
L_{3}= & \frac{N_{x y 1}}{N_{x 1}^{c}}\left(\frac{a_{16} a_{26}-a_{12} a_{66}\left(1+R_{3}\right)}{a_{22} a_{66}}\right)^{1 / 4}= \\
& -\left[\frac{\left.a_{12}\right)\left(1+R_{3}\right)-a_{26}^{2}}{a_{22} a_{66}\left(1+R_{26}\right)\left(1+R_{3}\right)-a_{26}^{2}}\right]\left(\frac{D_{11}}{D_{22}}\right)^{1 / 2}
\end{aligned}
$$

For balanced laminates, $a_{16}=a_{26}=0$, Eq. (45) gives $L_{3}=$ 0, and Eq. (44) becomes

$$
L_{2}=\frac{N_{y 1}}{N_{x 1}^{c}}\left(\frac{D_{11}}{D_{22}}\right)^{1 / 2}=\left[\frac{v_{y x}}{\left(1+R_{2}\right)}\right]\left(\frac{D_{11}}{D_{22}}\right)^{1 / 2}
$$

where $v_{y x}=\frac{A_{12}}{A_{11}}$. For an isotropic plate, Eq. (46) reduces to

$$
\mathrm{L}_{2}=\frac{\mathrm{N}_{\mathrm{y} 1}}{\mathrm{~N}_{\mathrm{x} 1}^{\mathrm{c}}}=\frac{\mathrm{v}}{\left(1+\mathrm{R}_{2}\right)}
$$

where $v$ is Poisson's ratio of a homogeneous, isotropic material. With $\mathrm{L}_{1}=1$ and $\mathrm{L}_{2}$ and $\mathrm{L}_{3}$ defined by Eqs. (44) and (45), the critical value of the mechanical loading parameter $\widetilde{\mathrm{p}}_{\mathrm{cr}}$ can be calculated by using the nondimensional buckling analysis. Note that $\widetilde{\mathrm{p}}_{\mathrm{cr}}=$ $\widetilde{\mathrm{p}}_{\mathrm{cr}}\left(\beta, \gamma, \delta, \mathrm{L}_{2}, \mathrm{~L}_{3}\right)$ for a given set of bending boundary conditions (e.g., simply supported and clamped edges).

Finally, it is important to mention that the approach used herein to define the prebuckling stress state also applies for a more sophisticated plate theory, like a first-order transverse-shear deformation theory, because the inplane stiffness and compliance coefficients are identical to those of classical laminated-plate theory. For this theory, $\widetilde{\mathrm{p}}_{\mathrm{cr}}$ would depend also upon additional nondimensional parameters that characterize the transverseshear flexibility. Thus, the only difference in the results for the two plate bending theories is the actual value of $\widetilde{\mathrm{p}}_{\text {cr }}$ that is used in Eqs. (18)-(20), for a given problem. It is also important to point out that $\widetilde{\mathrm{p}}_{\mathrm{cr}}$ for a long plate does not depend on the buckle aspect ratio parameter $\alpha_{\infty}$. This fact has been shown and discussed in Refs. 1-4. 


\section{Results for Selected Laminates}

Results are presented in this section that illustrate the behavioral trends for several selected symmetrically laminated plates that are loaded by uniform axial compression. Nine different material systems are considered that include boron-aluminum, S-glass-epoxy, a typical boron-epoxy, AS4/3501-6 graphite-epoxy, AS4/3502 graphite-epoxy, IM7/5260 graphite-bismaleimide, Kevlar 49-epoxy, IM7/PETI-5, and P-100/3502 pitch-epoxy materials (see Table 1). The plates are either rigidly restrained, elastically restrained against only lateral, inplane movement, elastically restrained against only inplane shear deformation, or are elastically restrained against both lateral inplane movement and inplane shearing deformations. First, results are presented in Table 2 and Figs. 3-10 for several balanced, symmetric laminates that exhibit relatively small degrees of flexural anisotropy; that is, $\left[\left( \pm 45 / 0_{2}\right)_{\mathrm{m}}\right]_{\mathrm{s}}$ axially stiff laminates, $[( \pm 45 /$ $\left.\left.90_{2}\right)_{\mathrm{m}}\right]_{\mathrm{s}}$ laterally stiff laminates, $\left[( \pm 45 / 0 / 90)_{\mathrm{m}}\right]_{\mathrm{s}}$ quasi-isotropic laminates, and $\left[( \pm \theta)_{\mathrm{m}}\right]_{\mathrm{s}}$ angle-ply laminates. Then, results are presented for $\left[\left(+45_{2} / 0_{2}\right)_{\mathrm{m}}\right]_{\mathrm{s}},\left[\left(+45_{2} / 90_{2}\right)_{\mathrm{m}}\right]_{\mathrm{s}}$, and $\left[\left(+45_{2} / 0 / 90\right)_{\mathrm{m}}\right]_{\mathrm{s}}$ unbalanced laminates in Tables 3-4 and Figs. 11-21 that exhibit significant degrees of membrane and flexural anisotropies. In addition, results are presented in Figs. 22-26 for $\left[+\theta_{3} / 15\right]_{\mathrm{s}}$ unbalanced laminates that exhibit a wide range of values for the overall laminate Poisson's ratio, including negative values. All the results are based on classical laminated-plate theory and the nominal ply thickness used in the calculations was 0.005 in.

\section{$\underline{\text { Results for Balanced Laminates }}$}

For balanced laminates, there are no inplane shearing deformations because $\mathrm{A}_{16}=\mathrm{A}_{26}=\mathrm{a}_{16}=\mathrm{a}_{26}=0$, and as a result, the compliance ratio $R_{3}$ is immaterial and the induced shearing stress resultant $\mathrm{N}_{\mathrm{xy} 1}$ is zero valued. With the use of Eq. (41), Eq. (36) simplifies to

$$
\left(1+R_{2}\right) \frac{N_{y 1}}{N_{x 1}^{c}}=v_{y x}=\frac{A_{12}}{A_{11}}
$$

where $0 \leq R_{2}<\infty$. Thus, graphs or tables of $v_{y x}$ for balanced, symmetric laminates yield the induced lateral stress resultant $\mathrm{N}_{\mathrm{y} 1}$ for an infinite number of different compliance ratios.

Results are presented in Table 2 that show the load ratio $\left(1+R_{2}\right) \frac{N_{y 1}}{N_{x 1}^{c}}$, or equivalently, $v_{y x}$ for $\left[\left( \pm 45 / 0_{2}\right)_{m}\right]_{s}$, $\left[\left( \pm 45 / 90_{2}\right)_{\mathrm{m}}\right]_{\mathrm{s}}$, and $\left[( \pm 45 / 0 / 90)_{\mathrm{m}}\right]_{\mathrm{s}}$ laminates made from one of the nine different material systems given in Table 1. The results are independent of the number of laminate plies and indicate that the laterally stiff $\left[\left( \pm 45 / 90_{2}\right)_{\mathrm{m}}\right]_{\mathrm{s}}$ laminates exhibit the largest values of $v_{\mathrm{yx}}$ for a given material system; the smallest values are exhibited by the axially stiff $\left[\left( \pm 45 / 0_{2}\right)_{\mathrm{m}}\right]_{\mathrm{s}}$ laminates. Moreover, for the $\left[\left( \pm 45 / 90_{2}\right)_{\mathrm{m}}\right]_{\mathrm{s}}$ and $\left[\left( \pm 45 / 0_{2}\right)_{\mathrm{m}}\right]_{\mathrm{s}}$ laminates, the largest value of $v_{y x}$ is obtained for the P-100/3502 pitch-epoxy and the boron-aluminum materials, respectively. For the $\left[( \pm 45 / 0 / 90)_{\mathrm{m}}\right]_{\mathrm{s}}$ quasi-isotropic laminates, the largest value of $v_{y x}$ is obtained for the Kevlar 49-epoxy material. For all the laminates and material systems, $0<\mathrm{N}_{\mathrm{y} 1}<\mathrm{N}_{\mathrm{x} 1}^{\mathrm{c}}$ for all allowable values of the compliance ratio $R_{2}$ and the laminates experience a state of uniform biaxial compression prior to buckling.

Nondimensional buckling loads are shown in Fig. 3 as a function of the number of laminate plies for the axially stiff $\left[\left( \pm 45 / 0_{2}\right)_{\mathrm{m}}\right]_{\mathrm{s}}$ laminates made of the IM7/5260 material. The nondimensional buckling load is given by

$$
\frac{\left(\mathrm{N}_{\mathrm{x} 1}^{\mathrm{c}}\right)_{\mathrm{cr}} \mathrm{b}^{2}}{\pi^{2} \overline{\mathrm{D}}}
$$

where $\overline{\mathrm{D}}$ is defined in terms of the lamina material properties (see Table 1) and plate thickness $h$ by

$$
\bar{D}=\frac{\sqrt{E_{L} E_{T}} h^{3}}{12\left(1-v_{L T} v_{T L}\right)}
$$

This bending stiffness is used herein to permit the buckling performance of laminates that are made of the same material, but with different ply orientations, to be compared directly. Two groups of curves are shown in the figure; the dashed and solid curves correspond to results for clamped and simply supported plates, respectively. Four curves appear within each group that correspond to values of the compliance ratio given by $R_{2}=0$ (rigidly restrained), $0.1,0.5$, and $\infty$. The results for $R_{2}=\infty$ correspond to an unrestrained plate for which $\mathrm{N}_{\mathrm{y} 1}=\mathrm{N}_{\mathrm{xy} 1}=$ 0 .

The results in Fig. 3 show a monotonic reduction in the nondimensional buckling load as the number of plies $(8 \mathrm{~m})$ increases. However, because $\bar{D}$ increases with the cube of the plate thickness h, the actual buckling load increases by the factor $\mathrm{m}^{3}$ as the number of plies increases. The results in Fig. 3 also show a reduction in the buckling load as the compliance ratio decreases, for both simply supported and clamped plates, as expected. Specifically, the unrestrained plates, that experience uniaxial compression, exhibit the highest buckling loads and the corresponding rigidly restrained plates, that experience biaxial compression, exhibit the lowest buckling loads.

The corresponding buckling interaction curves for 
the axially stiff $\left[\left( \pm 45 / 0_{2}\right)_{\mathrm{m}}\right]_{\mathrm{s}}$ laminates of Fig. 3 with $\mathrm{m}$ $=1$ ( 8 plies $)$ and $m=6$ (48 plies) are shown in Fig. 4 . The dashed and solid curves correspond to results for clamped and simply supported plates, respectively. In addition, the horizontal, flat portions of the curves correspond to wide-column buckling modes for infinitely long plates. Points on the curves that correspond to values of $\mathrm{R}_{2}=0$ (rigidly restrained), $0.1,0.5,1,10$, and $\infty$ (unrestrained) are indicated by six different symbols. These points are located by noting that the slope of a line that emanates from the origin in the figure, is given by

$$
\frac{\mathrm{N}_{\mathrm{y} 1}}{\mathrm{~N}_{\mathrm{x} 1}^{\mathrm{c}}}=\frac{\mathrm{v}_{\mathrm{yx}}}{1+\mathrm{R}_{2}}
$$

For these axially stiff laminates, $v_{y x}=0.193$. Additionally, the 8-ply plates have a much higher degree of flexural anisotropy $(\gamma=0.18, \delta=0.21)$ than the 48-ply plates $(\gamma=0.01, \delta=0.02)$. These results indicate that the rigidly restrained plates $\left(R_{2}=0\right)$ experience the largest amount of transverse compression, as expected.

Buckling interaction curves for $\left[\left( \pm 45 / 0_{2}\right)_{2}\right]_{s},[( \pm 45 /$ $\left.\left.90_{2}\right)_{2}\right]_{s}$, and $\left[( \pm 45 / 0 / 90)_{2}\right]_{s}$ 16-ply laminates made of the IM7/5260 material are presented in Fig. 5 for simply supported (solid lines) and clamped (dashed lines) boundary conditions. Points on the curves for values of $R_{2}=0$ (rigidly restrained), 0.1, 0.5, 1, 10, and $\infty$ (unrestrained) are also indicated by six different symbols. These points are also located by noting that the slope of a line that emanates from the origin in the figure, is given by Eq. (51). Values of $v_{\mathrm{yx}}$ for these laminates, to be used with Eq. (51), are given in Table 2. Like for Fig. 4, the horizontal, flat portions of the curves correspond to wide-column buckling modes.

The results in Fig. 5 show the basic effects of ply orientation on the buckling resistance of the 16-ply laminates, as the laminate configuration changes from axially stiff to quasi-isotropic to transversely stiff. In general, the transversely stiff $\left[\left( \pm 45 / 90_{2}\right)_{2}\right]_{s}$, plate exhibits the greatest buckling resistance for states of biaxial compression. In contrast, the axially stiff $\left[\left( \pm 45 / 0_{2}\right)_{2}\right]_{\mathrm{s}}$ plate exhibits the lowest buckling resistance for states of biaxial compression. For a state of uniaxial compression, the quasi-isotropic laminate exhibits the greatest buckling resistance. For all cases, the clamped plates are more buckling resistant than the simply supported plates, as expected. Moreover, the simply supported plates exhibit wide-column modes for the smaller values of the compliance ratio $R_{2}$ designated by the symbols, whereas none of the clamped plates exhibit wide-column modes.

A comparison of the structural efficiency of 16-ply $\left[( \pm 45 / 0 / 90)_{2}\right]_{\mathrm{s}}$ quasi-isotropic plates with simply sup- ported edges is presented in Fig. 6. Each curve in this figure corresponds to one of the nine material systems defined in Table 1. A thick, solid gray curve is also shown for plates made of aluminum with an elastic modulus $\mathrm{E}=10^{6} \mathrm{psi}$, a Poisson's ratio $v=0.33$, and a density $\rho_{\mathrm{Al}}=0.1 \mathrm{lb} / \mathrm{in}^{3}$. In this figure, the buckling loads are normalized by the bending stiffness $\mathrm{D}_{\mathrm{Al}}$, which is obtained by substituting the properties for the aluminum material into Eq. (50). Moreover, the nondimensional buckling loads are weighted by the density ratio $\rho_{\mathrm{Al}} / \rho$, where $\rho$ is the density of the material (see Table 1) that corresponds to a given curve in the figure. Thus, plates with higher buckling resistance per unit mass are represented by curves that are farther from the origin of the graph. Also, points on the curves that correspond to values of $R_{2}=0$ (rigidly restrained), 0.1, 0.5, 1, 10, and $\infty$ (unrestrained) are indicated by symbols.

The results in Fig. 6 show that all the materials out perform the aluminum material except the Kevlar 49-epoxy and S-glass-epoxy materials. The best performance is exhibited by the $\mathrm{P}-100 / 3502$ pitch-epoxy material, followed by the boron-aluminum material. The worst performance is exhibited by the S-glass-epoxy material, followed by the Kevlar 49-epoxy material. The symbols shown in the figure indicate a very pronounced effect of lateral edge restraint, which varies somewhat with material system.

Results are presented in Fig. 7 for $\left[( \pm \theta)_{\mathrm{m}}\right]_{\mathrm{s}}$ balanced, angle-ply laminates that dramatize the effects of fiber orientation and material system on the induced lateral load $\mathrm{N}_{\mathrm{y} 1}$. Each curve in this figure also corresponds to one of the nine material systems defined in Table 1 , and is independent of the number of laminate plies. Moreover, the results are applicable to an infinite range of compliance ratios given by $0 \leq R_{2}<\infty$. The results in Fig. 7 show the largest variation in the load ratio $\mathrm{N}_{\mathrm{y} 1} / \mathrm{N}_{\mathrm{x} 1}^{\mathrm{c}}$ in the approximate range of $50 \mathrm{deg}<\theta<80$ deg. The greatest variations in the load ratio are exhibited by the laminates made of the $\mathrm{P}-100 / 3502$ pitch-epoxy material, followed by those made of the IM7/PETI-5 material. In contrast, the smallest variations are exhibited by the laminates made of the boron-aluminum material, followed by those made of the S-glass-epoxy material. It is important to note that the induced lateral load for many of the laminate configurations exceeds the magnitude of the applied load; the largest being about 3.3 times the applied load.

The effects of fiber orientation and lateral edge restraint on the buckling resistance of 4-ply, highly anisotropic $[ \pm \theta]_{\mathrm{S}}$ laminates made of the IM7/5260 material given in Table 1 are shown in Fig. 8. In particular, the nondimensional buckling load defined by Eq. (49) is giv- 
en as a function of the fiber angle $\theta$. Two groups of curves are shown in the figure. The dashed and solid curves correspond to results for clamped and simply supported plates, respectively. In addition, four curves appear within each group that correspond to values of the compliance ratio given by $R_{2}=0$ (rigidly restrained), $0.1,0.5$, and $\infty$ (unrestrained, $\mathrm{N}_{\mathrm{y} 1}=\mathrm{N}_{\mathrm{xy} 1}=0$ ).

The results in Fig. 8 show a big effect of fiber orientation and lateral edge restraint. Unlike the corresponding results for the load ratio shown in Fig. 7, the largest variation in buckling load is in the approximate range of $25 \mathrm{deg}<\theta<70 \mathrm{deg}$. Generally, the results indicate that the clamped plates are more buckling resistant than the simply supported plates, as expected, but for several values of $\theta$, the unrestrained, simply supported plates are more buckling resistant than the corresponding clamped plates with $\mathrm{R}_{2}=0$ (rigidly restrained) and $\mathrm{R}_{2}=$ 0.1 . This somewhat surprising result illustrate a detrimental effect of the biaxial compression state that is induced by severely restraining the lateral movement of the clamped-plate edges. Thus, neglecting the effects of inplane restraint in a preliminary-design buckling analysis could lead to an erroneous representation of the true response and negative margins of safety.

The results in Fig. 8 also show significant differences in the shapes of the curves for the corresponding clamped and simply supported plates with $R_{2}=0,0.1$, and 0.5 and in the range of approximately $25 \mathrm{deg}<\theta<$ $65 \mathrm{deg}$. Insight into these differences is obtained by examining the correponding buckling interaction curves that are presented in Figs. 9 and 10 for the simply supported and clamped $[ \pm \theta]_{\mathrm{s}}$ laminates, respectively, made of the IM7/5260 material. Five curves are shown in Fig. 9, and in Fig. 10, that correspond to values of $\theta=15,30$, 45,60 , and $75 \mathrm{deg}$. Also, points on the curves that correspond to values of $R_{2}=0$ (rigidly restrained), $0.1,0.5$, 1,10 , and $\infty$ (unrestrained) are indicated by six different symbols. Comparison of the symbols shown in Fig. 9 for the simply supported plates indicate wide-column buckling modes for values of $\theta=30,45$, and $60 \mathrm{deg}$. For the remaining values of $\theta$, the buckling modes are not widecolumn modes. In addition, none of the modes for the clamped plates with the values of $R_{2}$ indicated by the symbols are wide-column modes. Thus, the difference in the shape of the curves in Fig. 8, for the clamped and simply supported plates with lateral edge restraint, appear to be associated with the difference in mode shapes.

\section{$\underline{\text { Results for Unbalanced Laminates }}$}

For unbalanced laminates, inplane shearing deformations will develop under uniaxial compression loading, unless rigidly restrained, because the shear- extensional coupling coefficients are nonzero; that is, $\mathrm{a}_{16}$ $\neq 0$ and $\mathrm{a}_{26} \neq 0$ (see Eq. (25a). The induced loads associated with restraining lateral movement and inplane shearing deformations are given by Eqs. (34) and (35), or by Eqs. (36) and (37). Two special cases that will be addressed subsequently are the cases in which only the lateral inplane movement of a plate is restrained $\left(\mathrm{R}_{3} \rightarrow \infty\right)$ and the case in which only the shear deformation of a plate is restrained $\left(R_{2} \rightarrow \infty\right)$. The induced loads for the first case are defined by Eqs. (40) and (41), and those for the second case are defined by Eqs. (42) and (43).

Results are presented in Table 3 that show the load ratio $\left(1+R_{2}\right) \frac{N_{y 1}}{N_{x 1}^{c}}$, or equivalently, $v_{y x}$ for $\left[\left(+45_{2} / 0_{2}\right)_{m}\right]_{s}$, $\left[\left(+45_{2} / 90_{2}\right)_{\mathrm{m}}\right]_{\mathrm{s}}$, and $\left[\left(+45_{2} / 0 / 90\right)_{\mathrm{m}}\right]_{\mathrm{s}}$ unbalanced laminates made from one of the nine different material systems given in Table 1 and for the case in which only the lateral inplane movement of a plate is restrained $\left(\mathrm{R}_{3} \rightarrow \infty\right)$; that is, inplane shear deformations are unrestrained and $\mathrm{N}_{\mathrm{xy} 1}$ $=0$. The results are independent of the number of laminate plies and indicate that the $\left[\left(+45_{2} / 90_{2}\right)_{\mathrm{m}}\right]_{\mathrm{s}}$ laminates exhibit the largest values of $v_{\mathrm{yx}}$ for a given material system; the smallest values are exhibited by the $\left[\left(+45_{2} / 0_{2}\right)_{\mathrm{m}}\right]_{\mathrm{s}}$ laminates. Moreover, for the $\left[\left(+45_{2} / 90_{2}\right)_{\mathrm{m}}\right]_{\mathrm{s}}$ laminates, the largest value of $v_{\mathrm{yx}}$ is obtained for the Kevlar 49-epoxy material. For the $\left[\left(+45_{2} / 0 / 90\right)_{\mathrm{m}}\right]_{\mathrm{s}}$ and $\left[\left(+45_{2} / 0_{2}\right)_{\mathrm{m}}\right]_{\mathrm{s}}$ laminates, the largest value of $v_{\mathrm{yx}}$ is obtained for the boron-aluminum material. For all the laminates and material systems, $0<\mathrm{N}_{\mathrm{y} 1}<\mathrm{N}_{\mathrm{x} 1}^{\mathrm{c}}$ for all allowable values of the compliance ratio $\mathrm{R}_{2}$ and the laminates experience a state of uniform biaxial compression.

Results are presented in Table 4 that show the load ratio $\left(1+\mathrm{R}_{3}\right) \frac{\mathrm{N}_{\mathrm{xy} 1}}{\mathrm{~N}_{\mathrm{x} 1}^{\mathrm{c}}}$, or equivalently, $\eta_{\mathrm{x}, \mathrm{xy}}$ for $\left[\left(+45_{2} /\right.\right.$ $\left.\left.0_{2}\right)_{\mathrm{m}}\right]_{\mathrm{s}},\left[\left(+45_{2} / 90_{2}\right)_{\mathrm{m}}\right]_{\mathrm{s}}$, and $\left[\left(+45_{2} / 0 / 90\right)_{\mathrm{m}}\right]_{\mathrm{s}}$ laminates made from one of the nine different material systems and for the case in which only the inplane shear deformation of a plate is restrained $\left(\mathrm{R}_{2} \rightarrow \infty\right)$; that is, inplane lateral movements are unrestrained and $\mathrm{N}_{\mathrm{y} 1}=0$. These results, all negative, are also independent of the number of laminate plies and indicate that the $\left[\left(+45_{2} / 90_{2}\right)_{\mathrm{m}}\right]_{\mathrm{s}}$ laminates exhibit the largest magnitudes of $\eta_{x, x y}$ for a given material system. The smallest magnitudes of $\eta_{x, x y}$ are exhibited by the $\left[\left(+45_{2} / 0_{2}\right)_{\mathrm{m}}\right]_{\mathrm{s}}$ laminates. Moreover, for the $\left[\left(+45_{2} / 90_{2}\right)_{\mathrm{m}}\right]_{\mathrm{s}}$ and $\left[\left(+45_{2} / 0 / 90\right)_{\mathrm{m}}\right]_{\mathrm{s}}$ laminates, the largest magnitude of $\eta_{x, x y}$ is obtained for the $\mathrm{P}-100 / 3502$ pitchepoxy material. For the $\left[\left(+45_{2} / 0_{2}\right)_{\mathrm{m}}\right]_{\mathrm{s}}$ laminate, the largest magnitude of $\eta_{x, x y}$ is obtained for the S-glass-epoxy material. For all the laminates and material systems, and for all allowable values of the compliance ratio $R_{3}$, the 
laminates experience a state of uniform axial compression and negative shear (see Fig. $2 b$ for the positiveshear direction). The presence of negative shear is rationalized by the fact that the 45-deg plies tend to rotate counterclockwise in the $x-y$ plane when the plates are subjected to the uniaxial compression load and shear deformations are not restrained. Thus, the restoring force of the restraining medium must act to rotate these plies in the opposite direction, which implies the presence of negative shear stresses. Although not addressed specifically in the present study, the results in Table 4 indicate the possibility of buckling under uniaxial tension loads when only the inplane shear deformation of an unbalanced laminate are restrained.

Graphs of the load ratios $\mathrm{N}_{\mathrm{y} 1} / \mathrm{N}_{\mathrm{x} 1}^{\mathrm{c}}$ and $-\mathrm{N}_{\mathrm{xy} 1} / \mathrm{N}_{\mathrm{x} 1}^{\mathrm{c}}$ are presented in Figs. 11 and 12, respectively, for $\left[\left(+45_{2} /\right.\right.$ $\left.\mathrm{O}_{2}\right)_{\mathrm{m}} \mathrm{J}_{\mathrm{s}}$ laminates made of the IM7/5260 material defined in Table 1. These results are independent of the number of laminate plies and are given as a function of the compliance ratio $R_{2}$ by the black curves, for values of $R_{3}=0$ (rigidly restrained against inplane shear deformation), $0.1,0.5,1,10$, and $\infty$ (unrestrained against inplane shear deformation). The dashed gray curves in the figures correspond to similar results in which the compliance ratios are equal; that is, $\mathrm{R}_{2}=\mathrm{R}_{3}$. The circular symbol on the ordinate of the graphs correspond to results in which the plates are rigidly restrained against all inplane movement.

The results in Figs. 11 and 12 define a very broad spectrum of combined biaxial compression and negative shear loads that can result from restraining the inplane expansion, contraction, and shear deformation of the plates, for a very general variety of restraint combinations. For all restraint scenaros represented in the figures, the induced loads diminish as either of the restraint conditions is relaxed. Moreover, the magnitudes of the induced loads never exceed $20 \%$ of the magnitude of the applied axial compression for this family of laminates.

The effects of changing material system on the load ratios $\mathrm{N}_{\mathrm{y} 1} / \mathrm{N}_{\mathrm{x} 1}^{\mathrm{c}}$ and $-\mathrm{N}_{\mathrm{xy} 1} / \mathrm{N}_{\mathrm{x} 1}^{\mathrm{c}}$ are presented in Figs. 13 and 14 , respectively, for the $\left[\left(+45_{2} / 0_{2}\right)_{\mathrm{m}}\right]_{\mathrm{s}}$ laminates $(\mathrm{m}=$ $1,2, \ldots)$. In particular, curves that show the load ratios as a function of the compliance ratio $\mathrm{R}$ are presented, where $R_{2}=R_{3}=R$. Values of $R=0$ and $R=\infty$ correspond to plates that are rigidly restrained and unrestained against inplane deformation, respectively. Each curve shown in these two figures corresponds to one of the nine material systems defined by Table 1. Suprizingly, the results in Fig. 13 show that the boron-aluminum and the P-100/ 3502 pitch-epoxy materials exhibit the largest and smallest values of $\mathrm{N}_{\mathrm{y} 1} / \mathrm{N}_{\mathrm{x} 1}^{\mathrm{c}}$ for the range of $\mathrm{R}$ shown. Also, the magnitudes of $\mathrm{N}_{\mathrm{y} 1} / \mathrm{N}_{\mathrm{x} 1}^{\mathrm{c}}$ never exceed $25 \%$ of the magnitude of the applied axial compression for these laminates and tend to diminish rapidly with increasing values of the compliance ratio $\mathrm{R}$. In contrast, the results in Fig. 14 show that both the boron-aluminum and P-100/ 3502 pitch-epoxy materials exhibit the smallest magnitudes of $\mathrm{N}_{\mathrm{xy} 1} / \mathrm{N}_{\mathrm{x} 1}^{\mathrm{c}}$ for the range of $\mathrm{R}$ shown. The other materials exhibit, for the most part, the larger, nearly equal magnitudes of the induced negative-shear load. The magnitudes of $\mathrm{N}_{\mathrm{xy} 1} / \mathrm{N}_{\mathrm{x} 1}^{\mathrm{c}}$ never exceed $20 \%$ of the magnitude of the applied axial compression for these laminates and also tend to diminish rapidly with increasing values of the compliance ratio $\mathrm{R}$.

The effects of laminate stacking sequence and compliance ratio $\mathrm{R}\left(\mathrm{R}_{2}=\mathrm{R}_{3}=\mathrm{R}\right)$ on the load ratios $\mathrm{N}_{\mathrm{y} 1} / \mathrm{N}_{\mathrm{x} 1}^{\mathrm{c}}$ and $-\mathrm{N}_{\mathrm{xy} 1} / \mathrm{N}_{\mathrm{x} 1}^{\mathrm{c}}$ are illustrated in Fig. 15 for the $\left[\left(+45_{2} /\right.\right.$ $\left.\left.0_{2}\right)_{\mathrm{m}}\right]_{\mathrm{s}},\left[\left(+45_{2} / 90_{2}\right)_{\mathrm{m}}\right]_{\mathrm{s}}$, and $\left[\left(+45_{2} / 0 / 90\right)_{\mathrm{m}}\right]_{\mathrm{s}}$ laminates made of the IM7/5260 material. The solid and dashed curves correspond to results for $\mathrm{N}_{\mathrm{y} 1} / \mathrm{N}_{\mathrm{x} 1}^{\mathrm{c}}$ and $-\mathrm{N}_{\mathrm{xy} 1} / \mathrm{N}_{\mathrm{x} 1}^{\mathrm{c}}$, respectively. Moreover, the gray solid and dashed curves correpond to results for the $\left[\left(+45_{2} / 0 / 90\right)_{\mathrm{m}}\right]_{\mathrm{s}}$ laminates. Like the previous two figures, the results show a decline in the magnitudes of the load ratio with increasing values for the compliance ratio $\mathrm{R}$. The decline is the most pronounced for the $\left[\left(+45_{2} / 90_{2}\right)_{\mathrm{m}}\right]_{\mathrm{s}}$ laminates and the least pronounced for the $\left[\left(+45_{2} / 0_{2}\right)_{\mathrm{m}}\right]_{\mathrm{s}}$ laminates. These results also show that placing more laminate fibers perpendicular to the axis of the applied load dramatically amplifies the magnitudes of the induced compression and shear loads. The largest magnitudes of the load ratios are on the order of $60 \%$ to $70 \%$ of the applied load, for the rigidly restrained $\left[\left(+45_{2} / 90_{2}\right)_{\mathrm{m}}\right]_{\mathrm{s}}$ laminates.

Nondimensional buckling loads, defined by Eq. (49), are shown in Fig. 16 as a function of the number of laminate plies for the $\left[\left(+45_{2} / 90_{2}\right)_{\mathrm{m}}\right]_{\mathrm{s}}$ laminates made of the IM7/5260 material. Two groups of curves are shown in the figure; the dashed and solid curves correspond to results for clamped and simply supported plates, respectively. Four curves also appear within each group that correspond to different values of the compliance ratio $\mathrm{R}$, where $R_{2}=R_{3}=R$. Specifically, the values are $R=0$ (rigidly restrained), $0.1,0.5$, and $\infty$. The results for $\mathrm{R}=$ $\infty$ correspond to an unrestrained plate for which $\mathrm{N}_{\mathrm{y} 1}=$ $\mathrm{N}_{\mathrm{xy} 1}=0$.

Unlike the results shown in Fig. 3 for the $[( \pm 45 /$ $\left.\left.0_{2}\right)_{\mathrm{m}}\right]_{\mathrm{s}}$ balanced laminates (note that $\mathrm{R}_{3}=0$ and $\mathrm{R}_{2}=\mathrm{R}$ for the balanced laminates), the results in Fig. 16 for the $\left[\left(+45_{2} / 90_{2}\right)_{\mathrm{m}}\right]_{\mathrm{s}}$ unbalanced laminates show a monotonic increase in the nondimensional (and actual) buckling load as the number of plies increases, and show a reversal in the response trend for the clamped plates with $\mathrm{R}=$ $\infty$. Specifically, the unrestrained clamped plates exhibit 
lower buckling loads than the restrained clamped plates, which at first glance appears to be counterintuitive. The results for the clamped plates with the other values for $\mathrm{R}$, and all the results for the simply supported plates show a reduction in the buckling load as the compliance ratio decreases, which agrees with the trend exhibited by the $\left[\left( \pm 45 / 0_{2}\right)_{\mathrm{m}}\right]_{\mathrm{s}}$ balanced laminates (Fig. 3). However, it is important to note that the unbalanced laminates have a relatively high degree of flexural anisotropy which can greatly affect the manner in which combined loads interact (e.g., see Ref. 3).

Insight into the combined-load interactions experienced by the $\left[\left(+45_{2} / 90_{2}\right)_{\mathrm{m}}\right]_{\mathrm{s}}$ unbalanced laminates (Fig. 16) can be obtained by examining the buckling interaction curves and surfaces for a general state of biaxial compression and shear. Toward this goal, buckling interaction curves for 16-ply $\left[\left(+45_{2} / 90_{2}\right)_{2}\right]_{\mathrm{s}}$ laminates made of the IM7/5260 material are shown in Figs. 17 and 18.

In Fig. 17, $\mathrm{N}_{\mathrm{y} 1}$ versus $\mathrm{N}_{\mathrm{x} 1}^{\mathrm{c}}$ buckling interaction curves are presented, where the gray and black curves correspond to results for clamped and simply supported plates, respectively. Moreover, the dashed and solid curves correspond to results for negative and nonnegative shear loads, respectively. Furthermore, the horizontal, flat portions of the curves correspond to widecolumn buckling modes for infinitely long plates. In Fig. 18 , the companion $\mathrm{N}_{\mathrm{xy} 1}$ versus $\mathrm{N}_{\mathrm{x} 1}^{\mathrm{c}}$ buckling interaction curves are presented, but only for the simply supported plates. Points on the curves of both figures that correspond to several selected values of the compliance ratios $R_{2}$ and $R_{3}$ are also shown by ten different symbols, and include the cases shown in Fig. 16. The values for both $\mathrm{R}_{2}$ and $\mathrm{R}_{3}$ shown in Fig. 17 and 18 include 0 (rigidly restrained), 0.1, 0.5, 1, 2, 5, 10, and $\infty$ (unrestrained). The points in Figs. 17 and 18 are located by noting that the slope of a line that emanates from the origin in the figures, is given by $\mathrm{N}_{\mathrm{y} 1} / \mathrm{N}_{\mathrm{x} 1}^{\mathrm{c}}$ and $\mathrm{N}_{\mathrm{xy} 1} / \mathrm{N}_{\mathrm{x} 1}^{\mathrm{c}}$, respectively.

It is important to point out that the buckling interaction curves in Figs. 17 and 18 are not level curves of the corresponding buckling interaction surfaces; that is, they are not the curves formed by the intersection of a plane that is parallel to one of the coordinate planes with the surface. To illustrate this point, consider the buckling interaction surfaces depicted in Figs. 19 and 20. The level curves that correspond to constant values of $\mathrm{N}_{\mathrm{y} 1}$ are parabola-like curves that relate $\mathrm{N}_{\mathrm{x} 1}^{\mathrm{c}}$ and $\mathrm{N}_{\mathrm{xy} 1}$. These parabola-like curves are not symmetric about the plane $\mathrm{N}_{\mathrm{xy} 1}=0$ because the relatively high degree of flexural anisotropy $(\gamma=0.56, \delta=0.37)$ exhibited by the 16-ply $\left[\left(+45_{2} / 90_{2}\right)_{2}\right]_{\mathrm{s}}$ laminates. The top-most level curve encloses the region of the buckling interaction surface that corresponds to wide-column modes. The level curves that correspond to constant values of $\mathrm{N}_{\mathrm{xy} 1}$, are similar in shape to the curves shown in Fig. 17, but have different proportions. The curves shown in Fig. 17 are obtained by first defining the plane given by a specific value for $\mathrm{N}_{\mathrm{xy} 1} / \mathrm{N}_{\mathrm{x} 1}^{\mathrm{c}}$ and then determining the curve $e$ in Fig. 19 that is the intersection of that given plane with the buckling interaction surface. Then, the curve $e$ is projected onto the $\mathrm{N}_{\mathrm{y} 1}-\mathrm{N}_{\mathrm{x} 1}^{\mathrm{c}}$ plane (dashed curve) to obtain a buckling interaction curve like those presented in Fig. 17.

The buckling interaction curves in Fig. 18 are obtained by first defining the plane given by a specific value for $\mathrm{N}_{\mathrm{y} 1} / \mathrm{N}_{\mathrm{x} 1}^{\mathrm{c}}$ and then determining the curve $\mathcal{C}$ in Fig. 20 , that is the intersection of that given plane with the buckling interaction surface. Then, the curve $e$ is projected onto the $\mathrm{N}_{\mathrm{xy} 1}-\mathrm{N}_{\mathrm{x} 1}^{\mathrm{c}}$ plane (dashed curve) to obtain a buckling interaction curve like those presented in Fig. 18. Note, that as the value for $\mathrm{N}_{\mathrm{y} 1} / \mathrm{N}_{\mathrm{x} 1}^{\mathrm{c}}$ increases, eventually the plane will intersect the plane of wide-column buckling modes and yield a buckling interaction curve with a flat section like those shown in Fig. 18 for $\mathrm{N}_{\mathrm{y} 1} / \mathrm{N}_{\mathrm{x} 1}^{\mathrm{c}}=0.6$ and 0.8 .

The results in Fig. 17 indicate that curves for the simply supported plates have much smaller slopes than the corresponding curves for the clamped plates, that is caused to a large extent by the higher wide-column buckling resistance of the clamped plates. Moreover, there is more distance separating the curves for the clamped plates than for the simply supported plates. Thus, for a given value of $\mathrm{N}_{\mathrm{y} 1} / \mathrm{N}_{\mathrm{x} 1}^{\mathrm{c}}$, the corresponding line emanating from the origin intersects the buckling interaction curves for the simply supported plate in a region with a much different geometrical character than the corresponding region for the clamped plates. This difference in geometrical character accounts for the fact that $\left(\mathrm{N}_{\mathrm{x} 1}^{\mathrm{c}}\right)_{\text {cr }}$ for the rigidly restrained plate $\left(\mathrm{R}_{2}=\mathrm{R}_{3}=\mathrm{R}=0\right)$ is less than $\left(\mathrm{N}_{\mathrm{x} 1}^{\mathrm{c}}\right)_{\mathrm{cr}}$ for the corresponding unrestrained plate $\left(R_{2}=R_{3}=R=\infty\right)$ when the edges are simply supported, and that this relationship reverses when the edges are clamped.

Figure 18 gives a clear indication of how the flexural anisotropy interacts with the combined loads to change the geometrical character of the buckling interaction surface. In particular, a basic effect of the flexural anisotropy, as exhibited by the curve for $\mathrm{N}_{\mathrm{y} 1} / \mathrm{N}_{\mathrm{x} 1}^{\mathrm{c}}=0$, is the lack of symmetry about the plane $\mathrm{N}_{\mathrm{xy} 1}=0$. In fact, the corresponding curve in which the flexural anisotropy is neglected is obtained by translating this curve vertically until it is symmetric about the plane $\mathrm{N}_{\mathrm{xy} 1}=0$. As the 
value for $\mathrm{N}_{\mathrm{y} 1} / \mathrm{N}_{\mathrm{x} 1}^{\mathrm{c}}$ increases, the curves in Fig. 18 skew vertically towards the plane $\mathrm{N}_{\mathrm{xy} 1}=0$, which suggests that the importance of the flexural anisotropy is being mitigated, at least somewhat, by the increase in the lateral compression load.

The effects of laminate stacking sequence and compliance ratio $R\left(R_{2}=R_{3}=R\right)$ on the nondimensional buckling load are illustrated in Fig. 21 by results for the $\left[\left(+45_{2} / 0_{2}\right)_{\mathrm{m}}\right]_{\mathrm{s}}, \quad\left[\left(+45_{2} / 90_{2}\right)_{\mathrm{m}}\right]_{\mathrm{s}}$, and $\left[\left(+45_{2} / 0 / 90\right)_{\mathrm{m}}\right]_{\mathrm{s}}$ laminates made of the IM7/5260 material. The solid black, solid gray, and dashed black curves correspond to results for the $\left[\left(+45_{2} / 90_{2}\right)_{\mathrm{m}}\right]_{\mathrm{s}},\left[\left(+45_{2} / 0_{2}\right)_{\mathrm{m}}\right]_{\mathrm{s}}$, and $\left[\left(+45_{2} / 0 / 90\right)_{\mathrm{m}}\right]_{\mathrm{s}}$ laminates, respectively. Two curves are given for each laminate that correspond to rigidly restrained $(R=0)$ and unrestrained $(R=\infty)$ edges. These results show a monotonic increase in the nondimensional buckling load with an increase in the number of plies, except for the rigidly restrained $\left[\left(+45_{2} / 0_{2}\right)_{\mathrm{m}}\right]_{\mathrm{s}}$ laminate. In addition, the greater buckling resistance is generally exhibited by the unrestrained $\left[\left(+45_{2} / 0 / 90\right)_{\mathrm{m}}\right]_{\mathrm{S}}$ laminates and the least by the rigidly restrained $\left[\left(+45_{2} / 90_{2}\right)_{\mathrm{m}}\right]_{\mathrm{s}}$ laminates. Moreover, the greatest influence of the compliance ratio, as evidenced by the separation in the two curves for each laminate, is exhibited by the $\left[\left(+45_{2} / 90_{2}\right)_{\mathrm{m}}\right]_{\mathrm{s}}$ laminates.

As mentioned previously, $\left[+\theta_{3} / 15\right]_{\mathrm{S}}$ unbalanced laminates exhibit a wide range of values for the overall laminate Poisson's ratio $v_{\mathrm{yx}}$ that includes negative values. This characteristic is illustrated in Fig. 22 for the nine material systems given in Table 1 . These results indicate positive values of $v_{\mathrm{yx}}$ for all the laminate configurations made of the boron-aluminum and S-glass-epoxy materials. For the laminate configurations with negative values of $v_{\mathrm{yx}}$, the magnitudes appear to be the largest in the region defined approximately by $60 \mathrm{deg}<\theta<90$ deg. The negative values with the largest magnitudes are generally exhibited by the laminates made of the P-100/ 3502 pitch-epoxy material, followed by those made of the IM7/PETI-5 material. When applying these results to the case in which only the lateral inplane movement of a plate is restrained $\left(\mathrm{R}_{3} \rightarrow \infty, \mathrm{N}_{\mathrm{xy} 1}=0\right)$, defined by Eqs. (40) and (41), it is seen that a lateral tension load $\left(\mathrm{N}_{\mathrm{y} 1}<\right.$ 0 ) exists when $v_{y x}<0$, and that values of $\theta$ exist for several of the material systems where $v_{y x}$ is near zero and $\mathrm{N}_{\mathrm{y} 1}$ is negligible.

The load ratios $\mathrm{N}_{\mathrm{y} 1} / \mathrm{N}_{\mathrm{x} 1}^{\mathrm{c}}$ and $-\mathrm{N}_{\mathrm{xy} 1} / \mathrm{N}_{\mathrm{x} 1}^{\mathrm{c}}$ are presented in Figs. 23 and 24 for rigidly restrained $\left[+\theta_{3} / 15\right]_{\mathrm{S}}$ plates $\left(R_{2}=R_{3}=0\right)$ made from the nine material systems considered herein. For this case, the induced loads are given by Eqs. (38) and (39). These results indicate positive values for $\mathrm{N}_{\mathrm{y} 1} / \mathrm{N}_{\mathrm{x} 1}^{\mathrm{c}}$ over the range of fiber orientations considered, and indicate negative values for
$\mathrm{N}_{\mathrm{xy} 1} / \mathrm{N}_{\mathrm{x} 1}^{\mathrm{c}}$. The most and least pronounced variations in the load factors are exhibited by the laminates made of the P-100/3502 pitch-epoxy and the boron-aluminum materials, respectively. The largest magnitudes of $\mathrm{N}_{\mathrm{y} 1} / \mathrm{N}_{\mathrm{x} 1}^{\mathrm{c}}$ appear to be in the region defined approximately by $45 \mathrm{deg}<\theta<60 \mathrm{deg}$. Similarly, the largest magnitudes of $\mathrm{N}_{\mathrm{xy} 1} / \mathrm{N}_{\mathrm{x} 1}^{\mathrm{c}}$ appear to be in the region defined approximately by $40 \mathrm{deg}<\theta<60 \mathrm{deg}$. Altogether, the magnitudes of the load factors never exceed $60 \%$ of the applied axial load, for this family of laminates. When compared to the results in Fig. 22, that define $\mathrm{N}_{\mathrm{y1}} / \mathrm{N}_{\mathrm{x} 1}^{\mathrm{c}}$ for the case in which inplane shear deformation is unrestrained, the results in Figs. 23 and 24 illustrate very pronounced effects caused by restraining the inplane shearing deformations.

Two groups of curves are presented in Fig. 25 that show the effects of the compliance ratio $R$, where $R_{2}=R_{3}$ $=\mathrm{R}$, on the load ratios $\mathrm{N}_{\mathrm{y} 1} / \mathrm{N}_{\mathrm{x} 1}^{\mathrm{c}}$ and $-\mathrm{N}_{\mathrm{xy} 1} / \mathrm{N}_{\mathrm{x} 1}^{\mathrm{c}}$ for $\left[+\theta_{3} /\right.$ $15]_{\mathrm{s}}$ plates made of the IM7/5260 material. The black and gray curves in the figure correspond to results for $\mathrm{N}_{\mathrm{y} 1} / \mathrm{N}_{\mathrm{x} 1}^{\mathrm{c}}$ and $-\mathrm{N}_{\mathrm{xy} 1} / \mathrm{N}_{\mathrm{x} 1}^{\mathrm{c}}$, respectively. Moreover, each group has three curves that correspond to values of $\mathrm{R}=$ 0 (rigidly restrained), 0.05 , and 0.1 . The results in this figure show sizeable reductions in the induced loads for relatively small increases in the compliance ratio, with the greater reductions exhibited for the lateral load $\mathrm{N}_{\mathrm{y} 1}$. The results also show a slightly greater sensitivity to an increase in $\mathrm{R}$ for the induced lateral load than for the induce shear load, as evidenced by the difference in separation of the curves within each group. Moreover, the values of the fiber angle $\theta$ that corresponds to the maximum values of $\mathrm{N}_{\mathrm{y} 1} / \mathrm{N}_{\mathrm{x} 1}^{\mathrm{c}}$ and $\mathrm{N}_{\mathrm{xy} 1} / \mathrm{N}_{\mathrm{x} 1}^{\mathrm{c}}$ decrease slightly with increase in the compliance ratios. Overall, the magnitudes of the load factors never exceed $51 \%$ of the applied axial load.

Nondimensional buckling loads, defined by Eq. (49), are shown in Fig. 26 as a function of the fiber angle $\theta$ for the $\left[+\theta_{3} / 15\right]_{\mathrm{s}}$ laminates made of the IM7/5260 material. Two groups of curves are shown in the figure; the dashed and solid curves correspond to results for clamped and simply supported plates, respectively. Four curves also appear within each group that correspond to different values of the compliance ratio $R$, where $R_{2}=R_{3}$ $=\mathrm{R}$. Specifically, the values are $\mathrm{R}=0$ (rigidly restrained), 0.1, 0.5, and $\infty$ (unrestrained, $\mathrm{N}_{\mathrm{y} 1}=\mathrm{N}_{\mathrm{xy} 1}=0$ ).

The results in Fig. 26 show that the influence of the compliance ratio $\mathrm{R}$ varies significantly with the fiber angle $\theta$, and that the simply supported plates generally show a greater sensitivity to variations in $\mathrm{R}$ than do the clamped plates. Similarly, the simply supported plates exhibit much larger variations in the shape of the re- 
sponse curves, with variations in $\theta$, than do the clamped plates. Altogether, the results in Fig. 26 illustrate how the combined biaxial compression and shear loads that are present in the unbalanced laminates, restrained against inplane deformations, can produce counterintuitive response trends. For example, the results show that the unrestrained plates, that experience only uniaxial load, are less buckling resistant than the restrained plates in many cases. In fact, the restrained clamped plates are more buckling resistant than the unrestrained clamped plate for most of the values of the fiber angle $\theta$. Based on results presented previously herein, this seemingly unusual behavior is likely an artifice of the geometrical character of the corresponding buckling interaction surfaces, which is influenced to a large extent by flexural anisotropy.

\section{Generic Results and Example}

In developing buckling-design technology for laminated composite structures, it desirable to produce data and charts that have a broad range of applicability. This goal was accomplished in the present study by developing data for the elastically restrained, compression-loaded plates considered herein that can be used with the generic buckling-design charts presented in Refs. 1-3 and 18. In particular, the nondimensional buckling analysis described herein is formulated such that the buckling coefficient $\mathrm{K}_{\mathrm{x}}$ (see Eq. (18)), associated with the uniaxial compression load, is given in terms of the nondimensional orthotropy parameter $\beta$, the anisotropy parameters $\gamma$ and $\delta$, and the loading parameters $\mathrm{L}_{2}$ and $\mathrm{L}_{3}$ by

$$
\mathrm{K}_{\mathrm{x}}=\mathrm{K}_{\mathrm{x}}\left(\beta, \gamma, \delta, \mathrm{L}_{2}, \mathrm{~L}_{3}\right)
$$

for a given set of bending boundary conditions (e.g., simply supported and clamped edges). Examples of generic buckling-design charts like those presented in Refs. 1-3 and 18, and based on Eq. (52), are shown in Figs. 27 and 28. These figures give the buckling coefficient $\mathrm{K}_{\mathrm{x}}$ as a function of the nondimensional loading parameter $L_{2}$, for selected values of the parameters $\beta$ and $\mathrm{L}_{3}$, and for $\gamma=\delta=0.4$. The curves given by $\mathrm{K}_{\mathrm{x}}=\frac{1}{\mathrm{~L}_{2}}$ represent the wide-column buckling modes. Several similar figures are presented in Ref. 18 that are applicable to a wide range of laminate constructions and combined loading conditions. In addition, a large number of results that show the orthotropy parameter $\beta$ and the anisotropy parameters $\gamma$ and $\delta$ as a function of laminate construction are also found in Refs. 1-3 and 18. A sample of these results in shown in Fig. 29, which gives the anisotropy parameter $\gamma$ as function of the fiber angle $\theta$, and the nine material systems given in Table 1 , for $[+\theta]_{\mathrm{m}}$ unidirectional off-axis laminates.

To make use of generic results like those shown in Figs. 27-29, and to demonstrate the development of design data for the problem considered in the present study, the nondimensional load factors $\mathrm{L}_{2}$ and $\mathrm{L}_{3}$ were determined for the $[+\theta]_{\mathrm{m}}$ unidirectional off-axis laminates of Fig. 29. These results are shown in Figs. 30-33 for the nine material systems considered herein and are based on Eqs. (44) and (45). Moreover, the results given are for the special case in which the compliance ratios $R_{2}=R_{3}=$ R. For this simplification, Eqs. (44) and (45) reduce to

$$
\begin{aligned}
& \mathrm{L}_{2}=\frac{\mathrm{N}_{\mathrm{y} 1}}{\mathrm{~N}_{\mathrm{x} 1}^{\mathrm{c}}}\left(\frac{\mathrm{D}_{11}}{\mathrm{D}_{22}}\right)^{\frac{1}{2}}=\left[\frac{\mathrm{a}_{16} \mathrm{a}_{26}-\mathrm{a}_{12} \mathrm{a}_{66}(1+\mathrm{R})}{\mathrm{a}_{22} \mathrm{a}_{66}(1+\mathrm{R})^{2}-\mathrm{a}_{26}^{2}}\right]\left(\frac{\mathrm{D}_{11}}{\mathrm{D}_{22}}\right)^{\frac{1}{2}} \\
& { }_{3}=\frac{\mathrm{N}_{\mathrm{xy}}}{\mathrm{N}_{\mathrm{x} 1}^{\mathrm{c}}}\left(\frac{\mathrm{D}_{11}}{\mathrm{D}_{22}}\right)^{\frac{1}{4}}=-\left[\frac{\mathrm{a}_{12} \mathrm{a}_{26}-\mathrm{a}_{16} \mathrm{a}_{22}(1+\mathrm{R})}{\mathrm{a}_{22} \mathrm{a}_{66}(1+\mathrm{R})^{2}-\mathrm{a}_{26}^{2}}\right]\left(\frac{\mathrm{D}_{11}}{\mathrm{D}_{22}}\right)^{\frac{1}{4}}
\end{aligned}
$$

The results in Figs. 30 and 31 show the load factors $\mathrm{L}_{2}$ and $-\mathrm{L}_{3}$ for plates that are rigidly restrained against all inplane movement $(R=0)$. The negative of $L_{3}$ is used for the ordinate in Fig. 31 because the induced shear loads are negative for these laminates. The results show large variations in the load factors with the fiber angle $\theta$ and with material system. The largest variations are exhibited by the P-100/3502 pitch-epoxy material and the smallest by the boron-aluminum material. In addition, the curves shown in Fig. 30 for $\mathrm{L}_{2}$ exhibit symmetry about the line $\theta=45 \mathrm{deg}$, whereas the curves shown in Fig. 31 for $\mathrm{L}_{3}$ are asymmetric and generally exhibit maximums at different values of $\theta$.

The load factors $L_{2}$ and $-L_{3}$ for the $[+\theta]_{m}$ unidirectional off-axis laminates are shown in Figs. 32 and 33 for values of the compliance ratios given by $R=0.1$. This value for $\mathrm{R}$ means that the restraining medium has lateral and shear stiffnesses that are ten times the corresponding values for the plate. Comparing Figs. 30 with 32 and Fig. 31 with 33 , indicates that slightly relaxing the restraint of inplane deformations has a pronounced effect on the load factors $L_{2}$ and $L_{3}$. Specifically, the amplitudes of the curves are reduced substantially, the symmetry in the $\mathrm{L}_{2}{ }^{-}$ curves for $\mathrm{R}=0$ disappears, and the asymmetry in $\mathrm{L}_{3}$ curves becomes more pronounced.

\section{Example}

To illustrate the use of the generic figures that have been presented herein and in Refs. 1-3 and 18, consider a fully restrained $(R=0)$, simply supported $[+45]_{10}$ unidirectional, off-axis laminate made of the S-glass-epoxy material given in Table 1. From Fig. 25 in Ref. 17, one 
gets $\beta=1.8$. Similarly, from Fig. 29 herein and from Fig. 20 in Ref. 18 , one gets $\gamma=\delta \approx 0.4$. From Figs. 30 and 31 , one gets $L_{2} \approx 0.5$ and $L_{3} \approx-0.4$, respectively. Thus, the destabilizing loads are biaxial compression and negative shear loads. The value of $\mathrm{K}_{\mathrm{x}}$ is obtained from Figs. 27 and 28 by interpolating the results in the figures for $\beta=1.5$ and $\beta=2$, and for $L_{3}=0$ and $L_{3}=-0.5$. For $\beta=1.5, L_{2}=0.5$, and $L_{3}=0$, Fig. 27 gives $K_{x}=2.0$, which corresponds to a wide-column buckling mode. Similarly, for $\beta=1.5, L_{2}=0.5$, and $L_{3}=-0.5$, Fig. 27 also gives $\mathrm{K}_{\mathrm{x}}=2.0$. Therefore, $\mathrm{K}_{\mathrm{x}}=2.0$ for $\beta=1.5$ and $\mathrm{L}_{3}=-0.4$. Next, for $\beta=2, L_{2}=0.5$, and $L_{3}=0$, Fig. 28 also gives $\mathrm{K}_{\mathrm{x}}=2.0$ and a wide-column buckling mode. For $\beta=2$, $\mathrm{L}_{2}=0.5$, and $\mathrm{L}_{3}=-0.5$, Fig. 28 once again gives $\mathrm{K}_{\mathrm{x}}=2.0$ and a wide-column buckling mode. From these last two results, it follows that $\mathrm{K}_{\mathrm{x}}=2.0$ for $\beta=2$ and $\mathrm{L}_{3}=-0.4$. Finally, because $\mathrm{K}_{\mathrm{x}}=2.0$ for $\beta=1.5$ and $\mathrm{L}_{3}=-0.4$, and for $\beta=2$ and $L_{3}=-0.4$, it follows that $K_{x}=2.0$ for $\beta=1.8$ and $\mathrm{L}_{3}=-0.4$. Figure 29 in Ref. 17 indicates that $\mathrm{K}_{\mathrm{x}} \approx$ 4.5 for the corresponding unrestrained plate.

\section{Concluding Remarks}

An analytical approach for synthesizing buckling results and behavior for long, balanced and unbalanced symmetric laminates that are subjected to uniform axial compression and elastically restrained against inplane expansion or contraction and inplane shearing deformations has been presented. A nondimensional buckling analysis for long flexurally anisotropic plates that are subjected to combined loads has been described and useful nondimensional parameters have been presented. In particular, nondimensional parameters have been presented that represent the kinematically induced lateral and shear loads prior to buckling and that can be used to determine critical buckling loads for a wide range of laminate constructions. Moreover, the effects of membrane orthotropy and membrane anisotropy on the prebuckling stress state that is induced by restraining the inplane movement of a plate have been presented.

A large number of results have been presented herein for several selected laminates that are intended to highlight certain aspects of the behavioral trends and, to some extent, to facilitate a structural designer's transition to the use of the generic buckling design curves that are included in the paper. Additionally, several results have been presented that show the effects of laminate construction on the nature of the induced loads and the resulting buckling behavior. Results of this type could be important in the design of vehicles that must exploit plate anisotropy to achieve certain high-performance goals. Examples of generic buckling design curves have also been presented that provide physical insight into the buckling problem of the present paper in addition to providing useful design data. In addition, an example has been presented that demonstrates the use of the generic design curves. Overall, the analysis approach and results that have been presented identify the effects or characteristics of laminate membrane orthotropy and anisotropy, elastic inplane edge-movement restraint, and flexural orthotropy and anisotropy on laminated-plate buckling in a very general manner. Some seemingly counterintuitive response trends have been identified and explained by examining the geometrical character of buckling interaction surfaces for combined biaxial compression and shear loads. Moreover, the results have shown clearly that neglecting loads that are induced in compressionloaded plates by restraining the inplane movement of the unloaded edges may lead to very nonconservative representations of the true buckling resistance, particularly for laminates that exhibit shear-extensional coupling. Although the results are based on classical laminated-plate theory and have been demonstrated for infinitely long plates, the approach is applicable to more sophisticated plate theories that incorporate effects such as transverseshear flexibility. Many of the results can also be used in the analysis of finite-length plates.

\section{$\underline{\text { References }}$}

${ }^{1}$ Nemeth, M. P., "Buckling Behavior of Long Symmetrically Laminated Plates Subjected to Combined Loadings," NASA TP 3195, May 1992.

${ }^{2}$ Nemeth, M. P., "Buckling Behavior of Long Symmetrically Laminated Plates Subjected to Compression, Shear, and Inplane Bending Loads," AIAA Journal,Vol. 30, No. 12, December 1992, pp. 2959-2965.

${ }^{3}$ Nemeth, M. P., "Buckling Behavior of Long Anisotropic Plates Subjected to Combined Loads," NASA TP 3568, November 1995.

${ }^{4}$ Nemeth, M. P., "Buckling Behavior of Long Symmetrically Laminated Plates Subjected to Shear and Linearly Varying Axial Edge Loads," NASA TP 3659, July 1997.

${ }^{5}$ Harris, G. Z., "The Buckling of Orthotropic Rectangular Plates, Including the Effect of Lateral Edge Restraint," International Journal of Solids and Structures, Vol. 11, 1975, pp. 877-885.

${ }^{6}$ Obraztsov. I. F. and Vasil'ev, V.V., "Optimal Design of Composite Structures," Handbook of Composites, Vol. 2-Structure and Design, C.T. Herakovich and Y. M. Tarnopol'skii, eds., Elsevier Publishing Co., Amsterdam, 1989, pp. 3-84.

${ }^{7}$ Sherbourne, A. N. and Pandey, M. D., "Effects of In-Plane Restraint on the Stability of Laminated Composite Plates," Composite Structures, Vol. 20, 1992, pp. 73-81. 
${ }^{8}$ Miki, M. and Murotsu, Y., "Zero and Negative Poisson's Ratio of Laminated Fibrous Composites," Proc. 4th Japan-U.S. Conference on Composite Materials," Washington, D. C., Technomic Publishing Co., Lancaster, PA, 1988, pp. 538-547.

${ }^{9}$ Hine, P. J., Duckett, R. A., and Ward, I. M., "Negative Poisson's Ratios in Angle-Ply Laminates," Journal of Material Science Letters, Vol. 16, No. 7, 1997, pp. 541-544.

${ }^{10}$ Yeh, H.-L., Yeh, H.-Y., and Zhang, R., "A Study of Negative Poisson's Ratio in Randomly Oriented quasiIsotropic Composite Laminates," Journal of Composite Materials, Vol. 33, No. 19, 1999, pp. 1843-1857.

${ }^{11}$ Walker, M., Adali, S., and Verijenko, V. E., "Optimal Design of Laminated Composite Plates for Maximum Buckling Load Subject to In-Plane Restraints Using the FEM," Computer Aided Design in Composite Material Technology IV, W. R. Blain and W. P. De Wilde, eds., Computational Mechanics Publications, Southampton, UK, June 1994, pp. 119-128.

${ }^{12}$ Adali, S., Walker, M., and Verijenko, V. E., "Multiobjective Optimization of Laminated Plates for Maximum Prebuckling, Buckling and Postbuckling Strength Using Continuous and Discrete Ply Angles," Composite Structures, Vol. 35, 1996, pp. 117-130.

${ }^{13}$ Bedair, O. K., "Influence of In-Plane Restraint on the Buckling Behavior of Plates Under Compression, Shear and In-plane Bending," Computer Methods in Applied Mechanics and Engineering, Vol. 148, 1997, pp. 1-10.
${ }^{14}$ Bedair, O. K., "Effects of Lateral Restraint on the Buckling Behavior of Plates Under Non-Uniform Edge Compression," Structural Engineering and Mechanics, Vol. 5, No. 1, 1997, pp. 85-104

${ }^{15}$ Walker, M., Adali, S., and Verijenko, V. E., "Optimal Design of Symmetric Angle-Ply Laminates Subject to Nonuniform Buckling Loads and In-Plane Restraints," Thin-Walled Structures, Vol. 26, No. 1, 1996, pp. 45-60.

${ }^{16}$ Nemeth, M. P., "Buckling Behavior of Long Anisotropic Plates Subjected to Restrained Thermal Expansion and Mechanical Loads," AIAA Paper N0. 99-1229, April 1999.

${ }^{17}$ Nemeth, M. P., "Buckling Behavior of Long Anisotropic Plates Subjected to Restrained Thermal Expansion and Mechanical Loads," Journal of Thermal Stresses, Vol. 23, 2000, pp. 873-916.

${ }^{18}$ Nemeth, M. P., "Buckling Behavior of Long Anisotropic Plates Subjected to Fully Restrained Thermal Expansion," AIAA Paper No. 2001-1330, April 2001.

${ }^{19}$ Nemeth, M. P., "Buckling Behavior of Long Anisotropic Plates Subjected to Elastically Restrained Thermal Expansion," AIAA Paper No. 2002-1731, April 2002.

${ }^{20}$ Nemeth, M. P., "Importance of Anisotropy on Buckling of Compression-Loaded Symmetric Composite Plates," AIAA Journal, Vol. 24, No. 11, November 1986, pp. 1831-1835.

${ }^{21}$ Jones, R. M., Mechanics of Composite Materials, second edition, Taylor \& Francis, Inc., Philadelphia, 1999.

Table 1. Lamina Properties

\begin{tabular}{|c|c|c|c|c|c|c|c|c|c|}
\hline \multirow{2}{*}{$\begin{array}{c}\text { Lamina } \\
\text { property* }\end{array}$} & \multicolumn{9}{|c|}{ Material Systems } \\
\cline { 2 - 11 } & $\begin{array}{c}\text { Boron } \\
-\mathrm{Al}\end{array}$ & $\begin{array}{c}\text { S-glass- } \\
\text { epoxy }\end{array}$ & $\begin{array}{c}\text { Kevlar 49- } \\
\text { epoxy }\end{array}$ & $\begin{array}{c}\text { IM7/ } \\
5260\end{array}$ & $\begin{array}{c}\text { AS4/ } \\
3502\end{array}$ & $\begin{array}{c}\text { AS4/ } \\
3501-6\end{array}$ & $\begin{array}{c}\text { Boron- } \\
\text { epoxy }\end{array}$ & $\begin{array}{c}\text { IM7/ } \\
\text { PETI-5 }\end{array}$ & $\begin{array}{c}\text { P-100/ } \\
3502\end{array}$ \\
\hline $\mathrm{E}_{\mathrm{L}}, \mathrm{Msi}$ & 33 & 7.5 & 11.02 & 22.1 & 18.5 & 20.01 & 29.58 & 20.35 & 53.5 \\
\hline $\mathrm{E}_{\mathrm{T}}, \mathrm{Msi}$ & 21 & 1.7 & 0.8 & 1.457 & 1.64 & 1.30 & 2.68 & 1.16 & 0.73 \\
\hline $\mathrm{v}_{\mathrm{LT}}$ & 0.23 & 0.25 & 0.34 & 0.258 & 0.30 & 0.30 & 0.23 & 0.29 & 0.31 \\
\hline $\mathrm{G}_{\mathrm{LT}}, \mathrm{Msi}$ & 7.0 & 0.80 & 0.33 & 0.860 & 0.87 & 1.03 & 0.81 & 0.61 & 0.76 \\
\hline$\rho, 1 \mathrm{~b} / \mathrm{in}^{3}$ & 0.098 & 0.072 & 0.050 & 0.056 & 0.059 & 0.056 & 0.075 & 0.058 & 0.062 \\
\hline
\end{tabular}

* The symbols L and $\mathrm{T}$ denote the longitudinal fiber and transverse matrix directions of a specially orthotropic lamina, respectively. 
Table 2. Load ratio $\left(1+R_{2}\right) \frac{N_{y 1}}{N_{x 1}^{c}}$ for $\left[( \pm 45 / 0 / 90)_{m}\right]_{s},\left[\left( \pm 45 / 0_{2}\right)_{m}\right]_{s}$, and $\left.\left[( \pm 45 / 90)_{2}\right]_{\mathrm{s}}\right]_{s}$ laminates $(m=1,2, \ldots)$.

\begin{tabular}{|c|c|c|c|}
\hline \multirow{2}{*}{ Material Systems } & \multicolumn{3}{|c|}{$\left(1+\mathrm{R}_{2}\right)^{*} \mathrm{~N}_{\mathrm{y} 1}^{\mathrm{N}} \mathrm{N}_{\mathrm{x} 1}$} \\
\cline { 2 - 4 } & {$\left[( \pm 45 / 0 / 90)_{\mathrm{m}}\right]_{\mathrm{s}}$} & {$\left[\left( \pm 45 / 0_{2}\right)_{\mathrm{m}}\right]_{\mathrm{s}}$} & {$\left[\left( \pm 45 / 90_{2}\right)_{\mathrm{m}}\right]_{\mathrm{s}}$} \\
\hline IM7/5260 & 0.299 & 0.193 & 0.667 \\
\hline Boron-A1 & 0.281 & 0.251 & 0.320 \\
\hline S-glass-epoxy & 0.272 & 0.199 & 0.430 \\
\hline Kevlar 49-epoxy & 0.325 & 0.210 & 0.720 \\
\hline AS4/3502 & 0.303 & 0.199 & 0.631 \\
\hline AS4/3501-6 & 0.284 & 0.184 & 0.623 \\
\hline Boron-epoxy & 0.323 & 0.211 & 0.690 \\
\hline IM7/PETI-5 & 0.312 & 0.199 & 0.721 \\
\hline P-100/3502 & 0.316 & 0.193 & 0.867 \\
\hline
\end{tabular}

Table 3. Load ratio $\left(1+R_{2}\right) \frac{N_{y 1}}{N_{x 1}^{c}}$ for $\left[\left(+45_{2} / 0 / 90\right)_{m}\right]_{s},\left[\left(+45_{2} / 0_{2}\right)_{m}\right]_{s}$, and $\left[\left(+45_{2} / 90_{2}\right)_{m}\right]_{s}$ laminates $\left(R_{3} \rightarrow \infty, m=1,2, \ldots\right)$.

\begin{tabular}{|c|c|c|c|}
\hline \multirow{2}{*}{ Material Systems } & \multicolumn{3}{|c|}{$\left(1+\mathrm{R}_{2}\right)^{-} \mathrm{N}_{\mathrm{y} 1} \mathrm{~N}_{\mathrm{x} 1}^{\mathrm{c}}=\mathrm{v}_{\mathrm{yx}}$} \\
\cline { 2 - 4 } & {$\left[\left(+45_{2} / 0 / 90\right)_{\mathrm{m}}\right]_{\mathrm{s}}$} & {$\left[\left(+45_{2} / 0_{2}\right)_{\mathrm{m}}\right]_{\mathrm{s}}$} & {$\left[\left(+45_{2} / 90_{2}\right)_{\mathrm{m}}\right]_{\mathrm{s}}$} \\
\hline IM7/5260 & 0.105 & 0.062 & 0.355 \\
\hline Boron-Al & 0.274 & 0.244 & 0.312 \\
\hline S-glass-epoxy & 0.198 & 0.141 & 0.332 \\
\hline Kevlar 49-epoxy & 0.133 & 0.078 & 0.448 \\
\hline AS4/3502 & 0.135 & 0.082 & 0.381 \\
\hline AS4/3501-6 & 0.098 & 0.058 & 0.311 \\
\hline Boron-epoxy & 0.145 & 0.087 & 0.441 \\
\hline IM7/PETI-5 & 0.102 & 0.059 & 0.393 \\
\hline P-100/3502 & 0.030 & 0.016 & 0.304 \\
\hline
\end{tabular}


Table 4. Load ratio $\left(1+R_{3}\right) \frac{N_{x y 1}}{N_{x 1}^{c}}$ for $\left[\left(+45_{2} / 0 / 90\right)_{m}\right]_{s},\left[\left(+45_{2} / 0_{2}\right)_{m}\right]_{s}$, and $\left[\left(+45_{2} / 90_{2}\right)_{m}\right]_{s}$ laminates $\left(R_{2} \rightarrow \infty, m=1,2, \ldots\right)$.

\begin{tabular}{|c|c|c|c|}
\hline \multirow{2}{*}{ Material Systems } & \multicolumn{3}{|c|}{$\left(1+\mathrm{R}_{3}\right) \frac{\mathrm{N}_{\mathrm{xy} 1}}{\mathrm{~N}_{\mathrm{x} 1}^{\mathrm{c}}}=\eta_{\mathrm{x}, \mathrm{xy}}$} \\
\cline { 2 - 4 } & {$\left[\left(+45_{2} / 0 / 90\right)_{\mathrm{m}}\right]_{\mathrm{s}}$} & {$\left[\left(+45_{2} / 0_{2}\right)_{\mathrm{m}}\right]_{\mathrm{s}}$} & {$\left[\left(+45_{2} / 90_{2}\right)_{\mathrm{m}}\right]_{\mathrm{s}}$} \\
\hline IM7/5260 & -0.212 & -0.068 & -0.570 \\
\hline Boron-A1 & -0.047 & -0.040 & -0.056 \\
\hline S-glass-epoxy & -0.144 & -0.084 & -0.254 \\
\hline Kevlar 49-epoxy & -0.207 & -0.059 & -0.564 \\
\hline AS4/3502 & -0.200 & -0.072 & -0.496 \\
\hline AS4/3501-6 & -0.212 & -0.075 & -0.549 \\
\hline Boron-epoxy & -0.201 & -0.063 & -0.524 \\
\hline IM7/PETI-5 & -0.216 & -0.059 & -0.614 \\
\hline P-100/3502 & -0.241 & -0.031 & -0.843 \\
\hline
\end{tabular}

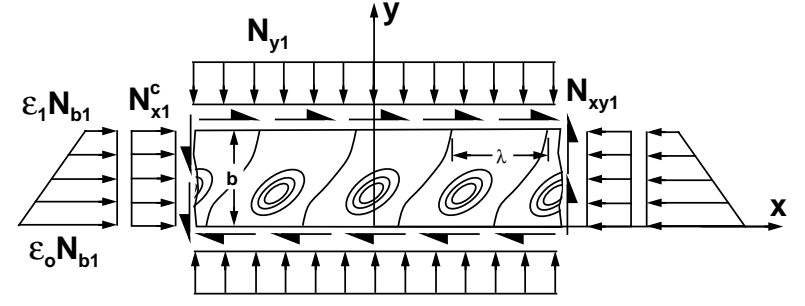

(a) Destabilizing loading system

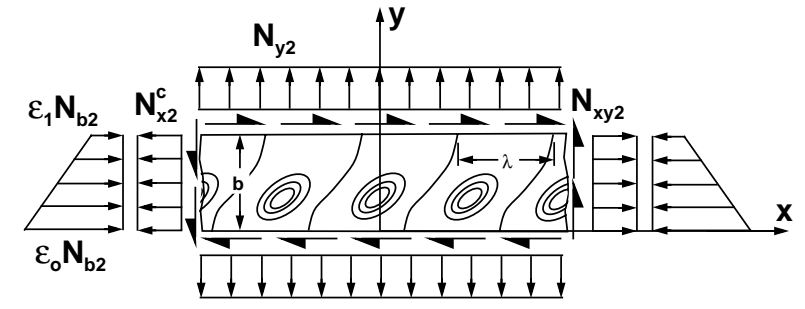

(b) Subcritical loading system

Fig. 1 Sign convention for positive-valued stress resultants.

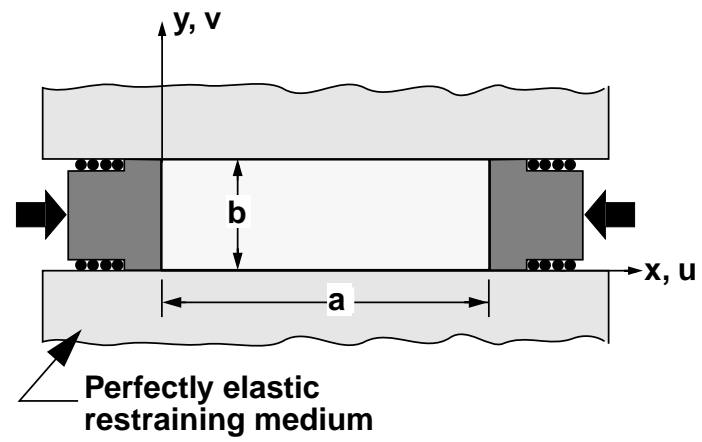

(a) Boundary conditions

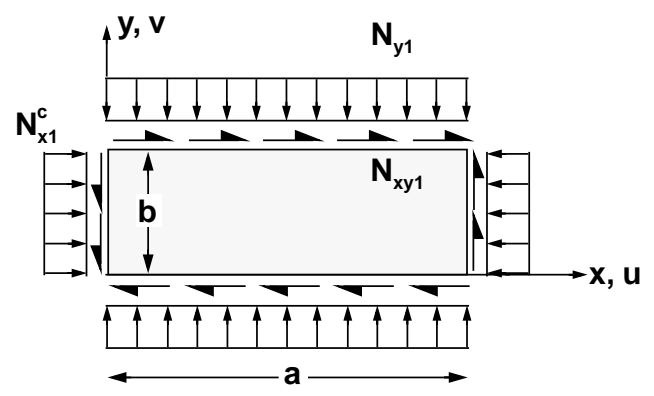

(b) Positive values for induced loads

Fig. 2 Mechanical loads in a compression-loaded plate elastically restrained against lateral expansion or contraction and inplane shearing deformations. 


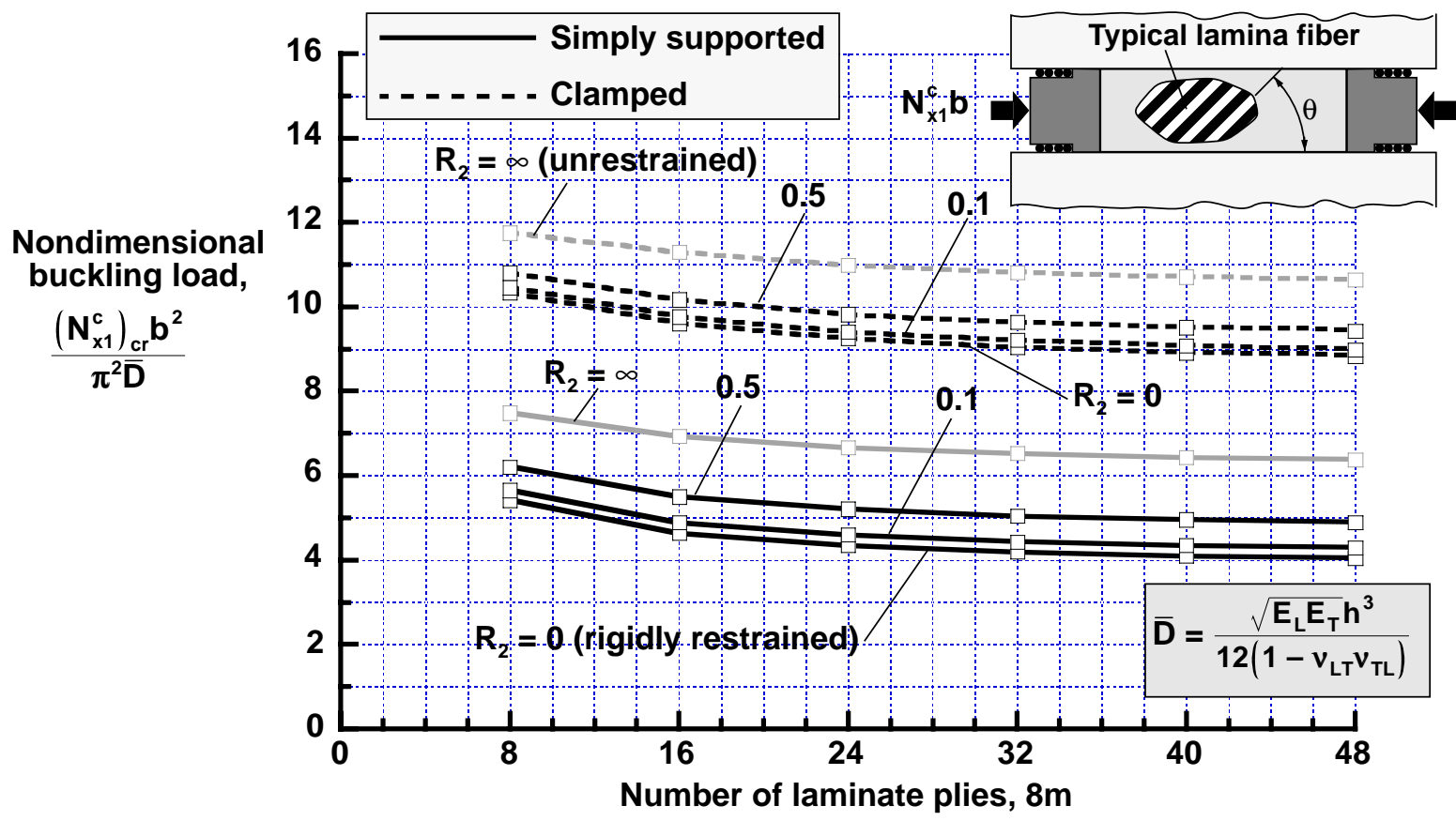

Fig. 3 Effects of compliance ratio $R_{2}$ on nondimensional buckling load for $\left[\left( \pm 45 / 0_{2}\right)_{\mathrm{m}}\right]_{\mathrm{s}}$ balanced laminates made of IM7/5260 material.

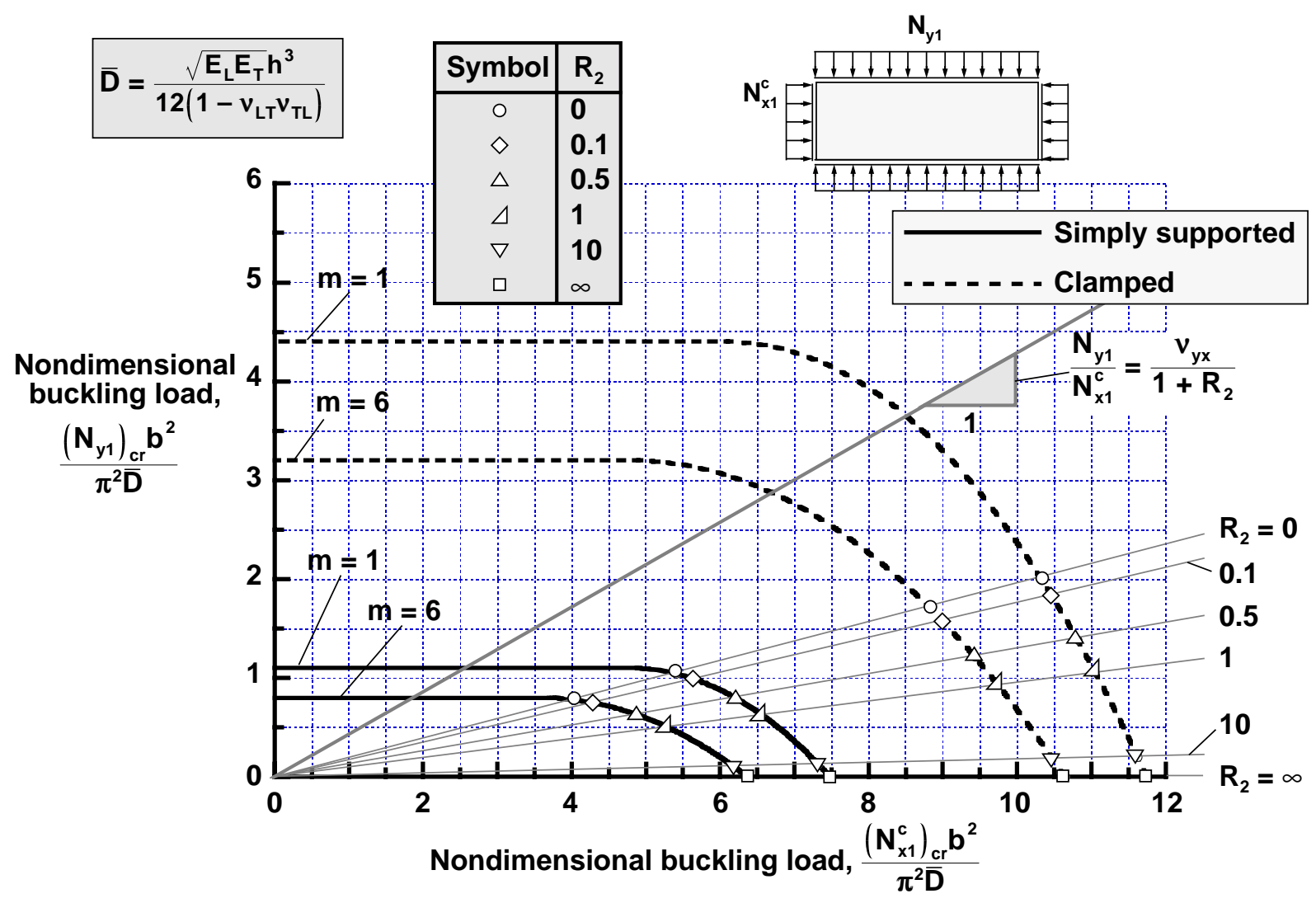

Fig. 4 Nondimensional buckling interaction curves for clamped and simply supported $\left[\left( \pm 45 / 0_{2}\right)_{\mathrm{m}}\right]_{\mathrm{s}}$ balanced laminates made of IM7/5260 material and subjected to biaxial compression $(m=1,6)$. 


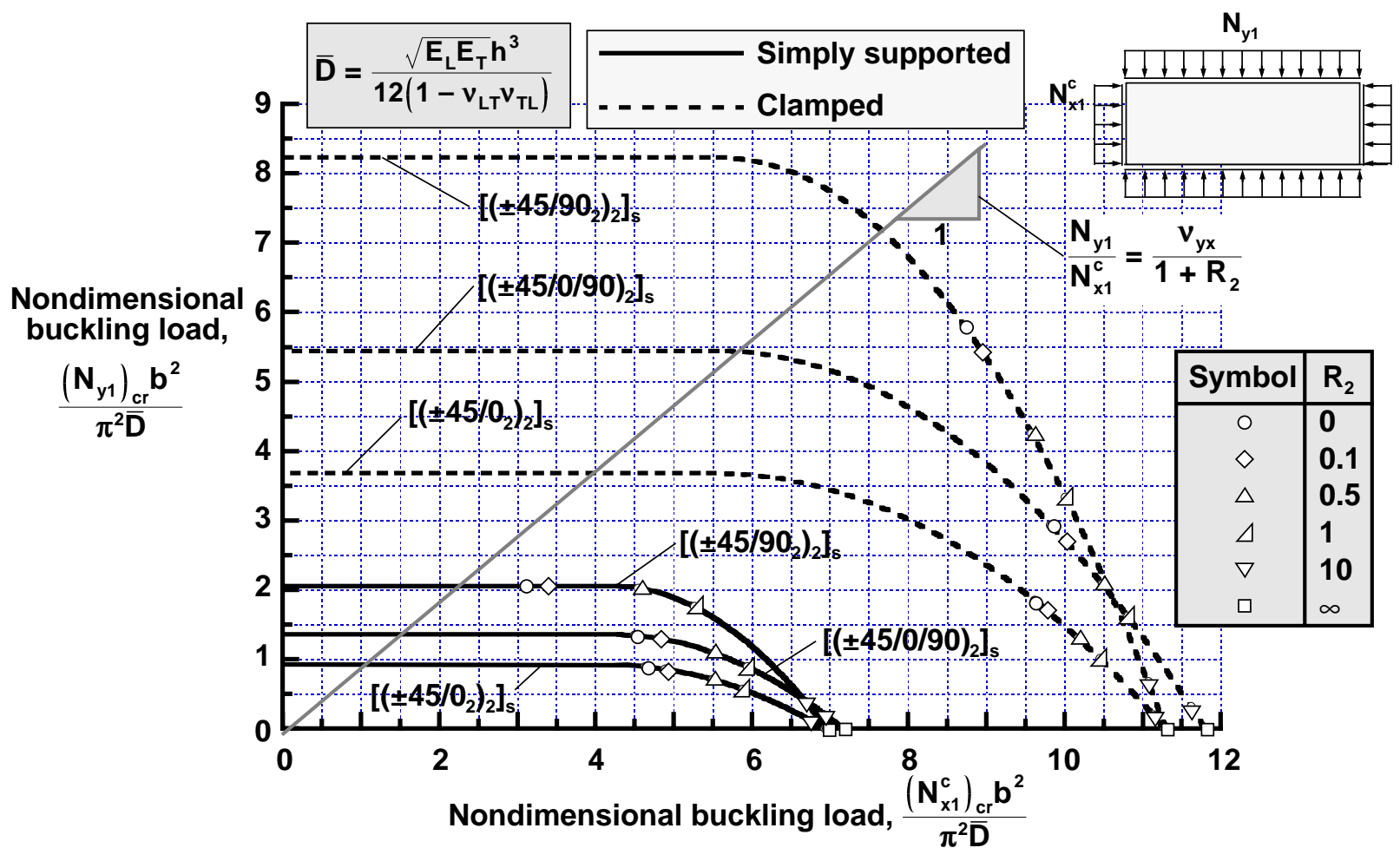

Fig. 5 Nondimensional buckling interaction curves for clamped and simply supported $\left[\left( \pm 45 / 0_{2}\right)_{2}\right]_{s},\left[( \pm 45 / 0 / 90)_{2}\right]_{\text {s }}$, and $\left[( \pm 45 / 90)_{2}\right]_{\mathrm{s}}$ balanced laminates made of IM7/5260 material and subjected to biaxial compression.

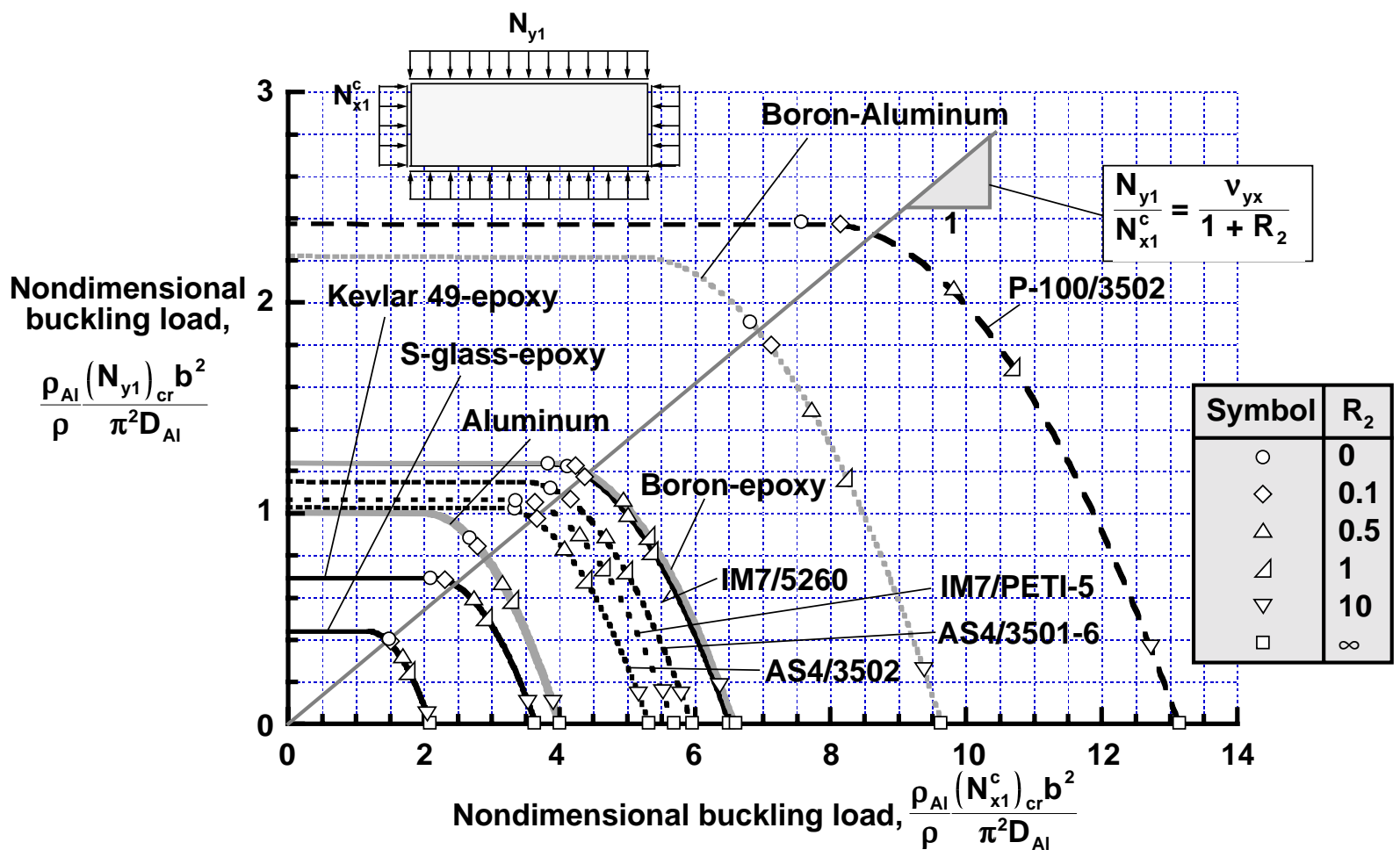

Fig. 6 Nondimensional buckling interaction curves for simply supported $\left[( \pm 45 / 0 / 90)_{2}\right]_{\mathrm{s}}$ balanced laminates subjected to biaxial compression, for ten different material systems. 


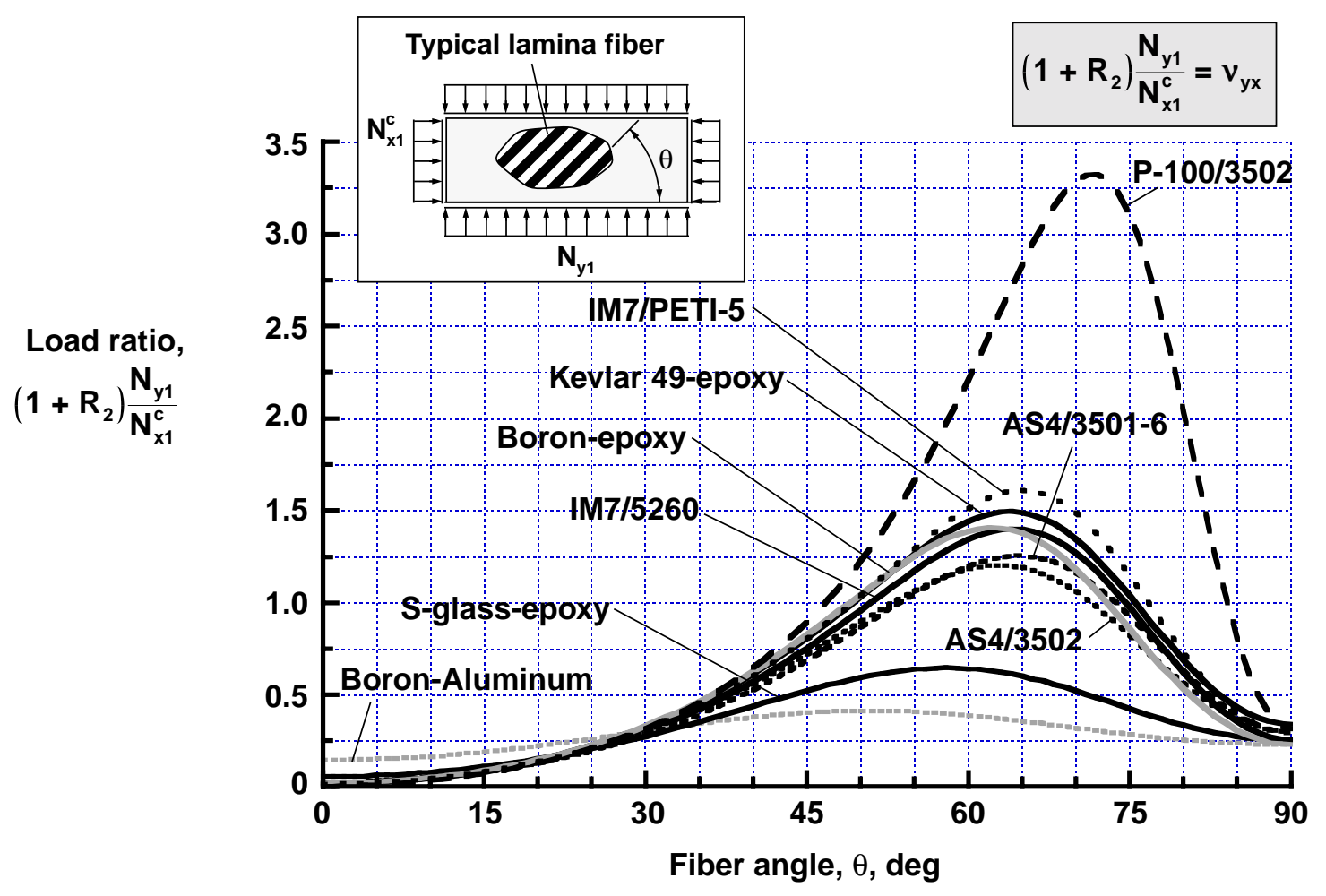

Fig. 7 Effects of lamina material properties on load ratio $\left(1+\mathbf{R}_{2}\right) \mathbf{N}_{\mathrm{y} 1} / \mathbf{N}_{\mathrm{x} 1}^{\mathrm{c}}$ for $\left[( \pm \theta)_{\mathrm{m}}\right]_{\mathrm{s}}$ balanced, angle-ply laminates $(m=1,2,3, \ldots)$.

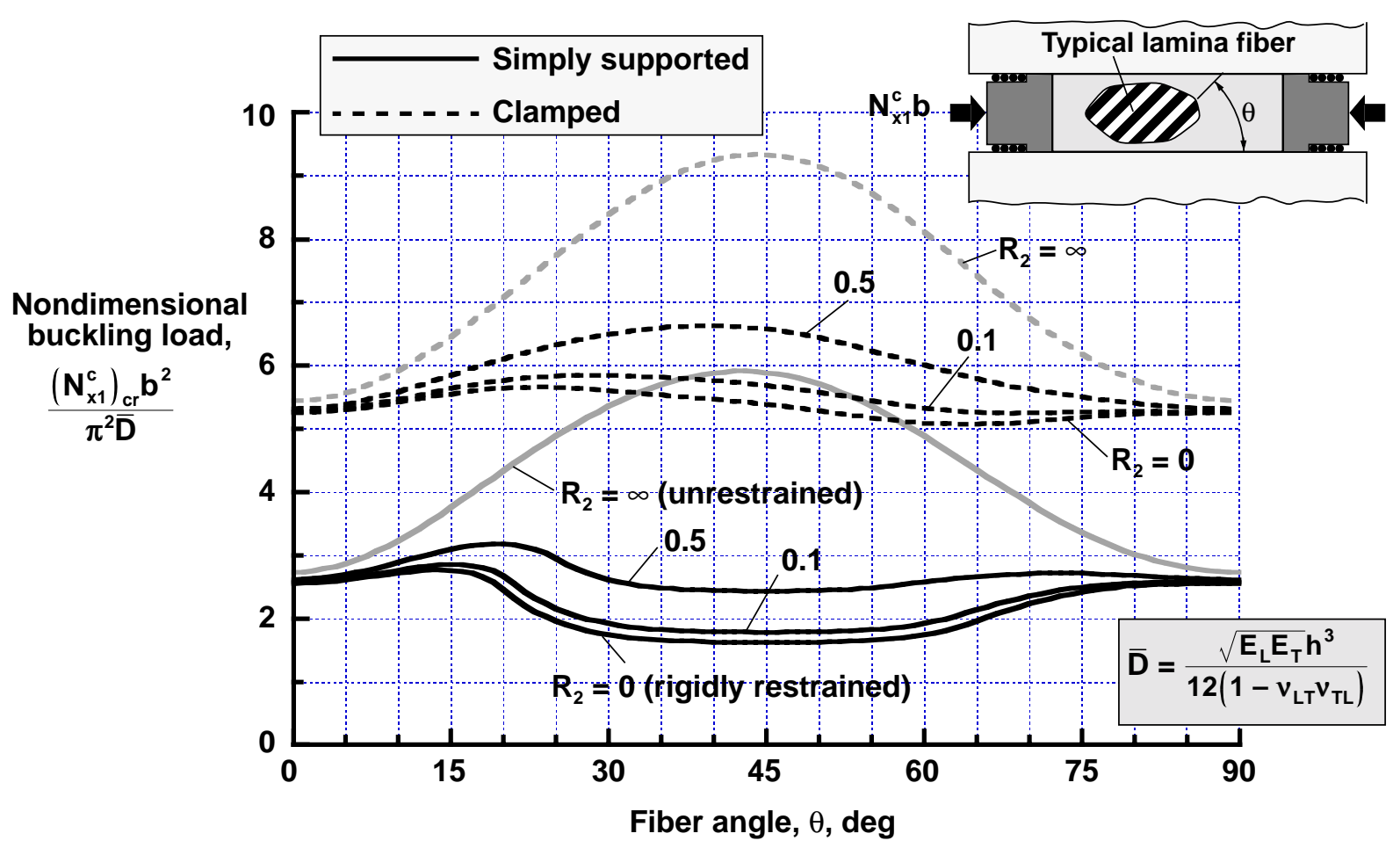

Fig. 8 Effects of compliance ratio $R_{2}$ on nondimensional buckling load for $[ \pm \theta]_{s}$ balanced, angle-ply laminates made of IM7/5260 material. 


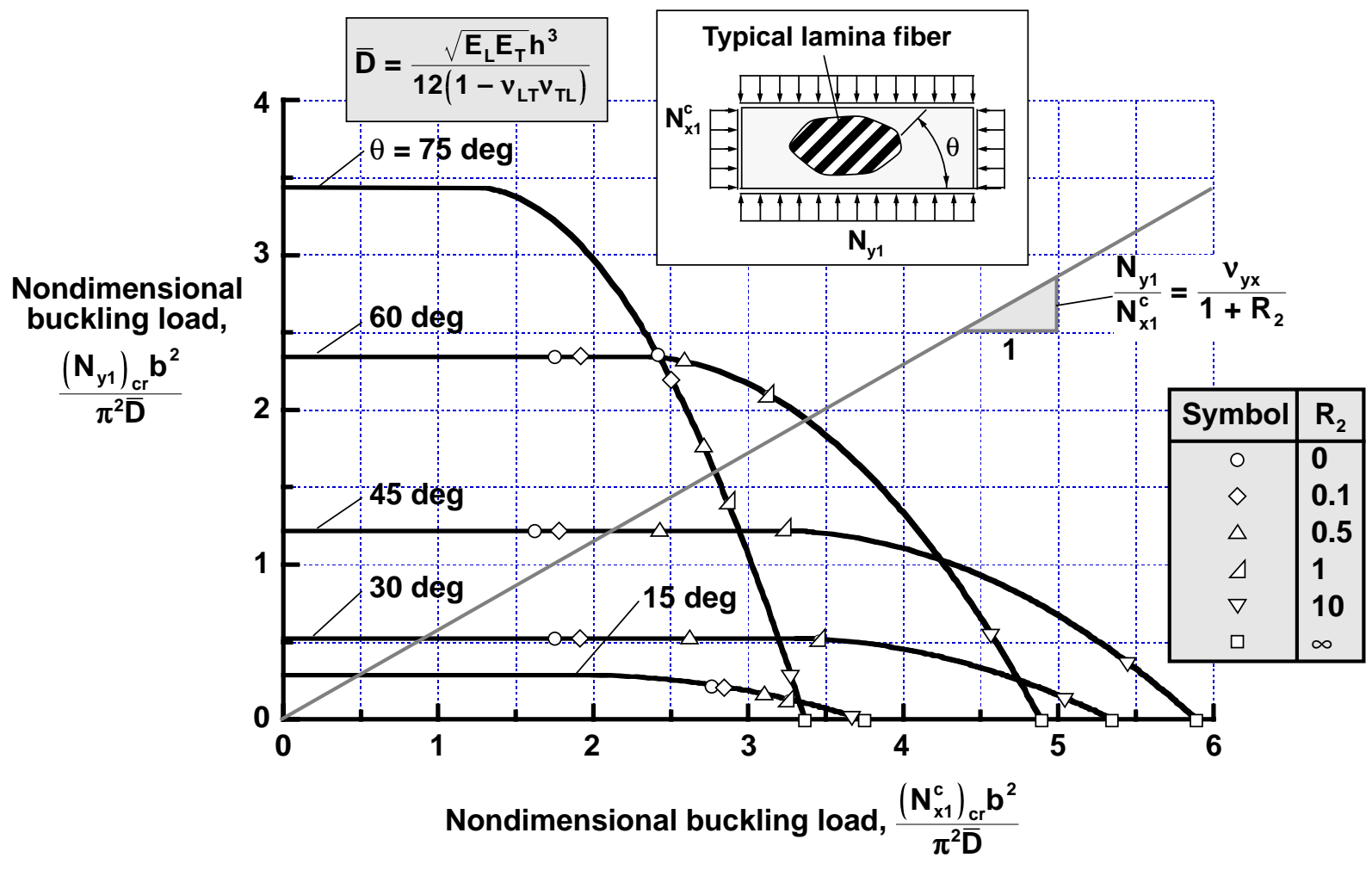

Fig. 9 Nondimensional buckling interaction curves for simply supported $[ \pm \theta]_{\mathrm{s}}$ balanced, angle-ply laminates made of IM7/5260 material and subjected to biaxial compression. 


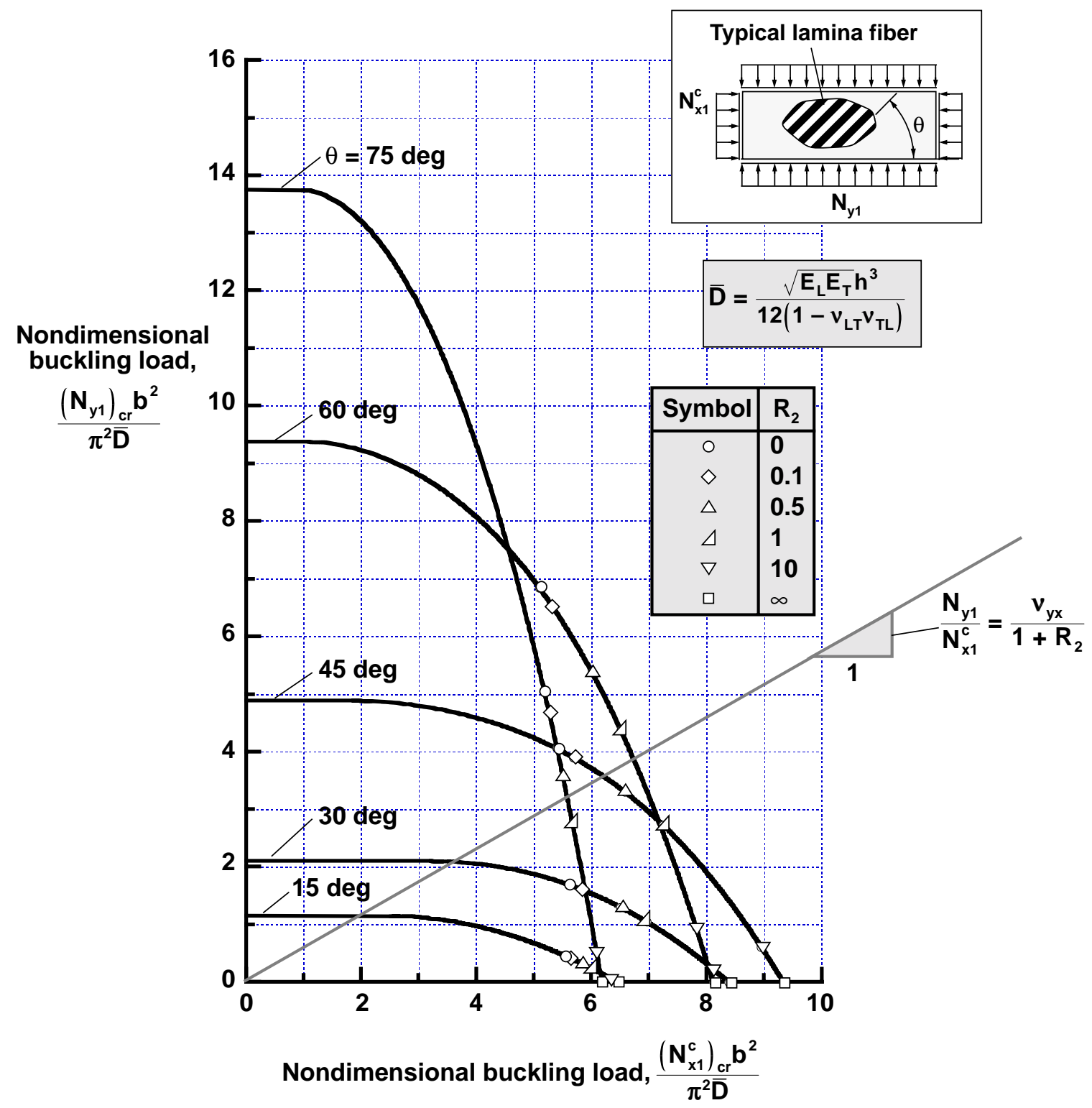

Fig. 10 Nondimensional buckling interaction curves for clamped $[ \pm \theta]_{\mathrm{s}}$ balanced, angle-ply laminates made of IM7/5260 material and subjected to biaxial compression. 


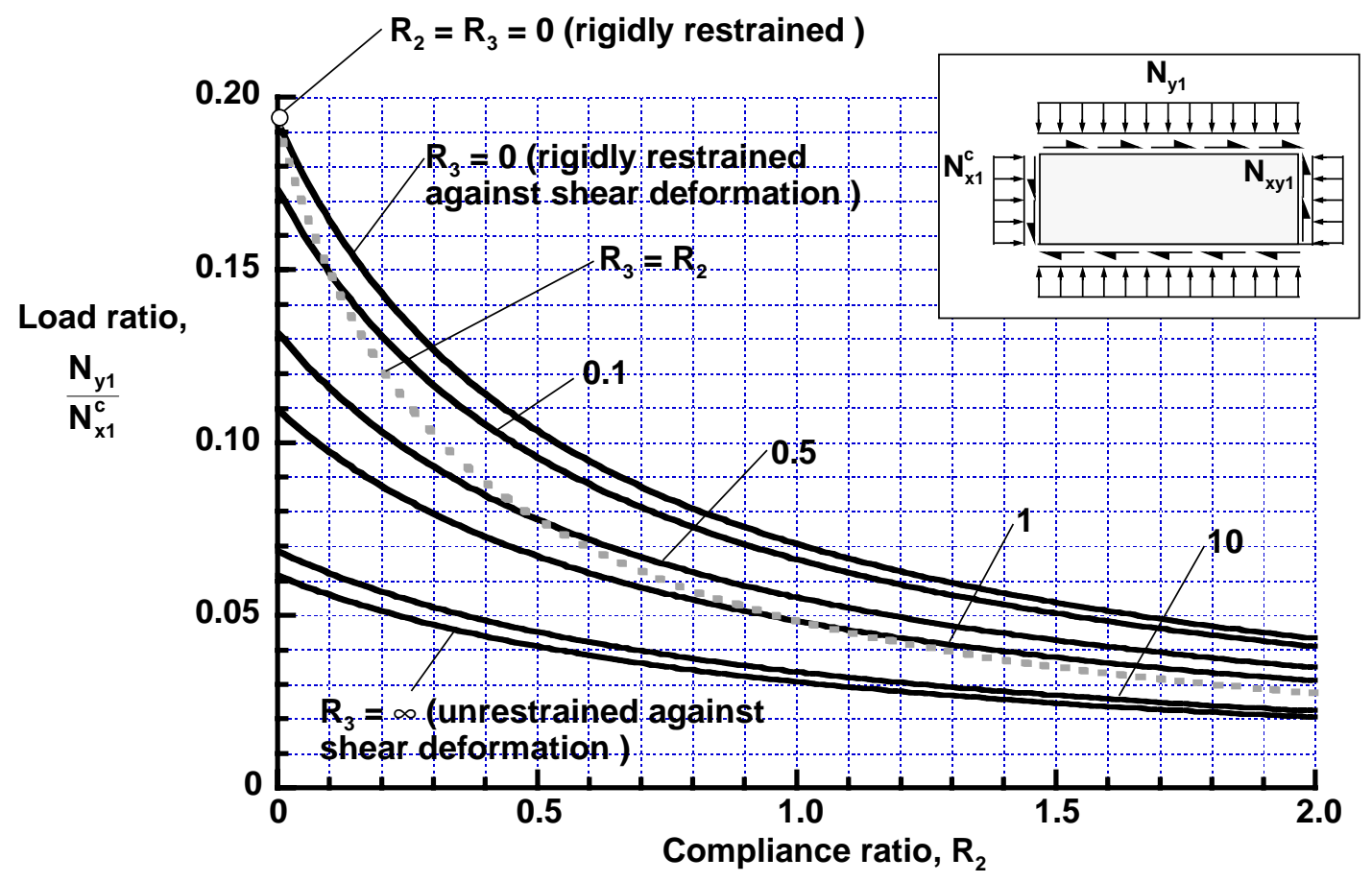

Fig. 11 Effects of compliance ratios $R_{2}$ and $R_{3}$ on nondimensional load ratio $N_{y 1} / N_{x 1}^{c}$ for $\left[\left(+45_{2} / 0_{2}\right)_{m}\right]_{s}$ unbalanced laminates made of $\mathrm{IM} 7 / 5260$ material $(\mathrm{m}=1,2, \ldots)$.

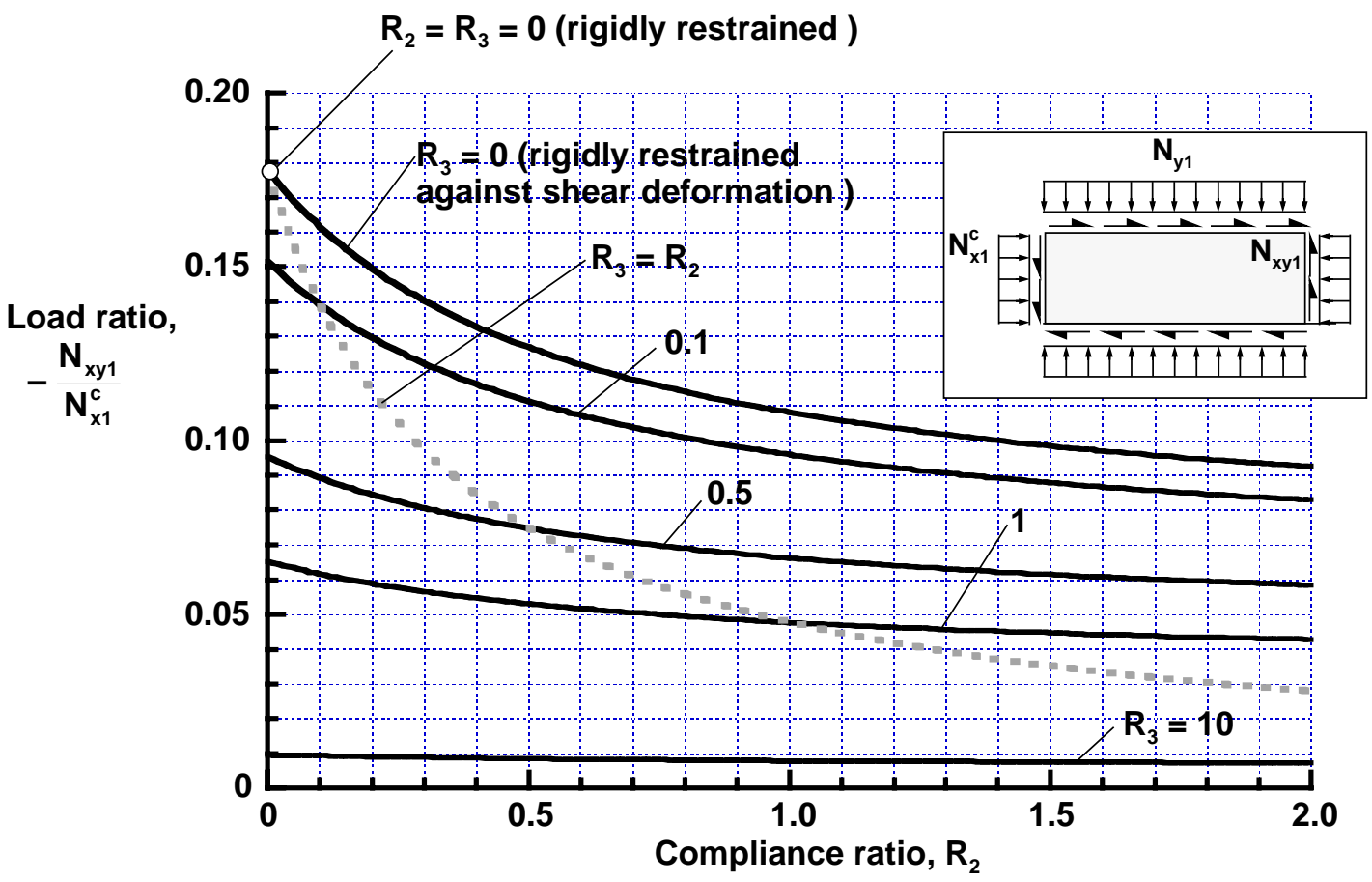

Fig. 12 Effects of compliance ratios $R_{2}$ and $R_{3}$ on nondimensional load ratio $N_{x y 1} / N_{x 1}^{c}$ for $\left[\left(+45_{2} / 0_{2}\right)_{m}\right]_{s}$ unbalanced laminates made of $\mathrm{IM} 7 / 5260$ material $(\mathrm{m}=1,2, \ldots)$. 


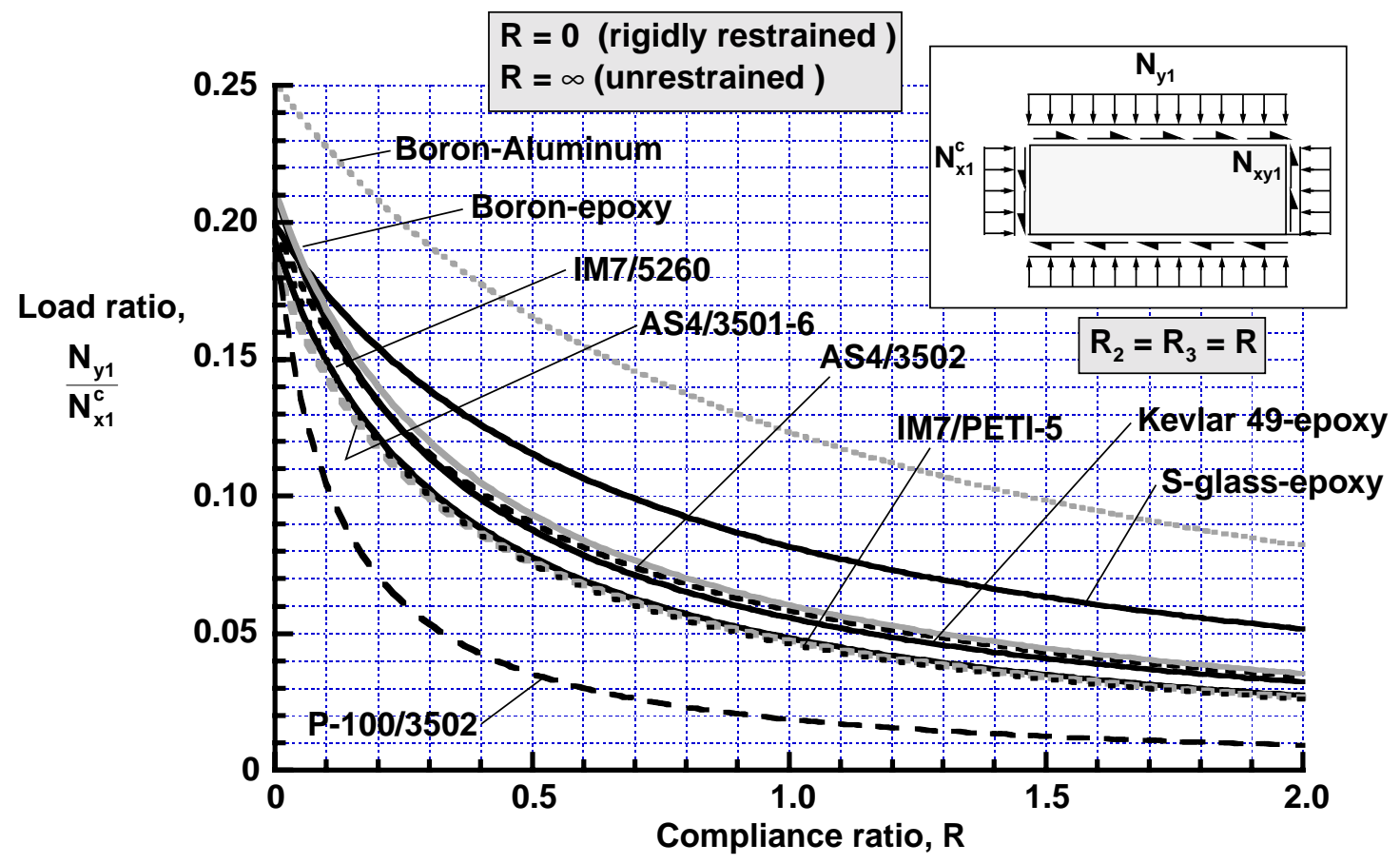

Fig. 13 Effects of compliance ratio $R=R_{2}=R_{3}$ on nondimensional load ratio $N_{y 1} / N_{x 1}^{c}$ for $\left[\left(+45_{2} / 0_{2}\right)_{m}\right]_{s}$ unbalanced laminates made of $\mathrm{IM} 7 / 5260$ material $(\mathrm{m}=1,2, \ldots)$.

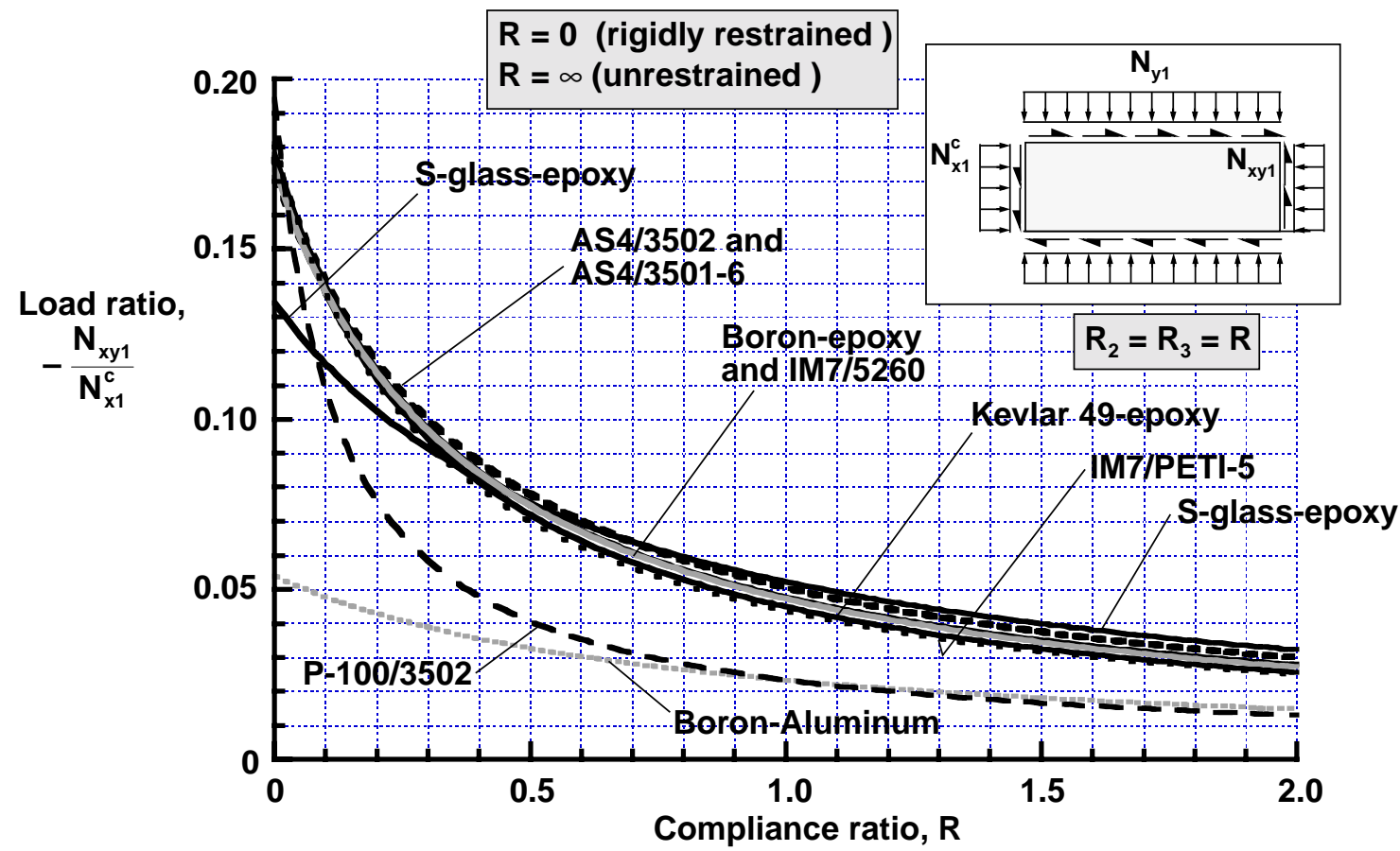

Fig. 14 Effects of compliance ratio $R=R_{2}=R_{3}$ on nondimensional load ratio $N_{x y 1} / N_{x 1}^{c}$ for $\left[\left(+45_{2} / 0_{2}\right)_{m}\right]_{s}$ unbalanced laminates made of $\mathrm{IM} 7 / 5260$ material $(\mathrm{m}=1,2, \ldots)$. 


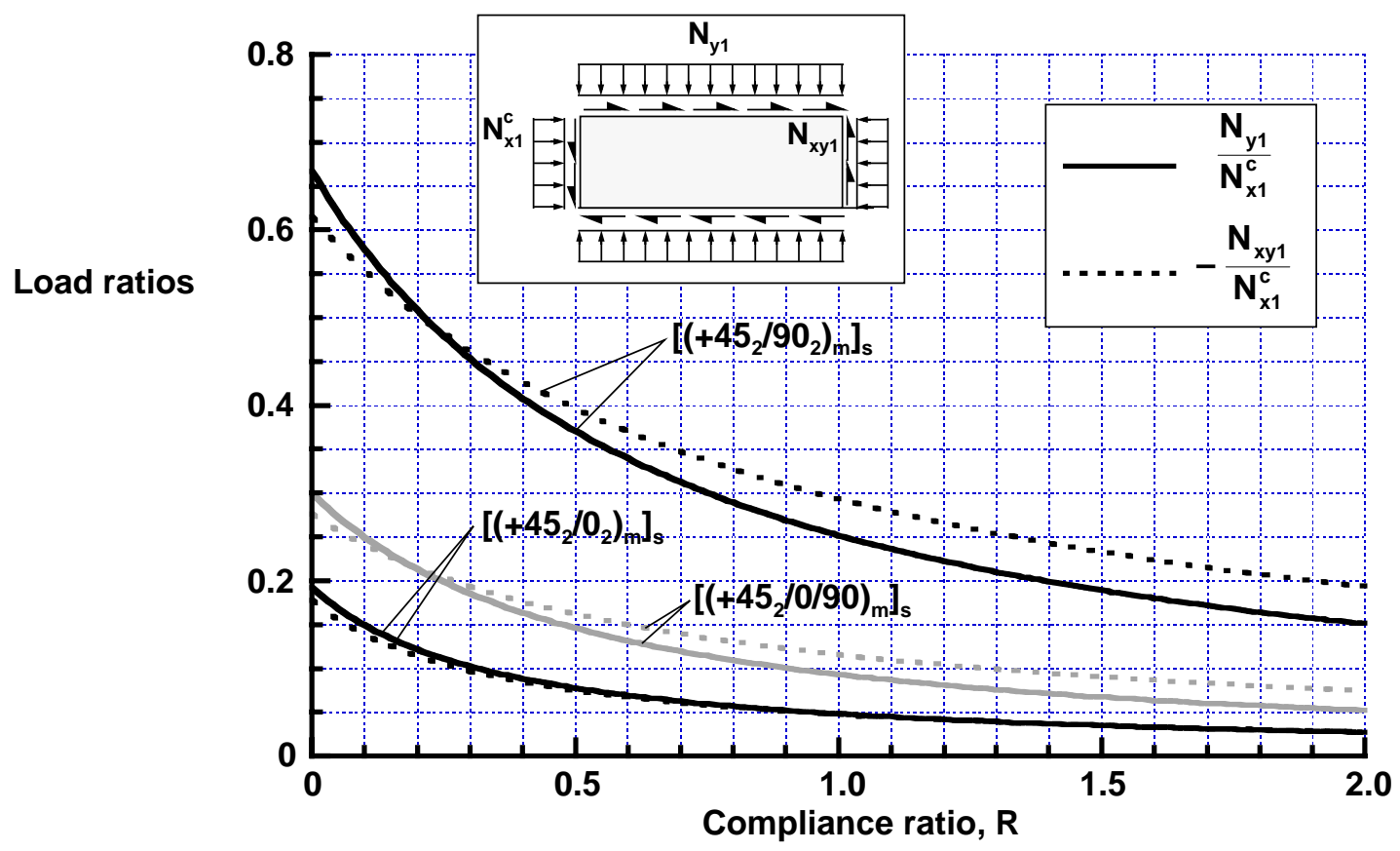

Fig. 15 Effects of compliance ratio $R$ on load ratios $N_{v 1} / N_{x 1}^{c}$ and $N_{x y 1} / N_{x 1}^{c}$ for $\left[\left(+45_{2} / 0_{2}\right)_{m}\right]_{s},\left[\left(+45_{2} / 90_{2}\right)_{m}\right]_{s}$, and $\left[\left(+45_{2} / 0 / 90\right)_{m}\right]_{s}$ unbalanced laminates made of $\operatorname{IM} 7 / 5260$ material $(\mathrm{m}=1,2, \ldots)$.

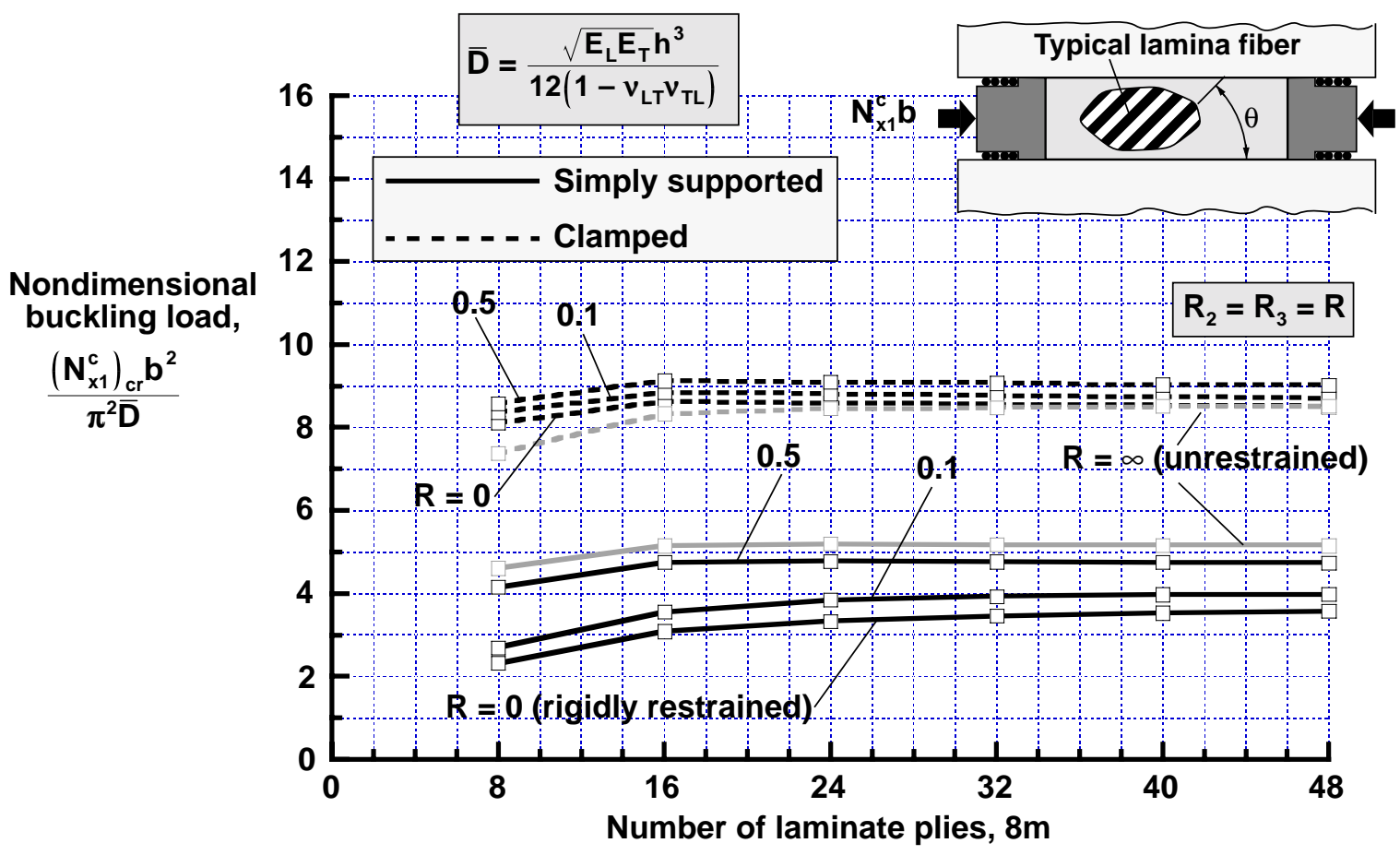

Fig. 16 Effects of compliance ratio $R$ on nondimensional buckling load for $\left[\left(+45_{2} / 90_{2}\right)_{m}\right]_{s}$ unbalanced laminates made of IM7/5260 material. 


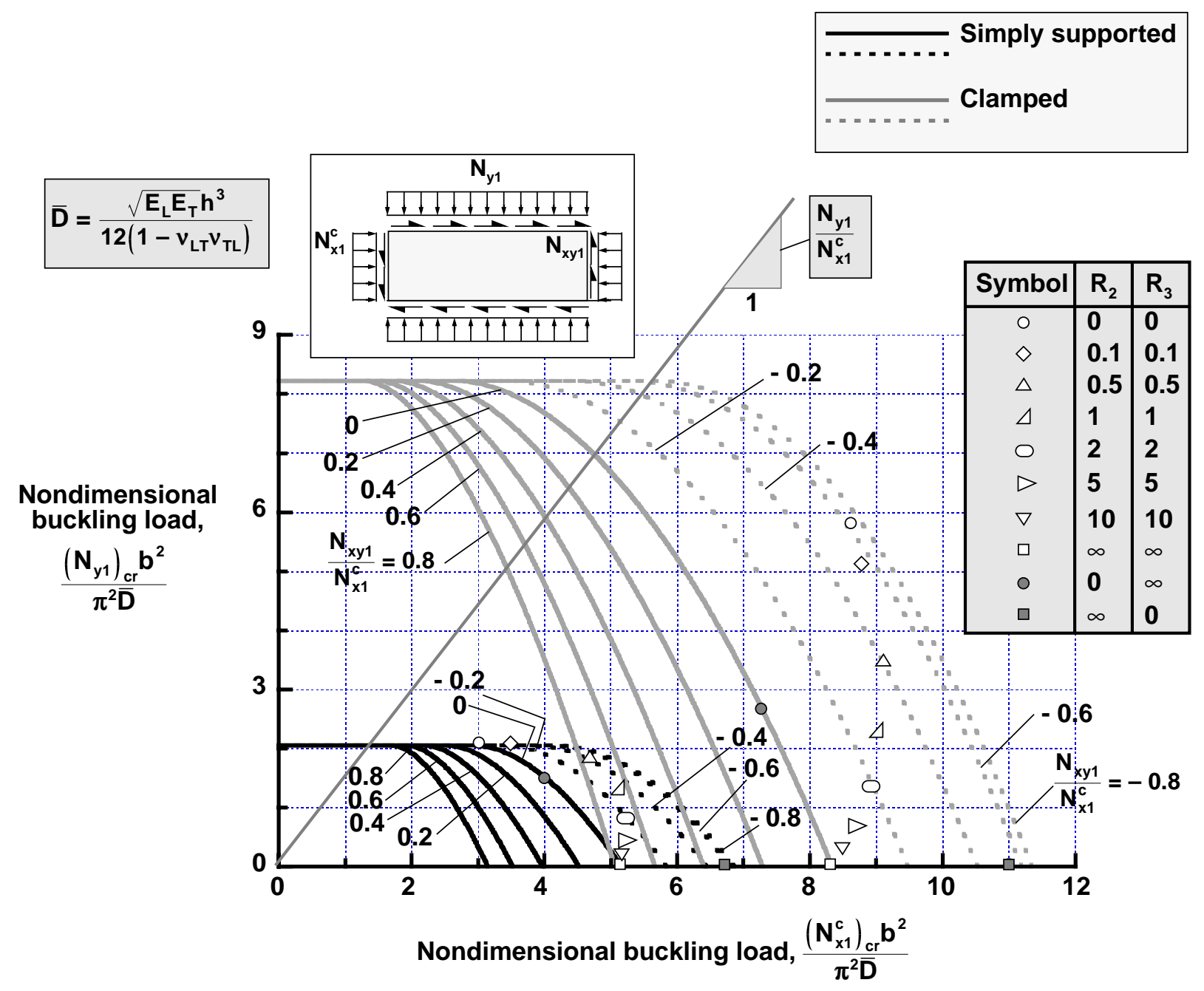

Fig. 17 Nondimensional buckling interaction curves for simply supported and clamped $\left[\left(+45_{2} / 90_{2}\right)_{2}\right]_{\mathrm{s}}$ unbalanced laminates made of IM7/5260 material and subjected to biaxial compression and shear. 


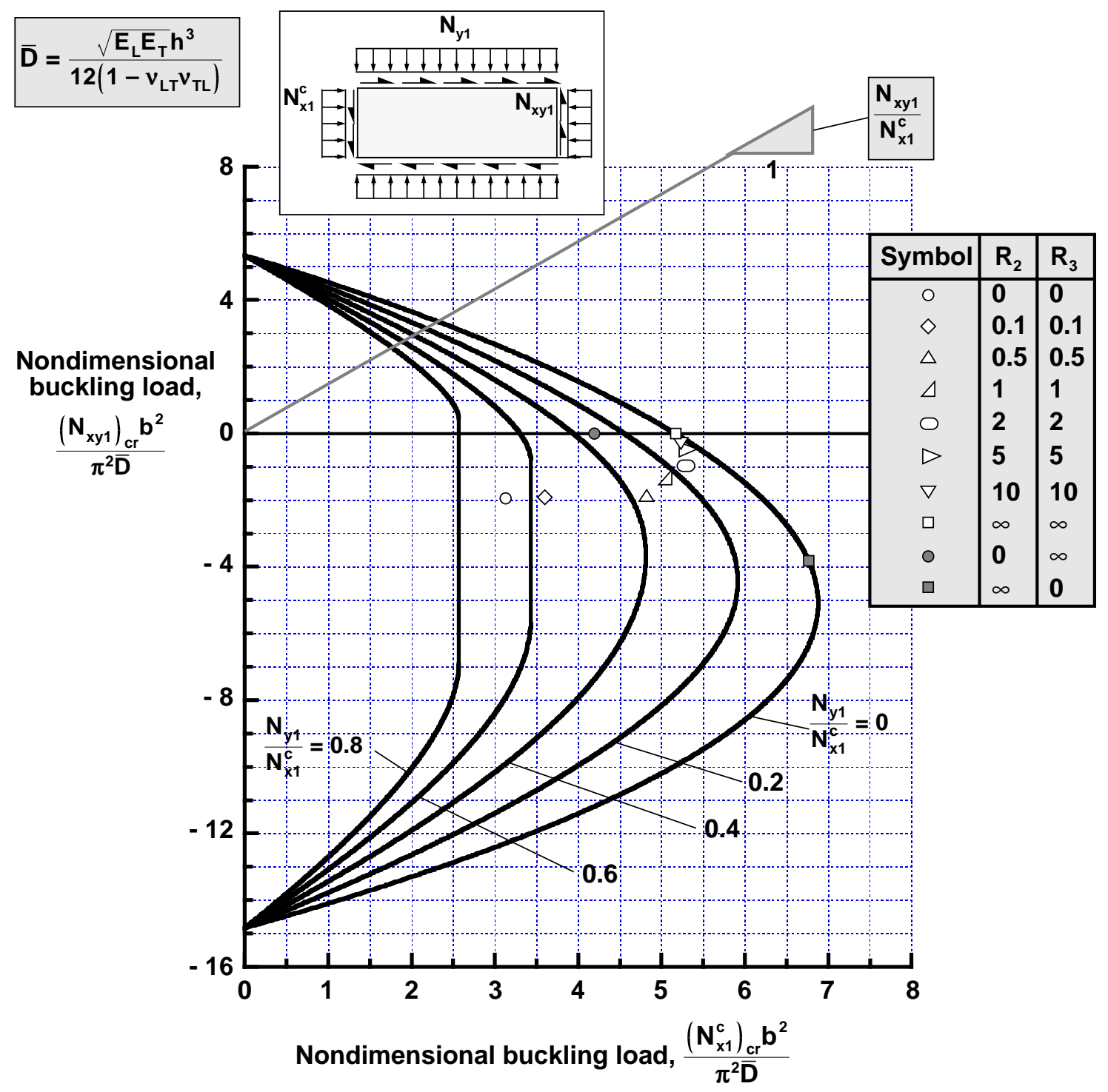

Fig. 18 Nondimensional buckling interaction curves for simply supported $\left[\left(+45_{2} / 90_{2}\right)_{2}\right]_{\mathrm{s}}$ unbalanced laminates made of IM7/5260 material and subjected to biaxial compression and shear. 


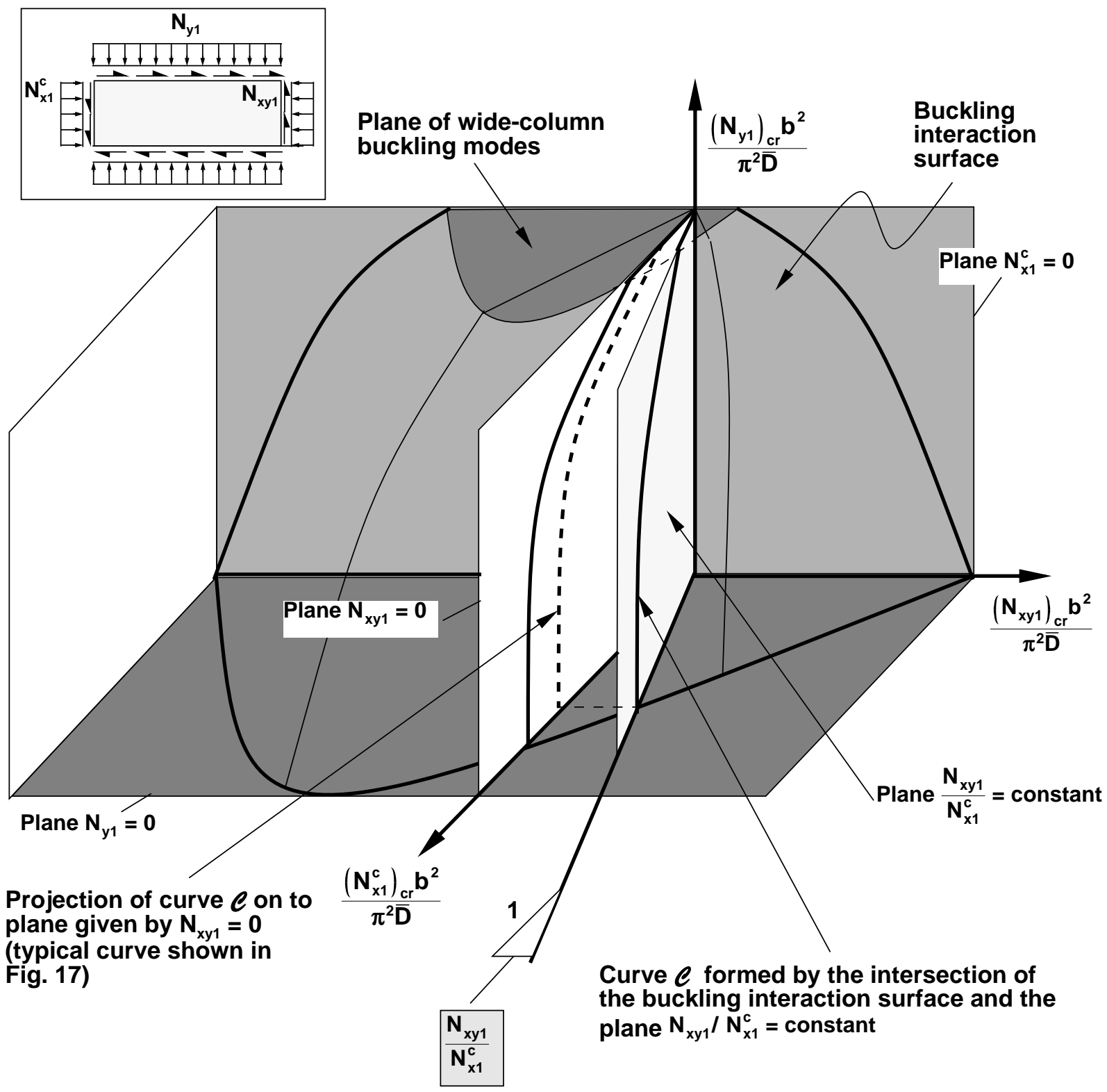

Fig. 19 Nondimensional buckling interaction surfaces and $N_{y 1}-N_{x 1}^{c}$ interaction curves for $\left[\left(+45_{2} / 90\right)_{2}\right]_{s}$ laminates made of IM7/5260 material and subjected to biaxial compression and shear. 

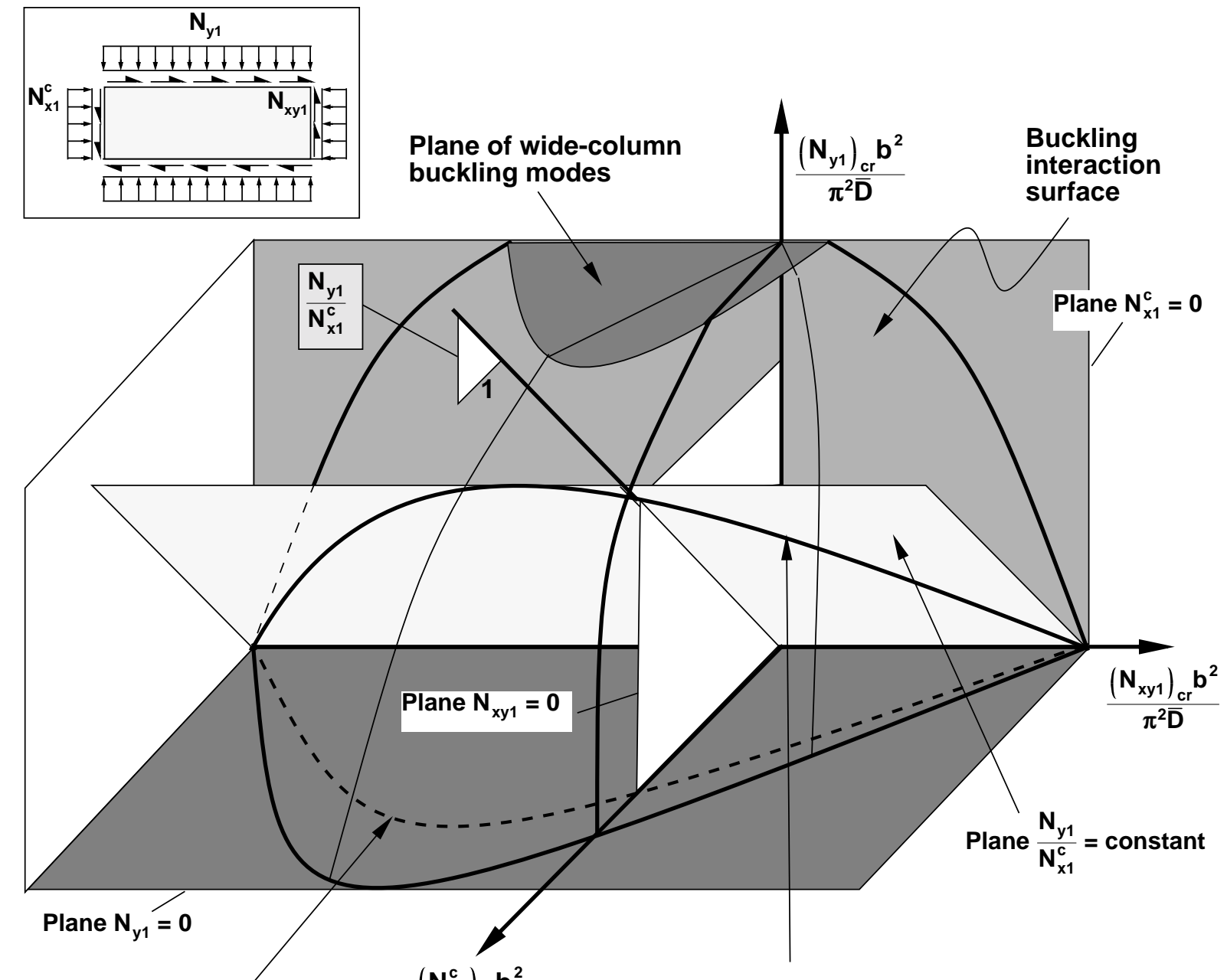

Projection of curve $e$ on to plane given by $\mathrm{N}_{\mathrm{y} 1}=0$ $\frac{\left(\mathbf{N}_{\mathrm{x} 1}^{\mathrm{c}}\right)_{\mathrm{cr}} \mathbf{b}^{2}}{\pi^{2} \overline{\mathbf{D}}}$ Curve $e$ formed by the intersection of (typical curve shown in the buckling interaction surface and the plane $\mathbf{N}_{\mathrm{y} 1} / \mathbf{N}_{\mathrm{x} 1}^{\mathrm{c}}=$ constant

Fig. 18)

Fig. 20 Nondimensional buckling interaction surfaces and $N_{x y 1}-N_{x 1}^{c}$ interaction curves for $\left[\left(+45_{2} / 90_{2}\right)_{2}\right]_{s}$ laminates made of IM7/5260 material and subjected to biaxial compression and shear. 


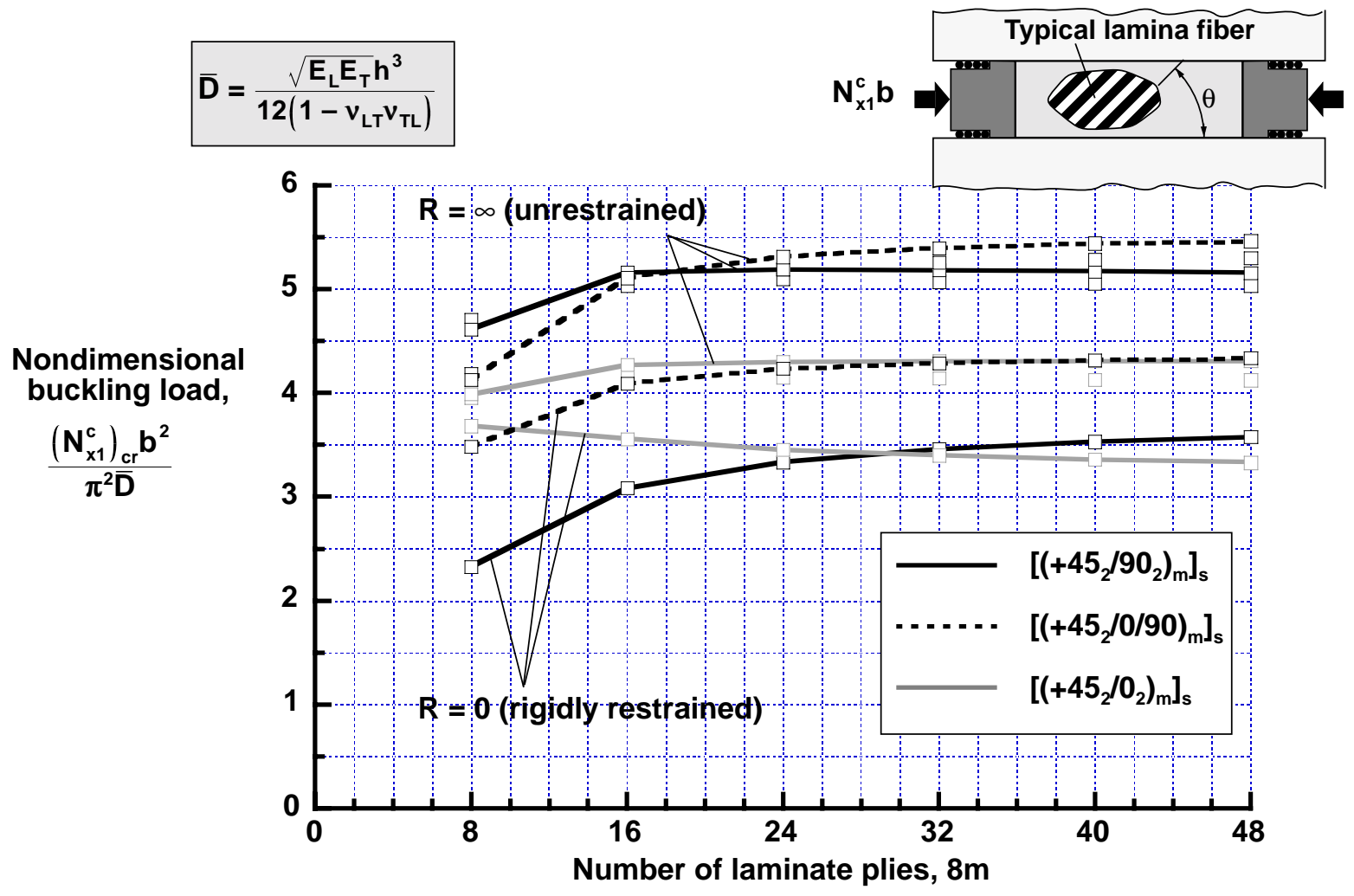

Fig. 21 Effects of compliance ratio $R$ on nondimensional buckling load for simply supported $\left[\left(+45_{2} / 0_{2}\right)_{m}\right]_{\mathrm{s}}$, $\left[\left(+45_{2} / 90_{2}\right)_{\mathrm{m}}\right]_{\mathrm{s}}$, and $\left[\left(+45_{2} / \mathbf{0 / 9 0}\right)_{\mathrm{m}}\right]_{\mathrm{s}}$ unbalanced laminates made of IM7/5260 material $\left(R_{2}=R_{3}=R\right)$.

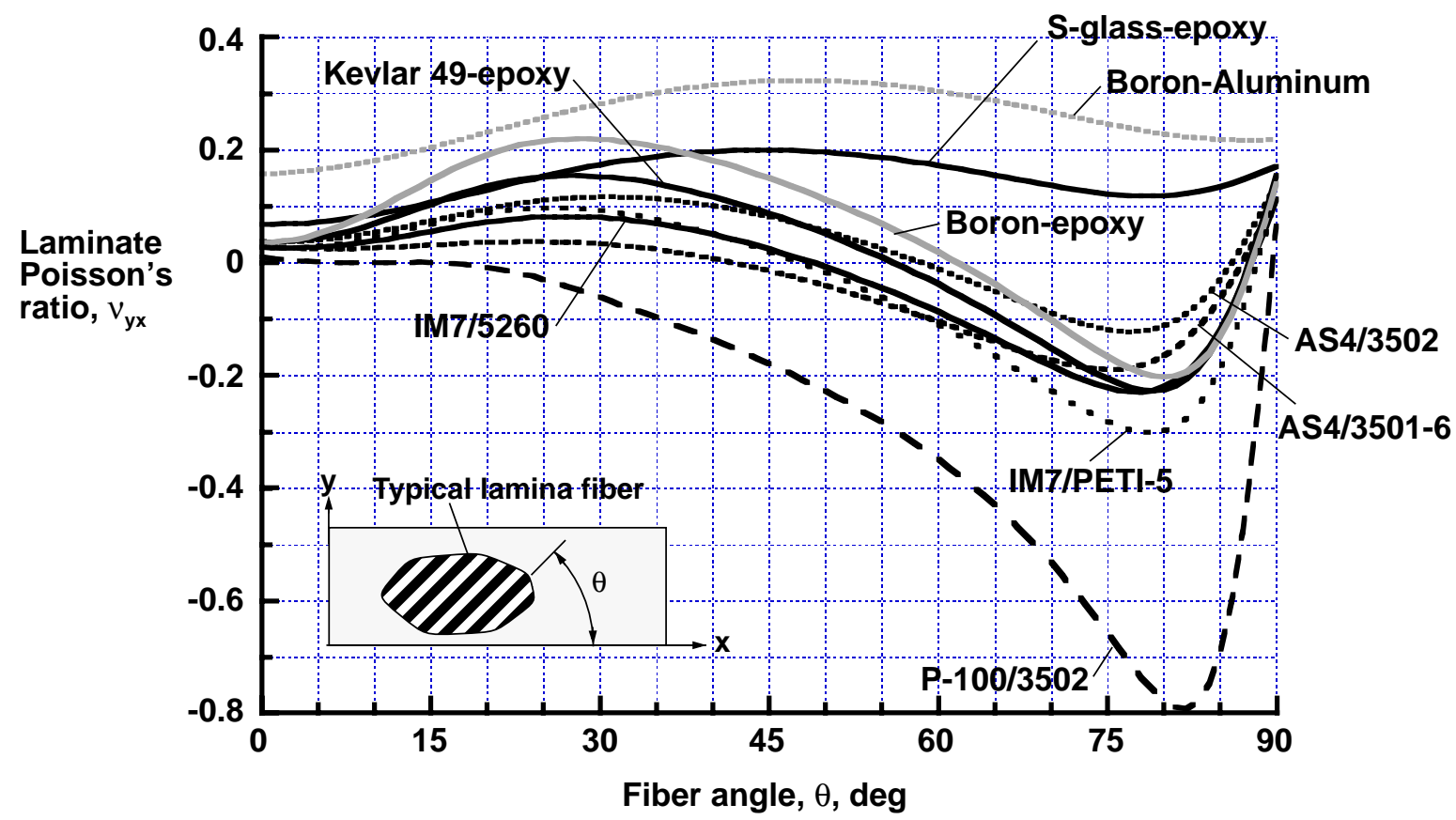

Fig. 22 Effects of lamina material properties on laminate Poisson's ratio $v_{y x}$ for $\left[+\theta_{3} / 15\right]_{s}$ unbalanced laminates. 


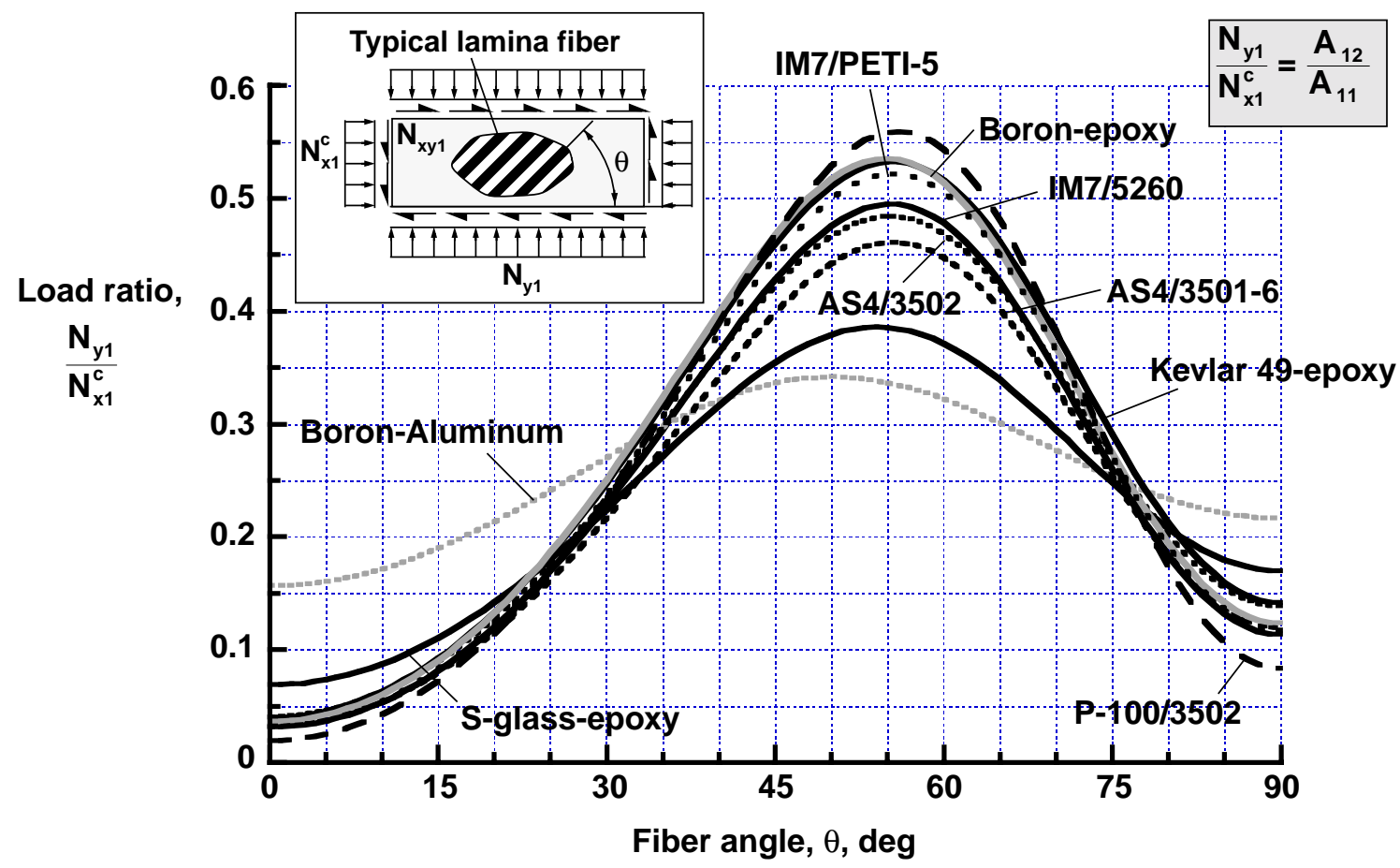

Fig. 23 Effects of lamina material properties on load ratio $N_{y 1} / N_{x 1}^{c}$ for $\left[+\theta_{3} / 15\right]_{s}$ unbalanced laminates with compliance ratios $R_{2}=R_{3}=0$ (rigidly restrained).

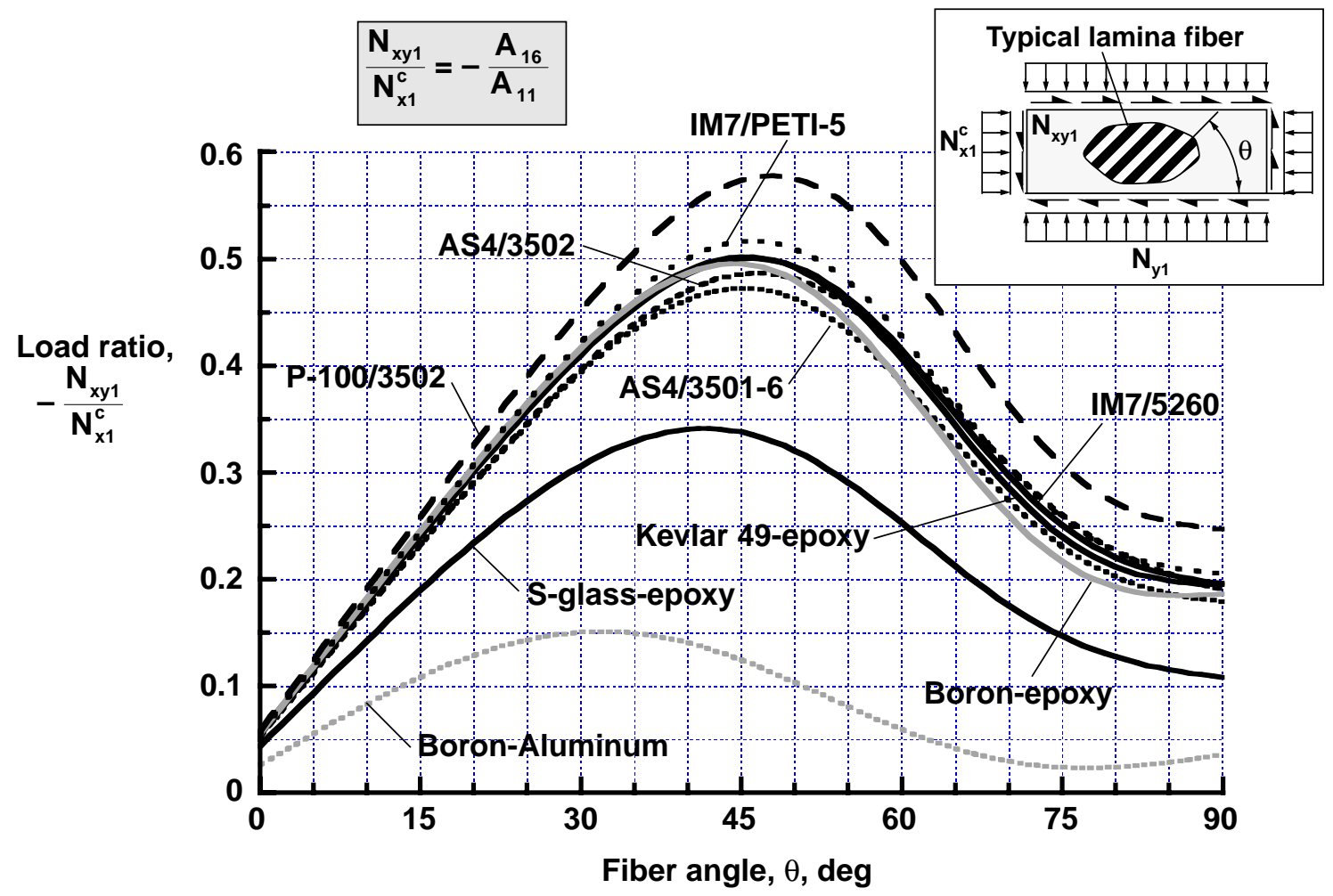

Fig. 24 Effects of lamina material properties on load ratio $N_{x y 1} / N_{x 1}^{c}$ for $\left[+\theta_{3} / 15\right]_{s}$ unbalanced laminates with compliance ratios $R_{2}=R_{3}=0$ (rigidly restrained). 


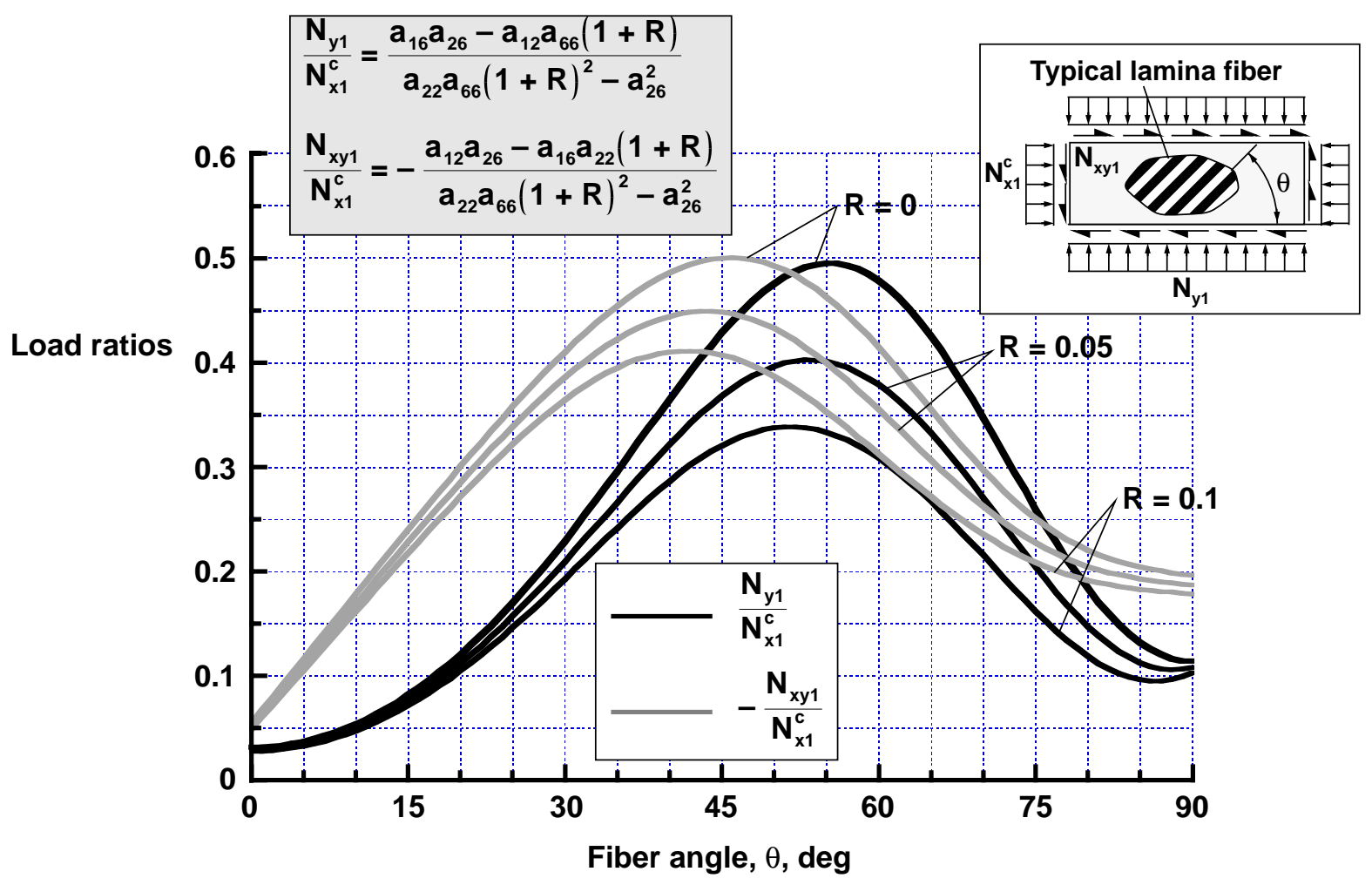

Fig. 25 Effects of compliance ratio $R$ on load ratios $N_{x y 1} / N_{x 1}^{c}$ and $N_{x y 1} / N_{x 1}^{c}$ for $\left[+\theta_{3} / 15\right]_{s}$ unbalanced laminates made of IM7/5260 material $\left(R_{2}=R_{3}=R\right)$.

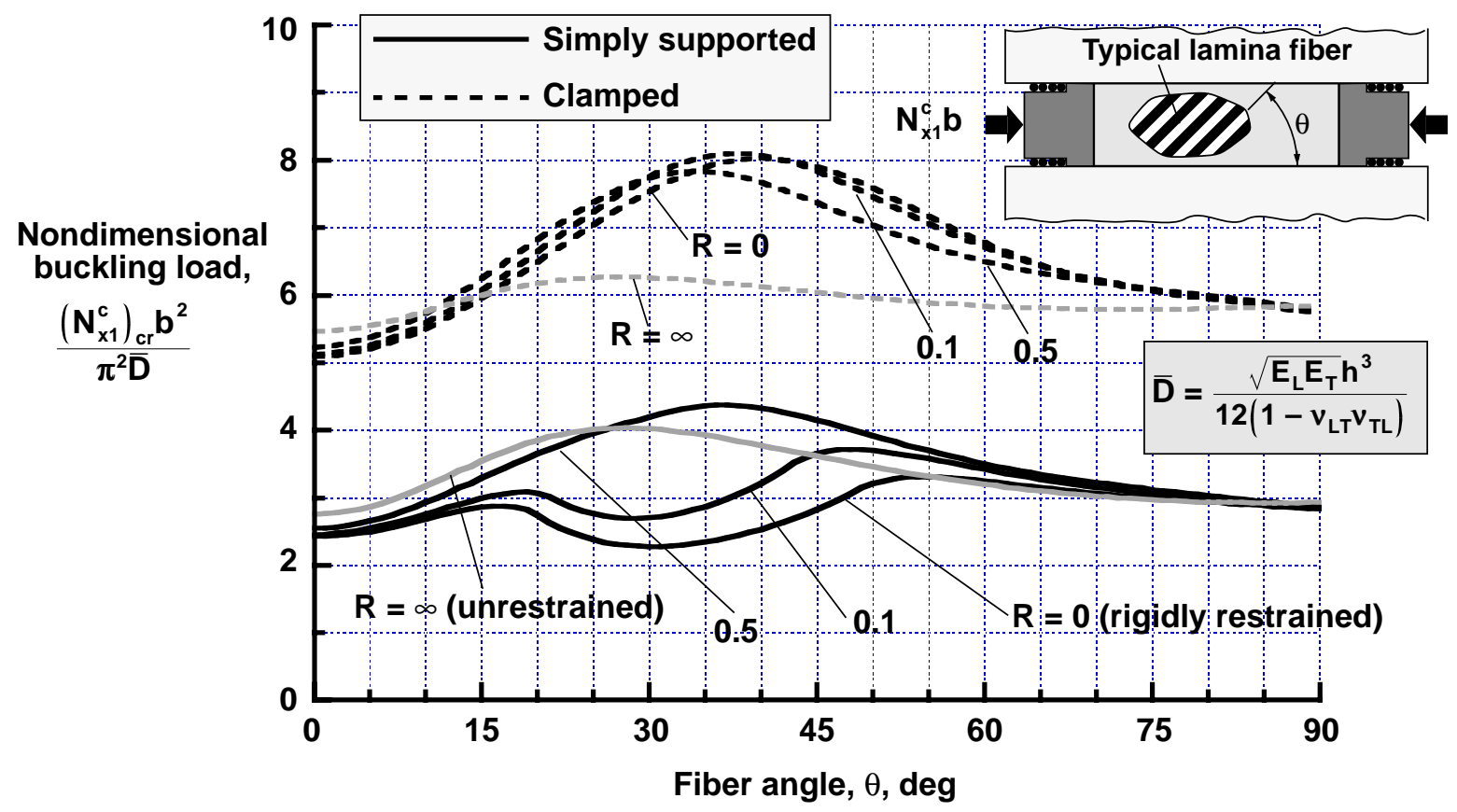

Fig. 26 Effects of compliance ratio $R$ on nondimensional buckling load for $\left[+\theta_{3} / 15\right]_{s}$ unbalanced laminates made of IM7/5260 material $\left(R_{2}=R_{3}=R\right)$. 


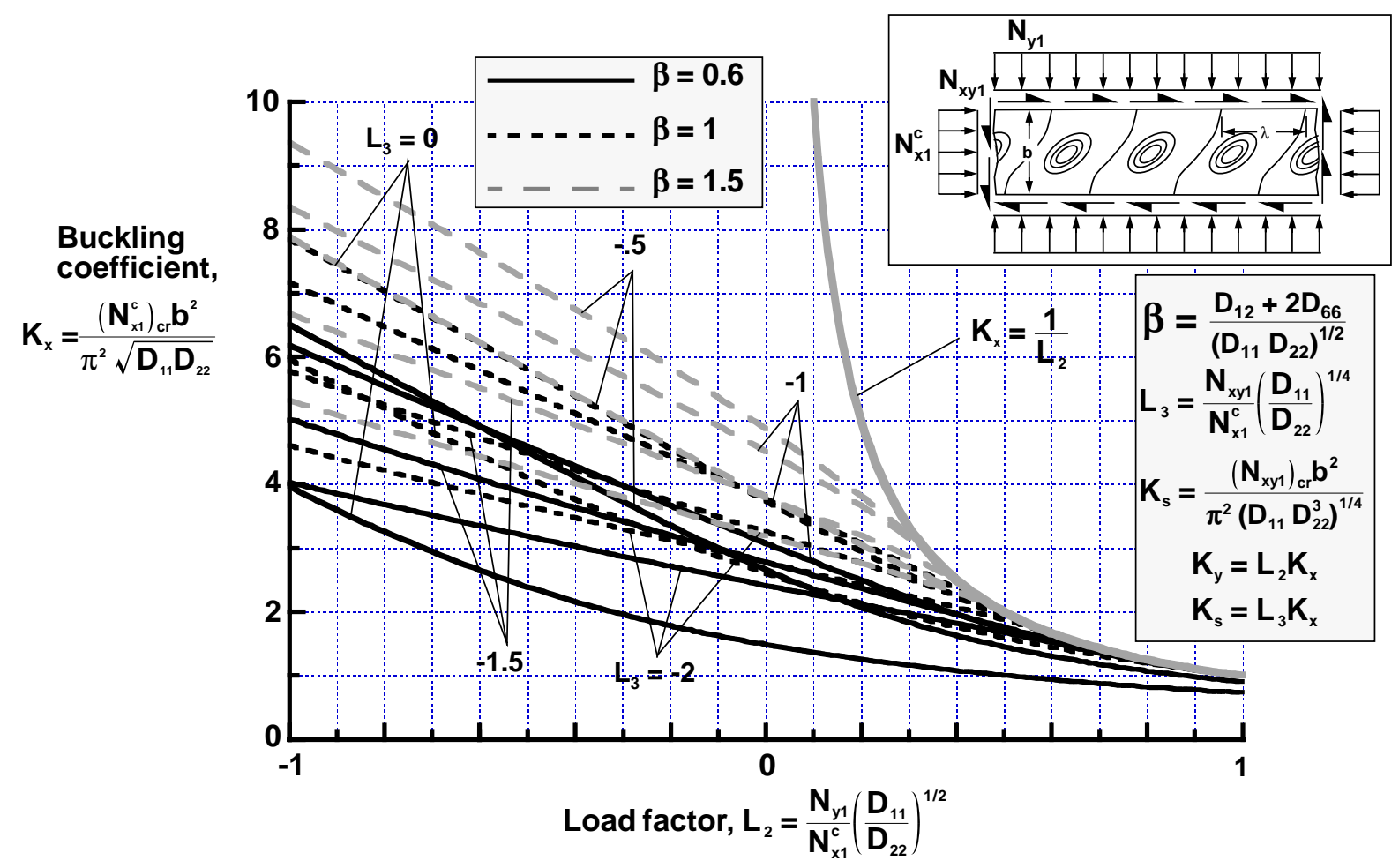

Fig. 27 Effects of orthotropy parameter $\beta$ and load factors on buckling coefficient for simply supported, specially orthotropic plates $(\gamma=\delta=\mathbf{0 . 4})$ subjected to axial compression, transverse tension or compression, and shear loads.

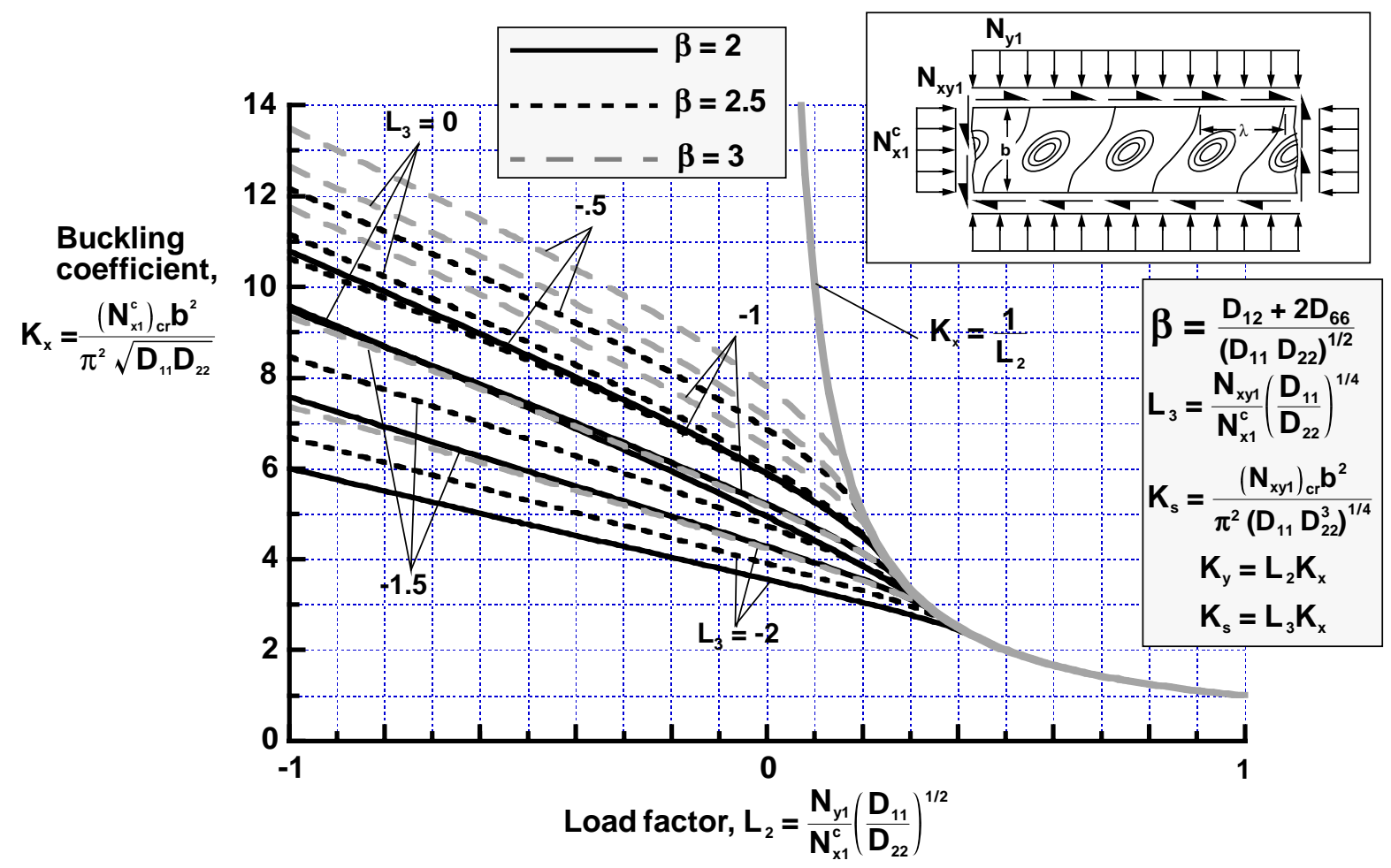

Fig. 28 Effects of orthotropy parameter $\beta$ and load factors on buckling coefficient for simply supported, specially orthotropic plates $(\gamma=\delta=\mathbf{0 . 4})$ subjected to axial compression, transverse tension or compression, and shear loads. 


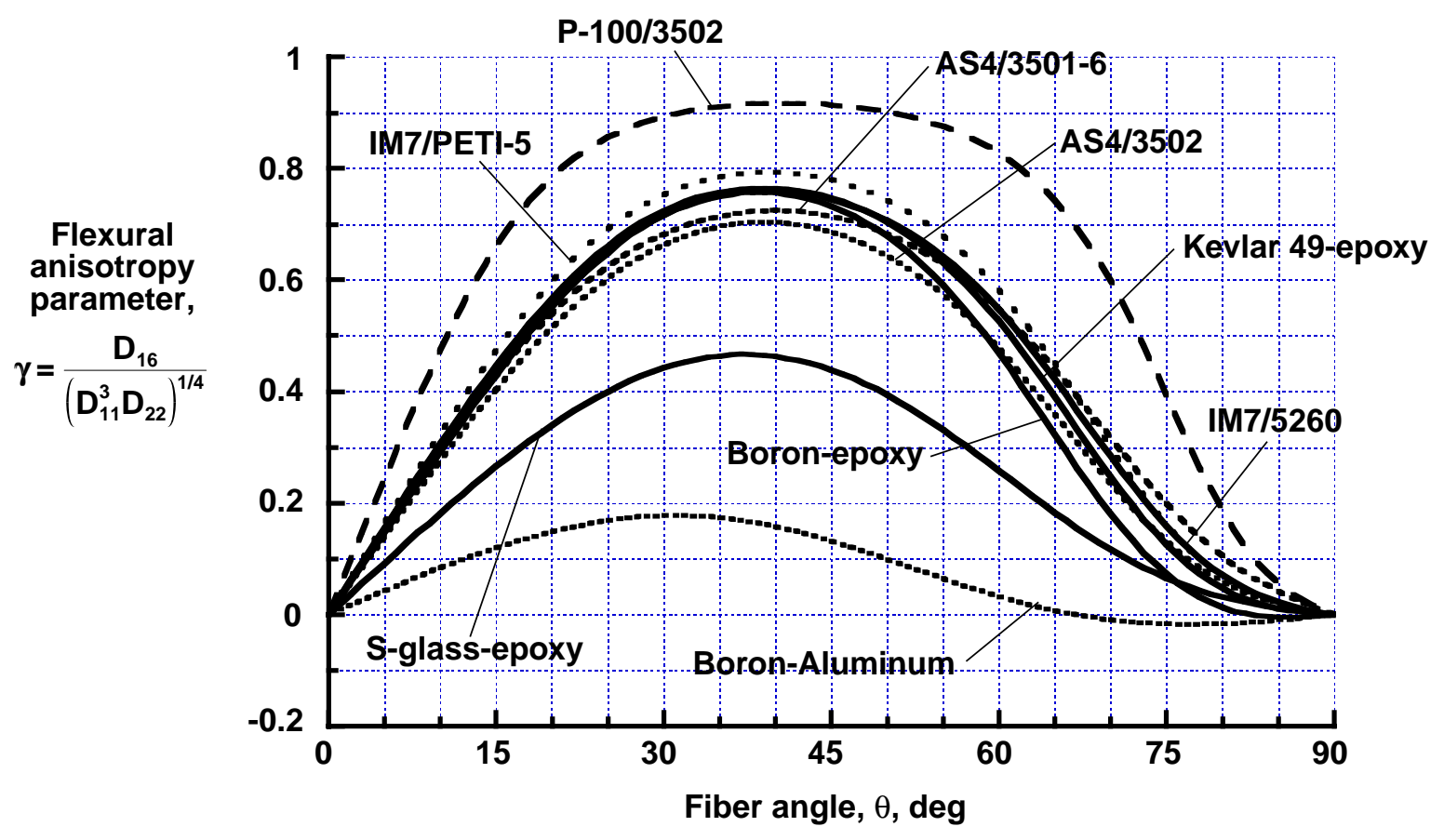

Fig. 29 Effects of lamina material properties on nondimensional flexural anisotropy parameter $\gamma$ for $[+\theta]_{m}$ unidirectional laminates $(m=1,2, \ldots)$.

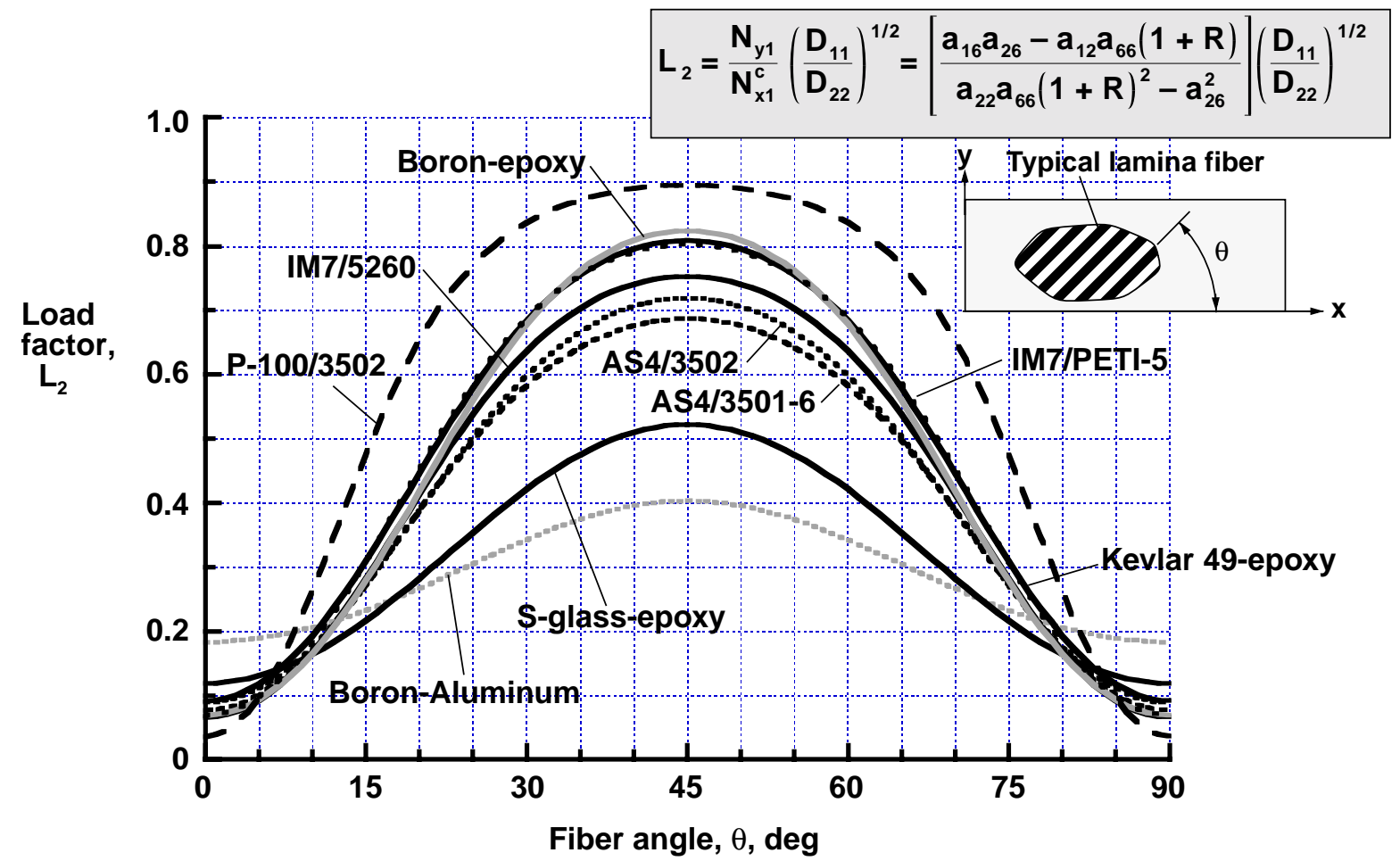

Fig. 30 Effects of lamina material properties on load factor $L_{2}$ for $[+\theta]_{m}$ unidirectional laminates $(R=0)$. 


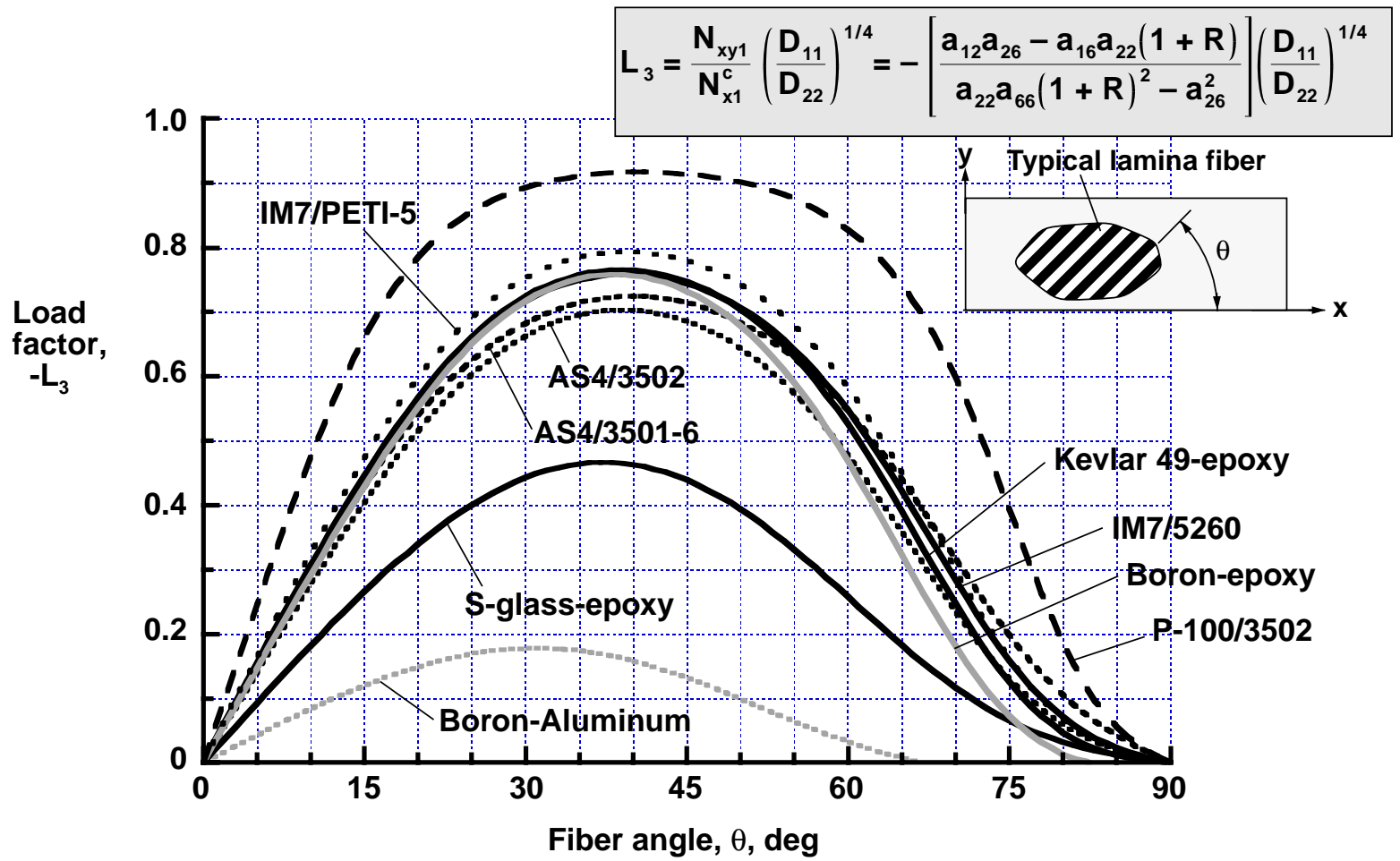

Fig. 31 Effects of lamina material properties on load factor $L_{3}$ for $[+\theta]_{m}$ unidirectional laminates $(R=0)$.

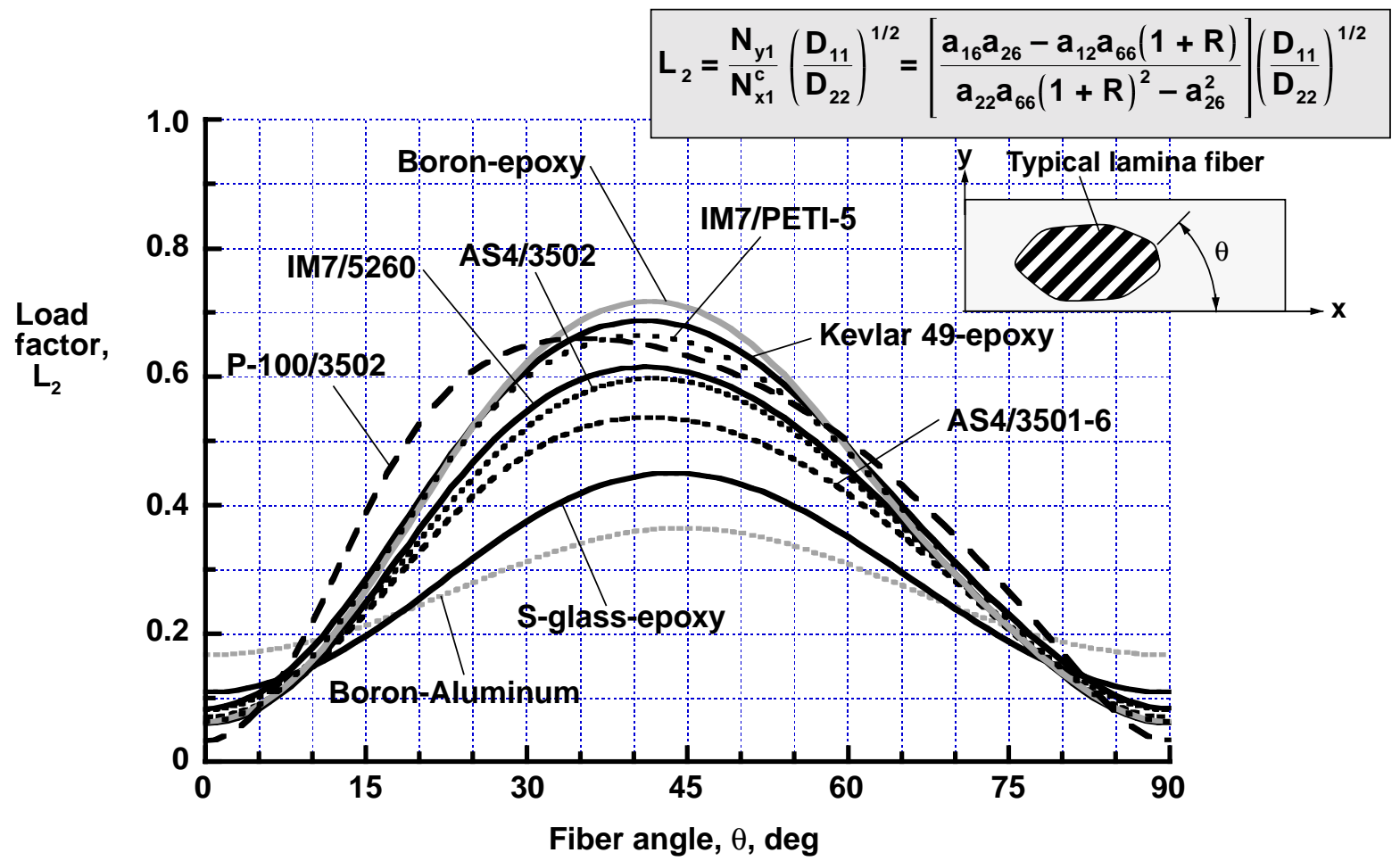

Fig. 32 Effects of lamina material properties on load factor $L_{2}$ for $[+\theta]_{m}$ unidirectional laminates $(R=0.1)$. 


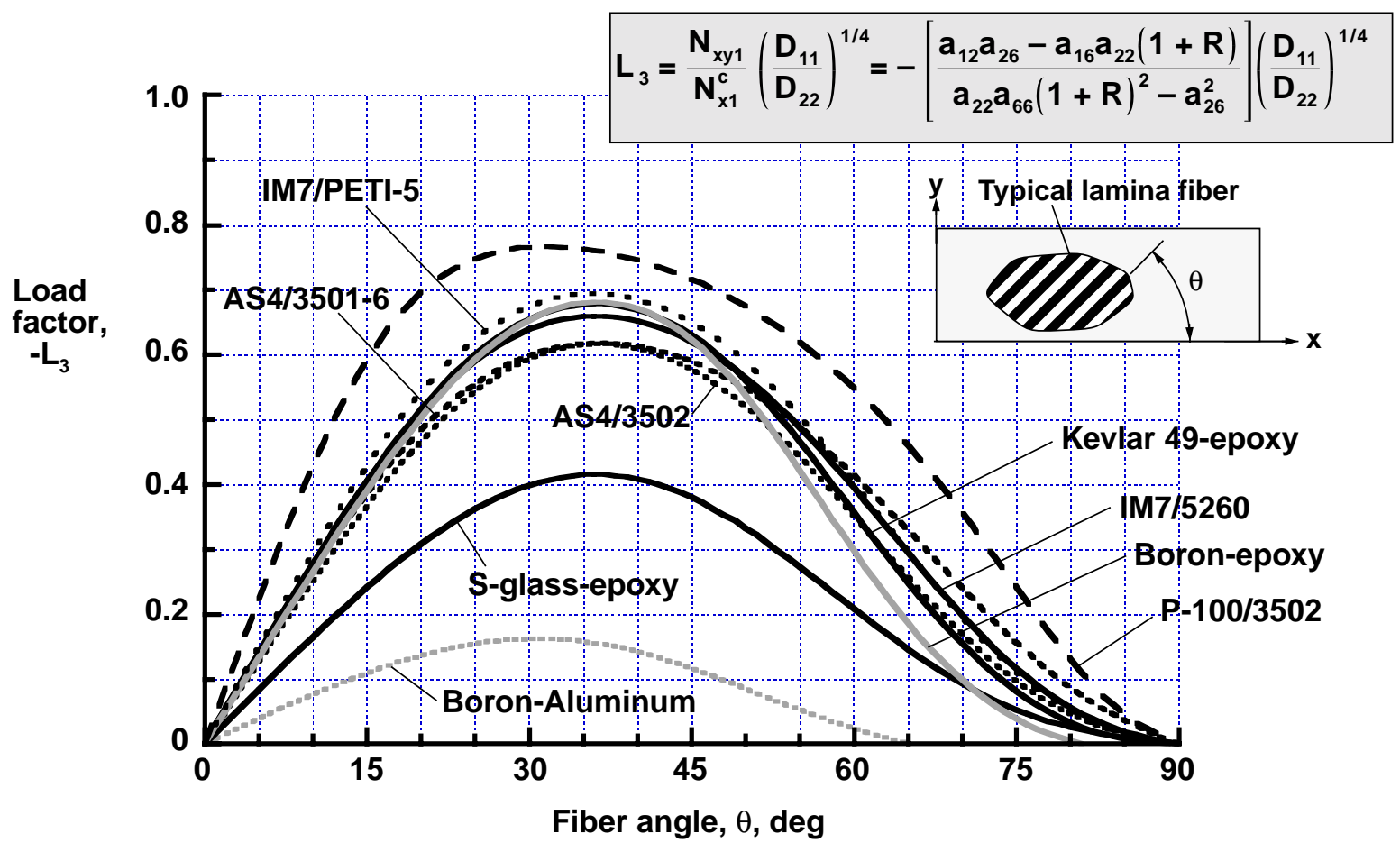

Fig. 33 Effects of lamina material properties on $\operatorname{load}$ factor $L_{3}$ for $[+\theta]_{m}$ unidirectional laminates $(R=0.1)$. 